\title{
Influence of global cosmological expansion on local dynamics and kinematics
}

\author{
Matteo Carrera* \\ Institute of Physics, University of Freiburg, Hermann-Herder-Strasse 3, D-79104 Freiburg, Germany \\ Domenico Giulin周 \\ University of Hannover, Appelstrasse 2, D-30167 Hannover, Germany周
}

(Dated: August 24, 2009)

\begin{abstract}
We review attempts to estimate the influence of global cosmological expansion on local systems. Here 'local' is taken to mean that the sizes of the considered systems are much smaller than cosmologically relevant scales. For example, such influences can affect orbital motions as well as configurations of compact objects, like black holes. We also discuss how measurements based on the exchange of electromagnetic signals of distances, velocities, etc. of moving objects are influenced. As an application we compare orders of magnitudes of such effects with the scale set by the apparently anomalous acceleration of the Pioneer 10 and 11 spacecrafts, which is $10^{-9} \mathrm{~m} / \mathrm{s}^{2}$. We find no reason to believe that the latter is of cosmological origin. However, the general problem of gaining a qualitative and quantitative understanding of how the cosmological dynamics influences local systems remains challenging, with only partial clues being so far provided by exact solutions to the field equations of General Relativity.
\end{abstract}

PACS numbers: $95.30 . \mathrm{Sf}, 04.20 . \mathrm{Cv}, 04.20 . \mathrm{Jb}, 98.80 . \mathrm{Jk}$

\section{Contents}

I. Introduction

II. Strategic outline and results

A. Improved Newtonian equations

B. Exact solutions

1. Matched solutions

2. Melted solutions

C. Kinematical effects

1. Timing and distances

2. Doppler Tracking

III. Newtonian approach

A. Restricted two-body problem in an expanding universe

B. Specifying the initial-value problem

C. Discussion of the reduced effective potential

IV. General-relativistic treatment for electromagnetically-bounded systems

A. The argument of Dicke and Peebles

B. Exact condition for non-expanding circular orbits

V. General-relativistic treatment for gravitationally-bounded systems

A. Spherically-symmetric matchings

B. The Eisenstaedt theorem

C. The Einstein-Straus vacuole revisited

D. The McVittie model

1. Interpretation of the McVittie model

2. Motion of a test particle in McVittie spacetime

3. Exact condition for non-expanding circular orbits in McVittie spacetime

\footnotetext{
*Electronic address: matteo.carrera@physik.uni-freiburg.de

$\dagger$ Electronic address: domenico.giulini@itp.uni-hannover.de

${ }^{\ddagger}$ Also at: ZARM, University of Bremen, Am Fallturm

D-28359 Bremen, Germany
}

VI. Kinematical effects

A. Einstein- versus cosmological simultaneity

B. Doppler tracking in cosmological spacetimes 26

1. Minkowski spacetime

2. General setting

3. FLRW spacetimes

4. McVittie spacetime

VII. Summary and outlook

Acknowledgments

11

A. Notation, conventions, and generalities

B. Proof of Theorem 1

C. Submanifolds

D. Spherical symmetry

1. Connection and curvature decomposition

2. Einstein equation in case of spherical symmetry

3. Misner-Sharp energy

4. Spherically symmetric perfect fluids

References

\section{INTRODUCTION}

There is by now ample evidence that our Universe is expanding on average. This means that on the largest scales one observes redshifts from structures that are interpreted as recessional motion, also called the Hubble flow. To first approximation, the relative velocity between two structures grows linearly with their mutual distance. The constant of proportionality is the so-called Hubble constant, $H_{0}$, whose value is now fairly accurately measured as being close to $70 \mathrm{Km} \cdot \mathrm{s}^{-1} \cdot \mathrm{Mpc}^{-1}$, see e.g. (Komatsu et al., 2009). This means that for any 
additional mega-parsec $\left(\mathrm{Mpc}=3.262 \times 10^{6}\right.$ lightyears $=$ $3.086 \times 10^{19} \mathrm{~km}$ ) the recessional velocity picks up an extra 70 kilometers per second. Clearly, typical peculiar velocities superimpose on the global Hubble flow. For galaxies they can be up to 1000 kilometers per second, so that the Hubble flow definitely dominates at distances above $200 \mathrm{Mpc}$, i.e. above supercluster scale. In this respect it is remarkable that Hubble's classic paper (Hubble, 1929) of 1929 plots the velocity-distance relation of extra-galactic nebulae only up to $2 \mathrm{Mpc}$, though it has to be added that in those days distances where generally underestimated, sometimes up to a factor of 10 .

For pedagogical purposes the global expansion is sometimes represented by the two-dimensional balloon model, in which three-dimensional space corresponds to the twodimensional surface of an inflating rubber balloon; see e.g. $\S 27.5$ in (Misner et al., 1973). At each point attached to the rubber material an observer sees other points attached in a state of radial recessional motion, the faster the further they are away. This picture is used to stress that each point is locally (i.e. with respect to the local rubber material) at rest but receding from all other points because space in-between is itself expanding. However, this global expansion does not affect all structures: Local overdensities in the matter distribution may inhibit space from expanding. In the balloon model of (Misner et al., 1973) this is represented by little pennies being glued onto the balloon. The rubber material underneath the coins does not expand due to the stiff glue which holds it in place. The question arises what, in reality, are the structures corresponding to the coin and what dynamical mechanism provides the glue? It is often heard that 'bound systems' do not participate in the global expansion, or that systems below the scale of galaxy clusters 'break away' from the Hubble flow. But what does 'bound' and 'break away' really mean? ${ }^{1}$ For example, is it obvious that the Astronomical Unit is not affected by global expansion (compare (Krasinsky and Brumberg, 2004; Standish, 2004)) or can it even be, as e.g. suggested in (Fahr and Siewert, 2008), that our Universe is contracting on small scales while it expands in the large? If so, what precisely would rule the relation between contracting and expanding scales?

The purpose of this paper is to review and discuss attempts that aim to make precise and answer some of these fundamental questions, taking due account of the dynamical laws and the kinematical framework of General Relativity. We will emphasize the changes in kinematical relations within time-dependent spacetime geometries, which seem to be widely neglected in related discussions.

Next to being a question of fundamental interest, the raised issue also needs to be clarified quantitatively in connection with more practical

\footnotetext{
${ }^{1}$ For a recent discussion on the meaning of 'joining the Hubble flow' see (Barnes et al., 2006).
}

aims, like, e.g., the modeling of celestial reference frames (Klioner and Soffel, 2005). The specific question of whether the global expansion has any influence on the local dynamics and kinematics within the Solar System has recently also attracted increasing attention in connection with the so-called 'PioneerAnomaly' Anderson et al., 1998; Anderson et al., 2002; Markwardt, 2002; Nieto and Turyshev, 2004; Turyshev et al., 2005a, b), henceforth abbreviated by PA. Here frequency-measurements in Doppler tracking are translated into standard kinematical quantities, like velocity and acceleration. The result shows an anomalous acceleration of the Pioneer satellites directed towards the center of the Solar System. In Markwardt, 2002) the magnitude of this acceleration is reported to be $a=8.6 \pm 1.34 \times 10^{-10} \mathrm{~m} \cdot \mathrm{s}^{-2}$. Note that such an apparently small acceleration amounts to variations in spatial localization of nearly 500 kilometers after 10 years. It so happens that the magnitude of this acceleration is very close to the product of the current value of the Hubble constant, $H_{0}$, and the velocity of light in vacuum:

$$
\begin{aligned}
H_{0} c & \approx\left(70 \mathrm{~km} \cdot \mathrm{s}^{-1} \cdot \mathrm{Mpc}^{-1}\right)\left(3 \times 10^{5} \mathrm{~km} \cdot \mathrm{s}^{-1}\right) \\
& =7 \times 10^{-10} \mathrm{~m} \cdot \mathrm{s}^{-2} .
\end{aligned}
$$

Whether this 'almost coincidence' of numbers does indeed have any deeper significance can and should only be decided on the basis of reliable estimates within the dynamical framework of General Relativity. There already exist various speculations and claims in the literature that try to attribute the PA to either simple kinematical (e.g. (Rosales and Sanchez-Gomez, 1998)) or dynamical (e.g. (Fahr and Siewert, 2008)) effects of a time varying background geometry, though none of them does justice to the requirements posed by General Relativity. ${ }^{2}$ This is clearly a very difficult task: There is very little analytical knowledge of how to model in terms of exact solutions, or at least in terms of controlled approximations to exact solutions, the hierarchy of mutually embedded systems: Solar System $\rightarrow$ Galaxy $\rightarrow$ Local Group $\rightarrow$ Cluster $\rightarrow$ Supercluster $\rightarrow$ Standard-Cosmological Solution. Usually we expect each such system to define a typical length scale beyond which we may consider it as quasi isolated (Cox, 2007). But, clearly, whether this is a valid assumption or not can only be decided on the basis of a self-consistent dynamical consideration. In our context all this suggests to first study the influence of cosmic expansion on the most simple systems immersed in an otherwise homogeneous cosmological background. We will see that this already poses a number of non-trivial analytical as well as conceptual problems.

In this article we will derive upper bounds for various effects of global expansion on local systems in the con-

\footnotetext{
2 The reader will soon find out that we disagree with all such claims.
} 
text of such simple models. The idea here is that the upper bounds so derived will a fortiori be upper bounds in more realistic models, since a further embedding of the system we consider into a higher structure of local overdensities will further suppress the influence of cosmological expansion. This is evidently true in situations in which the spherically symmetric Einstein-Straus model applies, but can also be argued for as a result of taking into account small-scale anisotropies in the matter distribution, as has been done from first-order perturbations of the Newtonian equations (Dominguez and Gaite, 2001). We conclude form this that if we find the relevant upper bounds to be outside current experimental reach, this will maintain to be the case in more realistic contexts.

\section{STRATEGIC OUTLINE AND RESULTS}

\section{A. Improved Newtonian equations}

The strategies that so far have been followed are twofold: Either one studies modified Newtonian or special relativistic equations of motions for two pointparticles with a force of mutual attraction (gravitational or electromagnetic). The modifications are derived from putting the system into a fixed standardcosmological background (usually spatially flat) without back-reactions being taken into account. We shall discuss this approach in Sections [III] and [V] Our discussion, based on (Carrera and Giulini, 2005), complements the perturbative analysis in (Cooperstock et al., 1998) which misses all orbits which are unstable under cosmological expansion (which do exist). In this respect we follow a very similar strategy as, e.g., in the more recent papers by (Price, 2005) (the basic idea of which goes back at least to (Pachner, 1963, 1964) ) and also (Adkins et al., 2007), though we think that there are also useful differences. We also supply quantitative estimates and clarify that the improved Newtonian equations of motion are written in terms of the right coordinates (non-rotating and metrically normalized). The purpose of this model is to develop a good physical intuition for the qualitative as well as quantitative features of any dynamical effects involved.

Eventually the Newtonian model just mentioned has to be understood as a limiting case of a genuinely relativistic treatment. For the gravitational case this is done in Section $\mathrm{V}$ (an alternative and more geometric derivation is given in Section VI.B), where we employ the McVittie metric to model a spherically symmetric mass embedded in a spatially flat Friedmann-LemaitreRobertson-Walker (FLRW) universe. The geodesic equation is then, in a suitable limit, shown to lead to the improved Newtonian model discussed above (see also (Carrera and Giulini, 2005)). The same holds for the electromagnetic case, as we show in Section IV. There we take a slight detour to also reconsider a classic argument by Dicke \& Peebles (Dicke and Peebles, 1964), which allegedly shows the absence of any relevant dynamical effect of global expansion. Its original form only involved the dynamical action principle together with some simple scaling argument. Since this reference is one of the most frequently cited in this field, and since the simplicity of the argument (which hardly involves any real analysis) is definitely deceptive, we give an independent treatment that makes no use of any hypothetical scaling rules for physical quantities other than spatial lengths and times. Our treatment, which follows (Carrera and Giulini, 2005), also reveals that the original argument by Dicke \& Peebles is insufficient to discuss leading order effects of cosmological expansion. It is therefore also ineffective in its attempt to contradict (Pachner, 1963, 1964).

\section{B. Exact solutions}

The other approach consists of finding exact solutions to Einstein's field equations for an inhomogeneous situation that, in the most simple case, models a single, quasilocalized, non-rotating, electrically neutral inhomogeneity within a FLRW universe. Using this inhomogeneous solution as background one can then study the motion of test particles (following geodesics in the background geometry) and, in particular, the influence of expansion on this motion.

This approach can be subdivided into two strategies. The first tries to literally construct a new exact solution out of two known ones, so that the new solution contains a connected piece from each of the two old ones as isometric submanifolds. These we refer to as matched solutions. This is relaxed in the second, more general strategy, where the new solution is merely required to somehow approximate the relevant part of each of the two old solutions in some region. These we refer to as melted solutions. Needless to say that melted solutions offer a much greater variety for construction than matched ones. However, it is also true that often not much is known about the proper physical interpretation of the former. In this respect the matching solutions usually provide a much clearer picture.

According to the above requirements, in both cases we shall restrict attention to spherically symmetric spacetimes which, loosely speaking, approximate a FLRW solution of standard cosmology for 'large radii' and a non-charged, non-rotating compact object characterized by the exterior Schwarzschild solution for 'small radii'. (Clearly there must be some characteristic radius in terms of which 'large' and 'small' radii are defined.) Also, one often restricts attention to the spatially flat FLRW models for simplicity, which also seems justified in view of current cosmological data which are compatible with spatial flatness. 


\section{Matched solutions}

A first approach to the matching idea was initiated by Einstein and Straus (Einstein and Straus, 1945, 1946) in 1945 and later worked out in more analytical detail by Schücking (Schücking, 1954). Here the matched solution is really such that for radii smaller than a certain matching radius, $R_{v}$ (henceforth called the vacuole or Schücking radius), it is exactly given by the Schwarzschild solution (exterior for a black hole, exterior plus interior for a star) and for radii above this radius it is exactly given by a FLRW universe for dust matter without cosmological constant (this can be generalized, see below). The radius $R_{v}$ is a function of the central gravitational mass $M$ and the cosmological mass-density $\varrho$, through the latter of which it also depends on the cosmological time $t$. It is determined by

$$
\frac{4 \pi}{3} R_{v}^{3} \cdot \varrho=M .
$$

This formula holds for flat as well as curved FLRW models if 'radius' is taken to mean 'areal radius', the definition of which is that a two-sphere of areal radius $R$ has a proper surface area of $4 \pi R^{2}$. In flat space the areal radius coincides with the proper radius (the geodesic distance between the center and any point on the sphere), so that $\frac{4 \pi}{3} R_{v}^{3}$ is just the proper volume inside the sphere of radius $R_{v}$ (cf. Section V.A ). However, in backgrounds of positive (negative) curvature this expression is smaller (larger) than the proper volume (the proper volume grows faster (slower) with areal radius) and hence, for given $\varrho$, the left-hand side of (2) is also smaller (larger) than the proper mass of the dust contained within a sphere of areal radius $R_{v}$.

Here we recall that the gravitational mass of a lump of matter is not just proportional to the amount of matter (baryons) in that region. For example, the kinetic energy as well as the gravitational binding energy also contribute to the gravitational mass. This is expressed in formula (D47) of Appendix D.4, where further explanations will be provided. As is well known, the mathematical characterization of appropriate notions of quasi-local gravitational mass that would apply to general spacetimes is a notoriously difficult problem to which various attempts for solutions exist; see (Szabados, 2004) for the current status. However, in the spherically-symmetric case, to which we restrict attention, the so-called Misner-Sharp energy gives a satisfying and convenient concept of active gravitational mass. Its definition will be given in Section V.A and more details, including its equality in value to the Hawking mass, are discussed in the Appendices D.3 and D.4.

The original construction by Einstein and Straus and its analytical completion by Schücking were quite complicated. We will give a much simpler and conceptually clearer description in Section V.C, using a suitable reformulation of the condition for the matching of solutions. However, it is not hard to gain some intuitive understanding for the matching construction and the value of
$R_{v}$ as defined by (2). Let us for the moment restrict to the spatially flat case and consider the homogeneous and isotropic dust-filled universe at some moment of time $t$. The dust within a 3-ball of proper radius $R_{v}$ represents an amount of matter of total mass $M$ as given by (2). Now compress this amount of matter in a spherically symmetric fashion until it becomes a compact star or a black hole. In Newtonian gravity the gravitational field outside a spherically symmetric mass distribution only depends on the total mass and not on its radial density distribution. This is also true in General Relativity, which is essentially the content of Birkhoff's theorem. ${ }^{3}$ Hence the above compression preserves equilibrium (albeit an unstable one, see below) for the dust particles just outside the boundary-sphere of radius $R_{v}$. For radii smaller than $R_{v}$ we have the Schwarzschild solution (which is the unique non-trivial spherically symmetric vacuum solution according to Birkhoff's theorem) which therefore matches to the FLRW solution for $R \geq R_{v}$ at the boundary $R=R_{v}$ where the matter density is discontinuous. The spatial two-sphere $R=R_{v}$ is comoving with the Hubble flow, meaning that its proper surface area grows in case of expansion. Finally, in case of constant positive (negative) spatial curvature, (2) tells us that the matched Schwarzschild solution has a smaller (larger) mass than the mass that the amount of dust represents within the ball of areal radius $R_{v}$ within the FLRW universe.

The Einstein-Straus model can be generalized in several ways. Instead of cutting out one ball, one can cut several non-overlapping ones and fill in the interiors with Schwarzschild geometries of appropriate masses. For obvious reasons these are sometimes referred to as 'Swiss-Cheese models'. These, in turn, can be generalized to the cases of non-vanishing cosmological constant (Balbinot et al., 1988) or non-vanishing pressure (Bona and Stela, 1987). Finally, the EinsteinStraus model can be generalized to spherically symmetric but inhomogeneous Lemaître-Tolman-Bondi (LTB) cosmological backgrounds (Bonnor, 2000).

Since for the Einstein-Straus model the geometry within $R \leq R_{v}$ is exactly Schwarzschild (for vanishing cosmological constant) or Schwarzschild-de Sitter spacetime (for non-vanishing cosmological constant), it is clear that any dynamical system situated in this background geometry (no back reaction) only detects that part of the cosmic expansion that is due to a non-vanishing cosmological constant. In particular, for vanishing cosmological constant, the cosmic expansion that goes on outside the expanding vacuole $R=R_{v}$ is not felt from within. Hence global expansion due to ordinary (localizable) matter can, in principle, be completely inhibited by local inhomogeneities.

There are, however, several severe problems concern-

\footnotetext{
${ }^{3}$ An elegant proof of Birkhoff's theorem will appear as a byproduct from our considerations in Appendix D.3
} 
ing the Einstein-Straus approach. First of all, it cannot provide a realistic model for the environment of small structures in our Universe, 'small' meaning below the scales of galaxy clusters or superclusters. To see this, apply (2) to a spatially flat universe whose background matter density $\varrho$ is given by the critical density

$$
\varrho_{\text {crit }}:=\frac{3 H_{0}^{2}}{8 \pi G},
$$

where $G$ is Newton's constant. Then (2) gives

$$
R_{v}=\left(R_{S} R_{H}^{2}\right)^{1 / 3} \approx\left(\frac{M}{M_{\odot}}\right)^{1 / 3} 400 \mathrm{ly}
$$

where

$$
\begin{aligned}
& R_{S}:=\frac{2 G M}{c^{2}} \approx \frac{M}{M_{\odot}} 3 \mathrm{~km}, \\
& R_{H}:=\frac{c}{H_{0}} \approx 4 \mathrm{Gpc} \approx 1.3 \times 10^{23} \mathrm{~km},
\end{aligned}
$$

are the Schwarzschild radius for the mass $M$ and the Hubble radius, respectively. $M_{\odot}=2 \times 10^{30} \mathrm{~kg}$ is the solar mass.

For a single solar mass this gives a vacuole radius of almost 400 lightyears, which is almost two orders of magnitude larger than the average distance of stars in our Galaxy. Therefore, the Swiss-Cheese model cannot apply at the scale of stars in galaxies. This changes as one goes to larger scales. For example, the Virgo cluster is estimated to have a mass of approximately $10^{15}$ solar masses (Fouqué et al., 2001) ${ }^{4}$, which makes its vacuole radius $10^{5}$ times larger than that for a single solar mass, so that it is approximately given by $10 \mathrm{Mpc}$. This is just a little smaller than the average distance of groups and clusters of galaxies within the Virgo supercluster. Hence the Einstein-Straus approach might well give viable models above cluster scales. Similar conclusion can be drawn for the vacuole construction in LTB spacetimes (Bonnor, 2000): There it is argued that the vacuole might be as big as the Local Group.

The Einstein-Straus solution (as well as its generalization for LTB spacetimes given by Bonnor) may also be criticized on theoretical grounds. An obvious one is its dynamical instability: slight perturbations of the matching radius to larger radii will let it increase without bound, slight perturbations to smaller radii will let it collapse. This can be proven formally (e.g. (Krasiński, 1998), Ch. 3 and (Bonnor, 2000)) but it is also rather obvious, since $R_{v}$ is defined by the equal and opposite gravitational pull of the central mass on one side and the cosmological masses on the other. Both pulls increase as one moves towards their side, so that the equilibrium position must correspond to a local maximum

\footnotetext{
4 Their considerations are based on a LTB model for the cluster.
}

of the gravitational potential. Another criticism of the Einstein-Straus solution concerns the severe restrictions under which it may be generalized to non sphericallysymmetric situations; see e.g. (Mena et al., 2002, 2003, 2005; Senovilla and Vera, 1997).

\section{Melted solutions}

The above discussion shows that the Einstein-Straus approach does not give us useful information regarding the dynamical impact of cosmic expansion on structures well below the scales of galaxy clusters. For this reason other exact solutions are sought. In this respect we wish to remind the reader on the following general aspect: In physics we are hardly ever in the position to mathematically rigorously model physically realistic scenarios. Usually we are at best either able to provide approximate solutions for realistic models or exact solutions for approximate models, and in most cases approximations are made on both sides. The art of physics then precisely consists in finding the right mixture in each given case. However, in this process our intuition usually strongly rests on the existence of at least some 'nearby' exact solutions. Accordingly, one seeks exact solutions in General Relativity that, with some degree of physical approximation, model a spherically symmetric body immersed in an expanding universe. However, it is not as easy as one might think at first to characterize 'body' and 'immersed'. ${ }^{5}$ Clearly it is associated with some inhomogeneity in form of a spatial region with an overdense matter distribution, as compared to that of the approximately homogeneous distribution far out. But a body should also be quasi-isolated in order to be distinguishable form a mere local density fluctuation with smooth transition. Typical exact solutions that models the latter are the LTB solutions, in which matter is represented by pressureless dust that freely falls into the local overdense inhomogeneity. In some sense, these form the other extreme to the Einstein-Straus solutions in that they make the transition as smooth and mild as one wishes. Here we shall be interested in models that somewhat lie inbetween these extremes.

An attempt to combine an interior Schwarzschild solution (representing a star) and a flat FLRW universe was made by Gautreau (Gautreau, 1984). Here the matter model consists of two components, a perfect fluid with pressure and equation of state $p=p(\varrho)$ outside the star, and the superposition of this with the star's dust-matter inside the star. However, Gautreau also made the assumption that the matter outside the

\footnotetext{
${ }^{5}$ In a linear theory, the 'simultaneous presence' of two structures, like a local inhomogeneity in an 'otherwise' homogeneous background, naturally corresponds to the mathematical operation of addition of the corresponding individual solutions. In a nonlinear theory, however, no such simple recipe exists.
} 
star moves on radially infalling geodesics, which is only consistent if the pressure outside is spatially constant. Thus one is reduced to exact FLRW outside the star (van den Bergh and Wils, 1984) or the LTB model. (Further remarks may be found in (Krasiński, 1998), e.g. p. 113 and 165.) Other solutions, modeling a black hole in a cosmological spacetime, have been given in the literature. However, these solutions model objects which are either rotating (Ramachandra et al., 2003; Vaidva, 1977, 1984), charged (Gao and Zhang, 2004), or both (Patel and Trivedi, 1982). Surveys on the subject of cosmological black holes are Vishveshwara, 2000) and (McClure, 2006). Further interesting solutions are given in (Raiesh Navak et al., 2001) and in (Faraoni and Jacques, 2007; Sultana and Dver, 2005). The solutions proposed in the latter two works can be seen as generalizations of McVittie's model (McVittie, 1933), which we extensively discuss in Section V.D. A crucial feature of these solutions is, however, that the strength of the inhomogeneity ${ }^{6}$ varies in time, whereas for the McVittie model it remains constant. These solutions are of interest in their own right (for a detailed analysis see (Carrera and Giulini, 2009)), but our goal here is to focus on the effects due to cosmological expansion and not on the effects due to a changing strength of the central inhomogeneity. The solution proposed in the former work (Rajesh Navak et al., 2001) is the melting of a Schwarzschild spacetime in an Einstein's static universe. This is a purely static solution whose properties and geodesics where studied in (Ramachandra and Vishveshwara, 2002). For our purposes, however, this spacetime is not interesting since it is asymptotically an Einstein universe, and hence not in agreement with the present picture of our Universe at large scales.

For these reasons in Section V.D we shall pay special attention to the McVittie model. This contains a distinguished central object in the sense that the mass within a sphere centered at the inhomogeneity splits into a piece that comes from the continuously distributed cosmological fluid (with pressure) and a constant piece that does not depend on the radius of the enclosing sphere; see our Eq. (82). Moreover, the latter piece is also constant in time, meaning that the strength of the central inhomogeneity remains constant. By the way, McVittie's solutions contain the Schwarzschild-de Sitter one as a special case, which was recently used in the literature to estimate the effects of cosmological expansion on local systems (Hackmann and Lämmerzahl, 2008c; Kagramanova et al., 2006). In Section V.D.2 we show that in a suitable weak-field and slow-motion approximation the geodesic equation in $\mathrm{McVittie}$ spacetime reduces to the improved Newtonian equations discussed earlier.

\footnotetext{
${ }^{6}$ In Section V.D.1 we will identify the strength of the inhomogeneity with the Weyl part of the Misner-Sharp energy.
}

An alternative and more geometric derivation of the improved Newtonian equations for the McVittie case is presented in Section VI.B (see Eq. (149)).

\section{Kinematical effects}

\section{Timing and distances}

Neither the improved Newtonian model nor other general dynamical arguments make any statement about possible kinematical effects, i.e. effects in connection with measurements of spatial distances and time durations in a cosmological environment whose geometry changes with time. This is an important issue if one wants to perform the tracking of a spacecraft, that is a 'mapping out' of its trajectory, which basically means to determine its simultaneous spatial distance to the observer at given observer times. But we know from General Relativity that the concepts of 'simultaneity' and 'spatial distance' are not uniquely defined. This fact needs to be taken due care of when analytical expressions for trajectories, e.g. solutions to the equations of motion in some arbitrarily chosen coordinate system, are compared with experimental findings. In those situations it is likely that different kinematical notions of simultaneity and distance are involved which need to be properly transformed into each other before being compared. For example, these transformations can result in additional acceleration terms involving the product (1). Accordingly, there were claims in the literature that these kinematical effects could account for the PA; see e.g. (Nieto et al., 2005; Nottale, 2003; Palle, 2005; Ranada, 2005; Rosales and Sanchez-Gomez, 1998; Rosales, 2002) and also statements to the contrary (Lämmerzahl et al., 2006). In Section VI.A. following (Carrera and Giulini, 2005), we will confirm the existence of kinematical acceleration terms proportional to $H_{0} c$, but they are suppressed with additional powers of $\beta=v / c$, which renders them irrelevant as far as the PA is concerned.

\section{Doppler Tracking}

The discussion in Section VI.B is based on Carrera and Giulini, 2006). We explain in some detail the geometric theory for setting up the kinematical framework in which Doppler tracking should be discussed in order to properly speak of relative velocities and accelerations. This is a non-trivial issue which is, in our opinion, not properly appreciated in the literature on this subject (related general discussions are (Bini et al., 1995; Bolós, 2007)). Using this setting, we show how to derive an exact Doppler-tracking formula for a flat FLRW universe. This we use to give reliable upper bounds for kinematical effects caused by cosmic expansion. We also discuss generalizations to McVittie spacetime. Even though such effects exist, they again turn out to be irrelevant for the PA. 


\section{NEWTONIAN APPROACH}

In order to gain intuition we consider a simple bounded system, say an atom or a planetary system, immersed in an expanding cosmos. We ask for the effects of this expansion on our local system. Does our system expand with the cosmos? Does it expand only partially? Or does it not expand at all? Here we shall not be concerned with the far more complex problem of how stable large-scale structures may emerge from unstable local gravitational dynamics in an expanding universe. This has been discussed in (Buchert and Dominguez, 2005) and references therein.

\section{A. Restricted two-body problem in an expanding universe}

We consider the dynamical problem of two bodies attracting each other via a force with $1 / R^{2}$ fall-off. For simplicity we may think of one mass as being much smaller than the other one, though this is really inessential. One may think of two galaxies, a star and a planet, a planet and a spacecraft, or a (classical) atom given by an electron orbiting around a proton. The system is placed into an isotropically expanding ambient universe. We wish to know the leading order influence of the ambient expansion onto the relative two-body dynamics.

To leading order, the global expansion is described by the simple linear Hubble law, $\dot{R}=H R$, which states that the relative radial velocity of two comoving objects at a mutual distance $R$ grows proportional to that distance. More precisely, the term 'distance' is here understood as the geodesic distance in the spacetime hypersurface of constant cosmological time $t$ between its two intersection points with the two worldlines of the objects considered. $H$ denotes the Hubble parameter, which generally depends on $t$ but not on space. It is given in terms of the scale parameter, $a(t)$, via $H=\dot{a} / a$.

Taking into account $\dot{H}=(\ddot{a} / a)-H^{2}$, the acceleration that results from the Hubble law is simply given by

$$
\left.\ddot{R}\right|_{\text {cosm.acc. }}=\dot{H} R+H \dot{R}=\frac{\ddot{a}}{a} R=-q H^{2} R,
$$

where

$$
q:=-\frac{\ddot{a} a}{\dot{a}^{2}}=-\frac{\ddot{a}}{a} H^{-2}
$$

is the dimensionless deceleration parameter. To get a feeling for the magnitude, we remark that for the current best-estimates for the parameters $H$ and $q, H_{0} \approx$ $70 \mathrm{Km} \cdot \mathrm{s}^{-1} \cdot \mathrm{Mpc}^{-1}$ and $q_{0} \approx-0.6$ respectively, we get $\ddot{a} / a \approx 3 \times 10^{-36} \mathrm{~s}^{-2}$, which even at Pluto's distance of 40 AU merely amounts to a tiny outward pointing acceleration of $2 \times 10^{-23} \mathrm{~m} \cdot \mathrm{s}^{-1}$.

Now note that, in the sense of General Relativity, a body that is comoving with the cosmological expansion is moving on an inertial trajectory, i.e. it is force free. On the other hand, according to Newton, a dynamical force is, by definition, the cause for deviations from inertial motion. In the present context this would mean that dynamical forces are the causes for deviations from the motions described by (7), which suggests that in Newton's law, $m \ddot{\vec{x}}=\vec{F}$, we should make the replacement

$$
\ddot{R} \mapsto \ddot{R}-(\ddot{a} / a) R
$$

in order to apply to the (sufficiently slow) motion of interacting point masses in an expanding universe. Note that this also applies to gravitational interactions in a Newtonian approximation in which gravity is considered to be a force in the above sense.

As we will see, the replacement (9) can be justified rigorously in a variety of contexts, like for gravitationally bound systems, using the equation of geodesic deviation in General Relativity. Whenever we attempt to justify the replacement (9) we must not forget that the Newtonian equations of motion (without Coriolis and centrifugal type 'forces' in them) necessarily refer to preferred systems of coordinates which are 1) locally non rotating, 2) whose origin is freely falling, and 3) in which the coordinate values directly refer to (local) inertial time (time coordinate) and spatial geodesic distance (space coordinates), as measured by comoving clocks and rods. This is achieved by using so-called Fermi normal coordinates (see, e.g., $\$ 13.6$ of (Misner et al., 1973)) in a neighborhood of a geodesic worldline - e.g. that of the Sun or the proton. This is also the approach followed in (Cooperstock et al., 1998). Note that a Fermi system of coordinates can be defined for worldlines of arbitrary acceleration and correspond to locally non-rotating frames, which may physically be realized by a system of at least two non collinear gyros in torque-free suspensions taken along the worldline. Along a geodesic, that is a worldline of zero acceleration, this system corresponds to a local inertial observer and is called Fermi normal. The equation of geodesic deviation in these coordinates now gives the variation of the spatial geodesic distance to a neighboring geodesically moving object, e.g. a planet or spacecraft. It reads ${ }^{7}$

$$
\frac{d^{2} x^{k}}{d \tau^{2}}+R_{0 l 0}^{k} x^{l}=0 .
$$

Here the $x^{k}$ are the spatial non-rotating normal coordinates whose values directly refer to the proper spatial distance. In these coordinates we further have (Cooperstock et al., 1998)

$$
R_{0 l 0}^{k}=-\delta_{l}^{k} \ddot{a} / a
$$

on the worldline of the first observer, where the overdot refers to differentiation with respect to the cosmological

\footnotetext{
7 By construction of the coordinates, the Christoffel symbols $\Gamma_{\alpha \beta}^{\mu}$ vanish along the worldline of the first observer. Since this worldline is geodesic, Fermi-Walker transportation just reduces to parallel transportation.
} 
time, which reduces to the proper time along the observer's worldline.

Equations (1011) simply state that in Fermi normal coordinates around one inertial observer another nearby inertial observer is radially accelerating away at a magnitude $(\ddot{a} / a) R$, just as envisaged before. In linear approximation, this acceleration has to be added to that resulting from the other metric perturbation that is caused by the mass at the position of the first observer. As a result, in the case of purely gravitational interaction, we obtain the equation of motion of a test particle (whose metric perturbation we neglect) in the gravitational field of a heavier object whose metric perturbation away from the FLRW cosmological background we approximate to linear order. In the case of charged objects, we neglect the metric perturbations caused by the masses of both charges as well as their electromagnetic field, and simply take into account their mutual electromagnetic interaction.

Neglecting large velocity effects (i.e. terms quadratic or higher order in $v / c$ ) we can now write down the equation of motion for the familiar two-body problem. After specification of a scale function $a(t)$, we get two ODEs for the variables $(R, \varphi)$, which describe the position ${ }^{8}$ of the orbiting body with respect to the central one:

$$
\begin{aligned}
& \ddot{R}=\frac{L^{2}}{R^{3}}-\frac{C}{R^{2}}+\frac{\ddot{a}}{a} R \\
& R^{2} \dot{\varphi}=L .
\end{aligned}
$$

These are the $(\ddot{a} / a)$-improved Newtonian equations of motion for the two-body problem, where $L$ represents the (conserved) angular momentum of the planet (or electron) per unit mass and $C$ the strength of the attractive force. In the gravitational case $C=G M$, where $M$ is the mass of the central body, and in the electromagnetic case, for the electron-proton system, $C=e^{2} / m$ (Gaussian unit), where $e$ and $m$ are the electron's charge and mass, respectively. In Sections [V] and IV we will show how to obtain (12) in appropriate limits from the full general relativistic treatments.

We now wish to study the effect the $\ddot{a}$ term has on the unperturbed Kepler orbits. We start with the obvious remark that this term results from the acceleration and not just the expansion of the universe.

Next we point out that in the concrete physical cases of interest, the time dependence of this term is negligible to a very good approximation. Indeed, putting $f:=\ddot{a} / a$, the relative time variation of the coefficient of $R$ in (7) is $\dot{f} / f$. For an exponential scale function $a(t) \propto \exp (\lambda t)$ (vacuum-energy-dominated universe) this vanishes, and for a power law $a(t) \propto t^{\lambda}$ (for example matter-, or radiation-dominated universes) this is

\footnotetext{
8 Recall that 'position' refers to Fermi normal coordinates, i.e. $R$ is the radial geodesic distance to the observer at $R=0$.
}

$-2 H / \lambda$, and hence of the order of the inverse age of the universe. If we consider a planet in the Solar System, the relevant time scale of the problem is the period of its orbit around the Sun. The relative error in the disturbance, when treating the factor $\ddot{a} / a$ as constant during an orbit, is hence smaller than $10^{-9}$. For atoms it is much smaller, of course. In principle, a time varying $\ddot{a} / a$ causes changes in the semi-major axis and eccentricity of Kepler orbits (Sereno and Jetzer, 2007). But here we shall neglect the time-dependence of (17) and set $\ddot{a} / a$ equal to a constant $A$. Because of (8) we have $A:=-q_{0} H_{0}^{2}$. Then (12a) can be immediately integrated:

$$
\frac{1}{2} \dot{R}^{2}+U(R)=E
$$

where the effective potential is

$$
U(R)=\frac{L^{2}}{2 R^{2}}-\frac{C}{R}-\frac{A}{2} R^{2} .
$$

We will see below that the three parameters $(L, C, A)$ can be effectively reduced to two.

\section{B. Specifying the initial-value problem}

Solutions of (13) and (12b) are specified by initial conditions $(R, \dot{R}, \varphi, \dot{\varphi})\left(t_{0}\right)=\left(R_{0}, V_{0}, \varphi_{0}, \omega_{0}\right)$ at the initial time $t_{0}$. The discussion of the dynamical behavior of $R$ is most effectively done in terms of the effective potential. Moreover, since perturbations are best discussed in terms of dimensionless parameters, we also introduce a length scale and a time scale that appropriately characterize the dynamical perturbation and the solution to be perturbed.

The length scale is defined as the radius at which the acceleration due to the cosmological expansion has the same magnitude as the two-body attraction. This happens precisely at the critical radius

$$
R_{c}:=\left(\frac{C}{|A|}\right)^{1 / 3} .
$$

For $R<R_{c}$ the two-body attraction dominates, whereas for $R>R_{c}$ the effect of the cosmological expansion is the dominant one.

In order to gain an understanding of the length scales of the critical radius it is instructive to express it in terms of the physical parameters. In the case of gravitational interaction we have $C /|A|=G M /\left(\left|q_{0}\right| H_{0}^{2}\right)$ and thus

$$
R_{c}=\left(\frac{R_{S} R_{H}^{2}}{2\left|q_{0}\right|}\right)^{1 / 3} .
$$

Inserting the approximate value $q_{0}=-1 / 2$ of the present epoch, this reduces to the Schücking radius (4).

In the electromagnetic case, e.g. for an electron-proton system, we have $C /|A|=\left(e^{2} / m\right) /\left(\left|q_{0}\right| H_{0}^{2}\right)$. Defining, in 
analogy with (5), the length scale

$$
R_{e}:=\frac{2 e^{2}}{m c^{2}} \approx 5.64 \cdot 10^{-15} \mathrm{~m},
$$

the critical radius (15) becomes

$$
R_{c}=\left(\frac{R_{e} R_{H}^{2}}{2\left|q_{0}\right|}\right)^{1 / 3} \approx 30 \mathrm{AU}
$$

where in the last step we inserted $q_{0}=-1 / 2$. This is about as big as the Neptune orbit!

From (16) and (18) one sees that, in both cases, a larger (smaller) $\left|q_{0}\right|$ implies a smaller (larger) critical radius, according to expectations.

So much for the length scale. The time scale is defined to be the period of the unperturbed Kepler orbit (a solution to the above problem for $A=0$ ) of semi-major axis $R_{0}$. By Kepler's third law it is given by

$$
T_{K}:=2 \pi\left(\frac{R_{0}^{3}}{C}\right)^{1 / 2}
$$

It is convenient to introduce two dimensionless parameters which essentially encode the initial conditions $R_{0}$ and $\omega_{0}$.

$$
\begin{aligned}
& \lambda:=\left(\frac{\omega_{0}}{2 \pi / T_{K}}\right)^{2}=\frac{L^{2}}{C R_{0}}, \\
& \alpha:=\operatorname{sign}(A)\left(\frac{R_{0}}{R_{c}}\right)^{3}=A \frac{R_{0}^{3}}{C} .
\end{aligned}
$$

For close to Keplerian orbits $\lambda$ is close to one. For reasonably sized orbits $\alpha$ is close to zero. For example, in the Solar System, where $R_{0}<100 \mathrm{AU}$, one has $|\alpha|<10^{-16}$. For an atom whose radius is smaller than $10^{4}$ Bohr-radii we have $|\alpha|<10^{-57}$.

Now, defining

$$
x(t):=R(t) / R_{0},
$$

equations (13) and (12b) can be written as

$$
\begin{aligned}
& \frac{1}{2} \dot{x}^{2}+\left(2 \pi / T_{K}\right)^{2} u_{\lambda, \alpha}(x)=e \\
& x^{2} \dot{\varphi}=\omega_{0},
\end{aligned}
$$

where $e:=E / r_{0}^{2}$ now plays the role of the energyconstant and where the reduced two-parameter effective potential $u_{\lambda, \alpha}$ is given by

$$
u_{\lambda, \alpha}(x):=\frac{\lambda}{2 x^{2}}-\frac{1}{x}-\frac{\alpha}{2} x^{2} .
$$

The initial conditions now read

$$
(x, \dot{x}, \varphi, \dot{\varphi})\left(t_{0}\right)=\left(1, V_{0} / R_{0}, \varphi_{0}, \omega_{0}\right) .
$$

The point of introducing the dimensionless variables is that the three initial parameters $(L, C, A)$ of the effective potential could be reduced to two: $\lambda$ and $\alpha$. This will be convenient in the discussion of the potential.

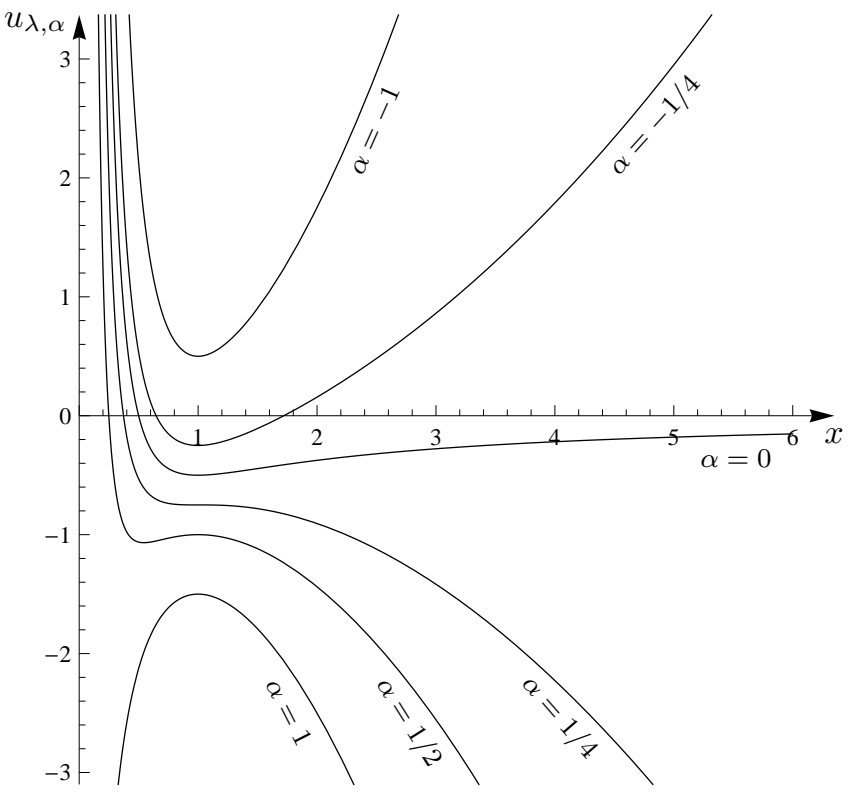

FIG. 1 The figure shows the effective potential $u_{\lambda, \alpha}$ for circular orbits (for which $\lambda=1-\alpha$ ) for some values of $\alpha$. The initial conditions are $x=1$ and $\dot{x}=0$ (see (22)). At $x=1$ the potential has an extremum, which for $\alpha<1 / 4$ is a local minimum corresponding to stable circular orbits. For $1 / 4 \leq \alpha<1$ these become unstable. The value $\alpha=0$ corresponds to the Newtonian case.

\section{Discussion of the reduced effective potential}

Circular orbits correspond to extrema of the effective potential (14). Expressed in terms of the dimensionless variables this is equivalent to $u_{\lambda, \alpha}^{\prime}(1)=-\lambda+1-\alpha=0$. By its very definition (20), $\lambda$ is always non-negative, implying $\alpha \leq 1$. For negative $\alpha$ (decelerating case) this is always satisfied. On the contrary, for positive $\alpha$ (accelerating case), this implies, in view of (21), the existence of a critical radius, given by $R_{c}$, beyond which no circular orbit exists. These orbits are stable if the considered extremum is a true minimum, i.e. if the second derivative of the potential evaluated at the critical value is positive. Now, $u_{\lambda, \alpha}^{\prime \prime}(1)=3 \lambda-2-\alpha=1-4 \alpha$, showing stability for $\alpha<1 / 4$ and instability for $\alpha \geq 1 / 4$. For the accelerating case, in view of (21), this implies that the circular orbits are stable iff $R_{0}$ is smaller than the critical value

$$
R_{\mathrm{sco}}:=(1 / 4)^{1 / 3} R_{c} \approx 0.63 R_{c},
$$

where 'sco' stands for 'stable circular orbits'.

Summarizing, we have the following situation: in the decelerating case (i.e. for negative $\alpha$ or, equivalently, for negative $A$ ) stable circular orbits exist for every radius $R_{0}$; one just has to increase the angular velocity by some amount stated below in (28). On the contrary, in the accelerating case (i.e. for positive $\alpha$, or, equivalently, for positive $A$ ), we have three regions:

- $R_{0}<R_{\text {sco }}$, where circular orbits exist and are stable, 
- $R_{\mathrm{sco}} \leq R_{0} \leq R_{c}$, where circular orbits exist but are unstable, and

- $R_{0}>R_{c}$, where no circular orbits exist.

Generally, there exist no bounded orbits that extend beyond the critical radius $R_{c}$, the reason being simply that there is no $R>R_{c}$ where $U^{\prime}(R)>0$. Bigger systems will just be slowly pulled apart by the cosmological acceleration and approximately move with the Hubble flow at later times. ${ }^{9}$ Modifications of this strict qualitative distinction implied by time dependencies of $A$ in (14) were discussed in (Faraoni and Jacques, 2007).

Turning back to the case of circular orbits, we now express the condition for an extrema derived above, $\lambda=$ $1-\alpha$, in terms of the physical quantities, which leads to

$$
\omega_{0}=\left(2 \pi / T_{K}\right) \sqrt{1-\operatorname{sign}(A)\left(R_{0} / R_{c}\right)^{3}} .
$$

This equation says that, in order to get a circular orbit, our planet, or electron, must have a smaller or bigger angular velocity according to the universe expanding in an accelerating or decelerating fashion, respectively. This is just what one would expect, since the effect of a cosmological 'pulling apart' or 'pushing together' must be compensated by a smaller or larger centrifugal forces respectively, as compared to the Keplerian case. Equation (28) represents a modification of the third Kepler law due to the cosmological expansion. In principle this is measurable, but it is an effect of order $\left(R_{0} / R_{c}\right)^{3}$ and hence very small indeed; e.g. smaller than $10^{-17}$ for a planet in the Solar System.

Instead of adjusting the initial angular velocity as in (28), we can ask how one has to modify $r_{0}$ in order to get a circular orbit with the angular velocity $\omega_{0}=2 \pi / T_{K}$. This is equivalent to searching the minimum of the effective potential (25) for $\lambda=1$. This condition leads to the fourth order equation $\alpha x^{4}-x+1=0$ with respect to $x$. Its solutions can be exactly written down using Ferrari's formula, though this is not illuminating. For our purposes it is more convenient to solve it approximately, treating $\alpha$ as a small perturbation. Inserting the ansatz $x_{\min }=c_{0}+c_{1} \alpha+\mathcal{O}\left(\alpha^{2}\right)$ we get $c_{0}=c_{1}=1$. This is really a minimum since $u_{1, \alpha}^{\prime \prime}\left(x_{\min }\right)=1+\mathcal{O}(\alpha)>0$. Hence we have

$$
R_{\min }=R_{0}\left(1+\operatorname{sign}(A)\left(\frac{R_{0}}{R_{c}}\right)^{3}+\mathcal{O}\left(\left(R_{0} / R_{c}\right)^{6}\right)\right)
$$

This tells us that in the accelerating (decelerating) case the radii of the circular orbits with $\omega_{0}=2 \pi / T_{K}$ becomes bigger (smaller), again according to physical expectation. As an example, the deviation in the radius for an hypothetical spacecraft orbiting around the Sun

\footnotetext{
9 This genuine non-perturbative behavior was not seen in the perturbation analysis performed in (Cooperstock et al., 1998).
}

at $100 \mathrm{AU}$ would be just of the order of $1 \mathrm{~mm}$. Since it grows with the fourth power of the distance, the deviation at $1000 \mathrm{AU}$ would be of the order of 10 meters.

\section{GENERAL-RELATIVISTIC TREATMENT FOR ELECTROMAGNETICALLY-BOUNDED SYSTEMS}

In this section we show how to arrive at (12) from a relativistic treatment of an electromagnetically bounded two-body system embedded (without back-reaction) into an expanding (spatially flat) universe. This implies solving Maxwell's equations in the cosmological background (30) for an electric point charge (the proton) and then integrate the Lorentz equations for the motion of a particle (electron) in a bound orbit (cf. (Bonnor, 1999)). Equation (12) then appears in an appropriate slow-motion limit. However, in order to relate this straightforward method to a famous argument of Dicke \& Peebles, we shall proceed by taking a slight detour which makes use of the conformal properties of Maxwell's equations.

\section{A. The argument of Dicke and Peebles}

In reference (Dicke and Peebles, 1964) Dicke \& Peebles presented an apparently very general and elegant argument that purports to show the insignificance of any dynamical effect of cosmological expansion on a local system that is either bound by electromagnetic or gravitational forces and which should hold true at any scale. Their argument involves a rescaling of spacetime coordinates, $(t, \vec{x}) \mapsto(\lambda t, \lambda \vec{x})$ and certain assumptions on how other physical quantities, most prominently mass, behave under such scaling transformations. For example, they assume mass to transform like $m \mapsto \lambda^{-1} m$. However, their argument is really independent of such assumptions, as we shall show below. We work from first principles to clearly display all assumptions made.

We consider the motion of a charged point particle in an electromagnetic field. The whole system, i.e. particle plus electromagnetic field, is placed into a cosmological FLRW-spacetime with flat $(k=0)$ spatial geometry. The spacetime metric reads

$$
\boldsymbol{g}=c^{2} \boldsymbol{d} t^{2}-a^{2}(t)\left(\boldsymbol{d} r^{2}+r^{2} \boldsymbol{g}_{S^{2}}\right),
$$

where

$$
\boldsymbol{g}_{S^{2}}=\boldsymbol{d} \theta^{2}+\sin ^{2} \theta \boldsymbol{d} \varphi^{2}
$$

denotes the metric on the unit two-sphere in standard coordinates. We introduce conformal time, $t_{c}$, via

$$
t_{c}=f(t):=\int_{k}^{t} \frac{d t^{\prime}}{a\left(t^{\prime}\right)},
$$

by means of which we can write (30) in a conformally flat form

$$
\boldsymbol{g}=a_{c}^{2}\left(t_{c}\right)\left(c^{2} \boldsymbol{d} t_{c}^{2}-\boldsymbol{d} r^{2}-r^{2} \boldsymbol{g}_{S^{2}}\right)=a_{c}^{2}\left(t_{c}\right) \boldsymbol{\eta},
$$


where $\boldsymbol{\eta}$ denotes the flat Minkowski metric. Here we wrote $a_{c}$ to indicate that we now expressed the expansion parameter $a$ as function of $t_{c}$ rather than $t$, i.e.

$$
a_{c}:=a \circ f^{-1} .
$$

The electromagnetic field is characterized by the tensor $F_{\mu \nu}$, comprising electric and magnetic fields:

$$
F_{\mu \nu}=\left(\begin{array}{cc}
0 & E_{n} / c \\
-E_{m} / c & -\varepsilon_{m n j} B_{j}
\end{array}\right) .
$$

In terms of the electromagnetic four-vector potential, $A_{\mu}=(\varphi / c,-\vec{A})$, one has

$$
F_{\mu \nu}=\partial_{\mu} A_{\nu}-\partial_{\nu} A_{\mu}=\nabla_{\mu} A_{\nu}-\nabla_{\nu} A_{\mu}
$$

so that, as usual, $\vec{E}=-\vec{\nabla} \phi-\dot{\vec{A}}$. The expression for the four-vector of the Lorentz-force of a particle of charge $e$ moving in the field $F_{\mu \nu}$ is $e F^{\mu}{ }_{\nu} u^{\nu}$, where $u^{\mu}$ is the particle's four velocity.

The equations of motion for the system Particle + EMField follow from an action which is the sum of the action of the particle, the action for its interaction with the electromagnetic field, and the action for the free field, all placed in the background (30). Hence we write:

$$
S=S_{P}+S_{I}+S_{F},
$$

where

$$
\begin{aligned}
S_{P} & =-m c^{2} \int_{z} d \tau=-m c \int \sqrt{g\left(z^{\prime}, z^{\prime}\right)} d \lambda \\
S_{I} & =-e \int A_{z} d x^{\mu}=-e \int A_{\mu}(z(\lambda)) z^{\prime \mu} d \lambda \\
& =-\int d^{4} x A_{\mu}(x) \int d \lambda e \delta^{(4)}(x-z(\lambda)) z^{\prime \mu}, \\
S_{F} & =\frac{-1}{4} \int d^{4} x \sqrt{-g} g^{\mu \alpha} g^{\nu \beta} F_{\mu \nu} F_{\alpha \beta} \\
& =\frac{-1}{4} \int d^{4} x \eta^{\mu \alpha} \eta^{\nu \beta} F_{\mu \nu} F_{\alpha \beta} .
\end{aligned}
$$

Here $\lambda$ is an arbitrary parameter along the worldline $z$ : $\lambda \mapsto z(\lambda)$ of the particle, and $z^{\prime}$ the derivative $d z / d \lambda$. The differential of the proper time along this worldline is

$$
d \tau=\sqrt{g\left(z^{\prime}, z^{\prime}\right)} d \lambda=\sqrt{g_{\mu \nu}(z(\lambda)) \frac{d z^{\mu}}{d \lambda} \frac{d z^{\nu}}{d \lambda}} d \lambda .
$$

It is now important to note that 1$)$ the background metric $g$ does not enter (38b) and that (38c) is conformally invariant (in 4 spacetime dimensions only!). Hence the expansion factor, $a\left(t_{c}\right)$, does not enter these two expressions. For this reason we could write (38c) in terms of the flat Minkowski metric, though it should be kept in mind that the time coordinate is now given by conformal time $t_{c}$. This is not the time read by standard clocks that move with the cosmological observers, which rather show the cosmological time $t$ (which is the proper time along the geodesic flow of the observer field $\boldsymbol{\partial} / \boldsymbol{\partial} t)$.

The situation is rather different for the action (38a) of the particle. Its variational derivative with respect to $z(\lambda)$ is

$$
\frac{\delta S_{p}}{\delta z^{\mu}(\lambda)}=-m c\left\{\frac{\frac{1}{2} g_{\alpha \beta, \mu} z^{\prime \alpha} z^{\prime \beta}}{\sqrt{g\left(z^{\prime}, z^{\prime}\right)}}-\frac{d}{d \lambda}\left[\frac{g_{\mu \alpha} z^{\prime \alpha}}{\sqrt{g\left(z^{\prime}, z^{\prime}\right)}}\right]\right\}
$$

We now introduce the conformal proper time, $\tau_{c}$, via

$$
d \tau_{c}=(1 / c) \sqrt{\eta\left(z^{\prime}, z^{\prime}\right)} d \lambda=(1 / c a) \sqrt{g\left(z^{\prime}, z^{\prime}\right)} d \lambda .
$$

We denote differentiation with respect to $\tau_{c}$ by an overdot, so that e.g. $z^{\prime} / \sqrt{g\left(z^{\prime}, z^{\prime}\right)}=\dot{z} / c a$. Using this to replace $z^{\prime}$ by $\dot{z} \sqrt{g\left(z^{\prime}, z^{\prime}\right)} / c a$ and also $g$ by $a^{2} \eta$ in (40) gives

$$
\frac{\delta S_{p}}{\delta z^{\mu}(\lambda)}=\frac{\sqrt{g\left(z^{\prime}, z^{\prime}\right)}}{a c} m a\left\{\eta_{\mu \alpha} \ddot{z}^{\alpha}-P_{\mu}^{\alpha} \phi, \alpha\right\}
$$

where we set

$$
a=: \exp \left(\phi / c^{2}\right) \quad \text { and } \quad P_{\mu}^{\alpha}:=\delta_{\mu}^{\alpha}-\frac{\dot{z}^{\alpha} \dot{z}^{\nu}}{c^{2}} \eta_{\nu \mu} .
$$

Recalling that $\delta S_{P}=\int \frac{\delta S_{p}}{\delta z^{\mu}(\lambda)} \delta z^{\mu} d \lambda=\int \frac{\delta S_{p}}{\delta z^{\mu}\left(\tau_{c}\right)} \delta z^{\mu} d \tau_{c}$ and using (41), (42) is equivalent to

$$
\frac{\delta S_{p}}{\delta z^{\mu}\left(\tau_{c}\right)}=m a\left(\ddot{z}^{\alpha}-P_{\mu}^{\alpha} \phi, \alpha\right)
$$

where from now on we agree to raise and lower indices using the Minkowski metric, i.e. $\eta_{\mu \nu}=\operatorname{diag}(1,-1,-1,-1)$ in Minkowski inertial coordinates.

Writing (38b) in terms of the conformal proper time and taking the variational derivative with respect to $z\left(\tau_{c}\right)$ leads to $\delta S_{I} / \delta z^{\mu}\left(\tau_{c}\right)=-e F_{\mu \alpha} \dot{z}^{\alpha}$, so that

$$
\frac{\delta S}{\delta z^{\mu}\left(\tau_{c}\right)}=m a\left(\ddot{z}_{\mu}-P_{\mu}^{\alpha} \phi_{, \alpha}\right)-e F_{\mu \alpha} \dot{z}^{\alpha} .
$$

The variational derivative of the action with respect to the vector potential $A$ is

$$
\frac{\delta S}{\delta A_{\mu}(x)}=\partial_{\alpha} F^{\mu \alpha}(x)-e \int d \tau_{c} \delta\left(x-z\left(\tau_{c}\right)\right) \dot{z}^{\mu}\left(\tau_{c}\right) .
$$

Equations (45) and (46) show that the fully dynamical problem can be treated as if it were situated in static flat space. The field equations that follow from (46) are just the same as in Minkowski space. Hence we can calculate the Coulomb field as usual. On the other hand, the equations of motion receive two changes from the cosmological expansion term: the first is that the mass $m$ is now multiplied with the (time-dependent!) scale factor $a$, the second is an additional scalar force induced by a. Note that all spacetime dependent functions on the right hand side are to be evaluated at the particle's location $z\left(\tau_{c}\right)$, whose fourth component corresponds to $c t_{c}$. 
Hence, writing out all arguments and taking into account that the time coordinate is $t_{c}$, we have for the equation of motion

$$
\begin{aligned}
\ddot{z}^{\mu}= & \frac{e}{m a_{c}\left(z^{0} / c\right)} F_{\alpha}^{\mu}(z) \dot{z}^{\alpha} \\
& -\left(-c^{2} \eta^{\mu \alpha}+\dot{z}^{\mu} \dot{z}^{\alpha}\right) \partial_{\alpha} \ln a_{c}\left(z^{0} / c\right) \\
= & \frac{e}{m a_{c}\left(z^{0} / c\right)} F^{\mu}{ }_{\alpha}(z) \dot{z}^{\alpha} \\
& -\left(-c \eta^{\mu 0}+\dot{z}^{\mu} \dot{z}^{0} / c\right) a_{c}^{\prime}\left(z^{0} / c\right) / a_{c}\left(z^{0} / c\right),
\end{aligned}
$$

where $a_{c}^{\prime}$ is the derivative of $a_{c}$.

So far no approximations were made. Now we write $\dot{z}^{\mu}=\gamma(c, \vec{v})$, where $\vec{v}$ is the derivative of $\vec{z}$ with respect to the conformal time $t_{c}$, henceforth denoted by a prime, and $\gamma=1 / \sqrt{1-v^{2} / c^{2}}$. Then we specialize to slow motions, i.e. neglect effects of quadratic or higher powers in $v / c$ (special relativistic effects). For the spatial part of (47b) we get

$$
\vec{z}^{\prime \prime}+\vec{z}^{\prime}\left(a_{c}^{\prime} / a_{c}\right)=\frac{e}{m a_{c}}\left(\vec{E}+\vec{z}^{\prime} \times \vec{B}\right),
$$

where we once more recall that the spatial coordinates used here are the comoving (i.e. conformal) ones and the electric and magnetic fields are evaluated at the particle's position $\vec{z}\left(t_{c}\right)$.

From the above equation we see that the effect of cosmological expansion in the conformal coordinates shows up in two ways: first in a time dependence of the mass which scales with $a_{c}$, and, second, in the presence a friction term. Dicke \& Peebles neglect the friction term and simply conclude as follows: In the adiabatic approximation, which is justified if typical time scales of the problem at hand are short compared to the world-age (corresponding to small $\varepsilon_{2}$ in (98b)), the time-dependent mass term leads to a time varying radius in comoving (or conformal) coordinates of $r\left(t_{c}\right) \propto 1 / a_{c}\left(t_{c}\right)$. Hence the physical radius (given by the cosmological geodesically spatial distance), $r_{*}=a_{c} r$, stays constant in this approximation. Hence, within this approximation, electromagnetically bound systems do not feel any effect of cosmological expansion.

But what does 'this approximation' refer to? We will see that it amounts to neglecting precisely the leading order contributions. This is easy to see if we cast (48) into physical coordinates, given by the cosmological time $t$ and the cosmological geodesic spatial distance $r_{*}:=$ $a(t) r$. We have $d t_{c} / d t=1 / a$ and the spatial geodesic coordinates are $\vec{y}:=a(t) \vec{z}$. Denoting by an overdot the time derivative with respect to $t$, the two terms on the left hand side of (48) become

$$
\begin{aligned}
\vec{z}^{\prime \prime} & =a\left(\vec{y} H^{2}-\dot{\vec{y}} H\right)+a(\ddot{\vec{y}}-\vec{y} \ddot{a} / a), \\
\vec{z}^{\prime}\left(a_{c}^{\prime} / a_{c}\right) & =-a\left(\vec{y} H^{2}-\dot{\vec{y}} H\right),
\end{aligned}
$$

where $H=\dot{a} / a$. This shows that the friction term cancels against the first-order derivative terms in $\vec{y}$ and $a$ that one gets in rewriting the left-hand side of (48) in physical coordinates. ${ }^{10}$ The only additional term next to $\ddot{\vec{y}}$ that survives is precisely the familiar acceleration term (7). Inserting (49) into (48), whose right-hand side we now specialize to a pure electric Coulomb field, $\vec{E}(\vec{z})=Q \vec{z} /|\vec{z}|^{3}$ and $\vec{B}(\vec{z})=0$, we arrive at

$$
\ddot{\vec{y}}-\vec{y}(\ddot{a} / a)=\frac{e Q}{m|\vec{y}|^{3}} \vec{y} .
$$

After introducing polar coordinates in the orbital plane we exactly get (12).

\section{B. Exact condition for non-expanding circular orbits}

In (Bonnor, 1999) a necessary and sufficient condition for the existence of non-expanding orbits is derived for the electron-proton system in a spatially flat FLRW spacetime. Here 'non-expanding' is defined as of constant areal radius. This condition follows directly from the Lorentz equation of motion for the electron in the external electric field of the proton, the normalization condition of the electron's four-velocity, and the condition of constancy of the areal radius. In our notation, introducing the dimensionless quantities $h(t):=R H(t) / c$, $l:=L / R c$, and $\mu:=R_{e} / 2 R$, the conditions for the existence of non-expanding circular orbits reads as follows:

$$
\frac{R}{c} \dot{h}=\frac{\left(1-h^{2}\right)^{3 / 2}}{\left(1+l^{2}\right)^{1 / 2}}\left(\mu-\frac{l^{2}+h^{2}}{\sqrt{\left(1+l^{2}\right)\left(1-h^{2}\right)}}\right) \text {. }
$$

Recall that $R_{e}$ is defined in (17) and $H(t)$ and $L$ denote the Hubble function and, respectively, the (conserved) electron's angular momentum per unit mass. The above condition is a first-order autonomous ODE for the function $h(t)$, and hence for the Hubble function $H(t)$. This is the constraint on the spacetime (more precisely, on the scale factor $a(t))$ that one gets by imposing the existence of non-expanding circular orbits for two oppositely charged point masses. If such orbits exist, (51) amounts to the generalization of Kepler's third law to FLRW spacetimes, which here gives a relation between the scale function on one hand and the orbital parameters $R$ and $L$ as well as the field-strength parameter $R_{e}$ on the other. Recall that in Newtonian physics the third Kepler law is, in our notation, simply given by $l^{2}=\mu$.

The easiest solutions of (51) are of course the stationary ones, that is with $h(t) \equiv h_{0}$, for some constant $h_{0}$. This means that the scale factors is exponentially expanding,

$$
a(t)=a_{0} \exp \left(H_{0} t\right)
$$

10 Since the friction term cancels, the critical remark [27] in (Adkins et al., 2007) regarding its magnitude is based on a misunderstanding. 
where $H_{0}:=h_{0} c / R$ and $a_{0}$ is some positive constant. In other words, the spacetime is given by the de Sitter solution $(\Lambda$-dominated universe). In this case (51) reduces to

$$
\frac{l^{2}+h_{0}^{2}}{\sqrt{\left(1+l^{2}\right)\left(1-h_{0}^{2}\right)}}=\mu .
$$

Notice that a larger Hubble parameter, hence a larger $h_{0}$, makes the l.h.s. larger. Consequently, (53) tells us that with a larger Hubble parameter we must give to the electron a smaller angular velocity (smaller $l$ ) in order to keep it on a non-expanding circular orbit with the same radius. This, according to intuition, is in order to compensate the extra cosmological pull with a reduced centrifugal term. In case of Minkowski spacetime $\left(h_{0}=0\right)$ the above relation reads $l^{2} / \sqrt{1+l^{2}}=\mu$, hence one can interpret the factor $1 / \sqrt{1+l^{2}}$ as a special-relativistic correction to the Newtonian relation $l^{2}=\mu$. The largest radius at which, in an FLRW spacetime with exponentiallygrowing scale factor, there is a non-expanding orbit follows from (53) in the limit $l \rightarrow 0$. In this limit the condition reduces to $h_{0}^{2} / \sqrt{1-h_{0}^{2}}=\mu$, which, for small parameters $h_{0}$, simplifies to $h_{0}^{2} \approx \mu$. Solving for $R$ this gives the radius $\left(R_{e} R_{H}^{2} / 2\right)^{1 / 3}$, which, taking into account that $q_{0}=-1$ because of (52), exactly corresponds to the critical radius (18).

The other (non-stationary) solutions of (51) can also be found. After separation of variables and an elementary integration one gets $t$ as function of $h$ in terms of trigonometric functions composed with inverse hyperbolic functions. This exact expression is again not very illuminating and cannot generally be explicitly inverted so as to obtain $h$ in terms of elementary functions of $t$. However, if we make use of the smallness of the parameters $\mu, l^{2}$, and $h^{2}$, a leading-order expansion in these quantities gives a much simpler expression for $t(h)$ which can be explicitly inverted. In fact, this approximate solution $h(t)$ is obtained much quicker by solving (51) with the right-hand side being replaced with its leading-order expansion in the mentioned quantities, that is, by solving

$$
\frac{R}{c} \dot{h}=\mu-l^{2}-h^{2} .
$$

Here $\mu-l^{2}$ is a constant which depends on the orbit parameters. One must now distinguish between three cases: (a) $\mu-l^{2}=: \kappa^{2}>0$ for some positive $\kappa$, (b) $\mu-l^{2}=$ : $-\nu^{2}<0$ for some positive $\nu$, and (c) $\mu-l^{2}=0$. Recalling the Newtonian relation $l^{2}=\mu$, orbits in the three cases have an angular momentum which is, respectively, smaller, bigger, and equal to the Newtonian one. Integrating (54) we get, putting w.l.o.g. $t_{0}=0, h(t)=$ $\kappa \tanh (\kappa c t / R), h(t)=-\nu \tan (\nu c t / R)$, and $h(t)=R / c t$, for the cases $(a),(b)$, and $(c)$, respectively. Then, integrating once and exponentiating the result, we get the corresponding scale functions:

(a) Case $\mu-l^{2}=: \kappa^{2}>0$ (non-expanding orbits have
sub-Newtonian angular momentum)

$$
a(t)=a_{0} \cosh \left(\frac{\kappa c t}{R}\right), \quad t \in[0, \infty) .
$$

(b) Case $\mu-l^{2}=:-\nu^{2}<0$ (non-expanding orbits have super-Newtonian angular momentum)

$$
a(t)=a_{0} \cos \left(\frac{\nu c t}{R}\right), \quad t \in\left[0, \frac{\pi R}{2 \nu c}\right) .
$$

(c) Case $\mu-l^{2}=0$ (non-expanding orbits have Newtonian angular momentum)

$$
a(t)=a_{0} t, \quad t \in(0, \infty) .
$$

In all three cases (a), (b), and (c) $a_{0}$ is a positive constant and the acceleration term $\ddot{a} / a$ is a constant which is positive, negative, and zero, respectively. Hence, as one would intuitively expect, the non-expanding orbits have an angular momentum which is smaller, larger, or equal the Newtonian one, depending on whether the acceleration factor $\ddot{a} / a$ is positive, negative, or zero.

\section{GENERAL-RELATIVISTIC TREATMENT FOR GRAVITATIONALLY-BOUNDED SYSTEMS}

As advertised in Section II.B, we now wish to discuss exact solutions that may represent quasi-isolated spherically-symmetric gravitating systems 'embedded' into cosmological spacetimes. As regards the meaning of 'embedded' we distinguish between the strategies of 'matching' and 'melting', as outlined in Sect.II.B.

\section{A. Spherically-symmetric matchings}

The complexity and non-linearity of Einstein's equations make it a very difficult task to construct a suitable variety of exact solutions which serve as realistic models for actual physical situations. Often exact solutions are only known for highly idealized situations, typically with high degrees of symmetry, in which the field equations sufficiently simplify. One way to construct new solutions (in a suitable sense, see below) from old ones is to glue them across suitably chosen hypersurfaces along which the matter distribution may become singular due to surface layers. This approach was pioneered by Lanczos in the early 1920s (Lanczos, 1924) and put into geometric form by Darmois (Darmois, 1927) and (Israel, 1966); see also $\S 21.13$ of (Misner et al., 1973). In this section, under the assumption of spherically symmetry, we present a new alternative set of conditions which are equivalent to the old ones. The new conditions only involve scalar quantities, are easy to verify, and have good physical interpretations. More details are contained in Carrera, 2009). 
Here we shall restrict to piecewise continuous matter distributions without singular ( $\delta$-distribution like) surface layers, as e.g. in the presence of stars with sharply defined surfaces. Einstein's equations can the be satisfied for piecewise twice continuously differentiable fields, if the field equations at the location of the matching hypersurface are replaced by their one-dimensional $\varepsilon$-interval integrals in normal direction to the hypersurface. The condition that two twice continuously differentiable solutions (in the ordinary sense) can be matched into a piecewise twice continuously differentiable solution (in the re-interpreted sense just explained) is then simply given by the so-called

Darmois junction conditions (DJC). For a nonnull matching hypersurface $\Gamma$, $(i)$ the induced metric $\boldsymbol{g}_{\Gamma}$ and (ii) the extrinsic curvature $\boldsymbol{K}_{\Gamma}$ shall be continuous through $\Gamma$.

Let us pause for a moment to say a few more words about the notion of 'continuity through $\Gamma$ '. Gluing together two pieces of spacetimes means the following: Initially one has two spacetimes, say $\left(\mathcal{M}^{+}, \boldsymbol{g}^{+}\right)$and $\left(\mathcal{M}^{-}, \boldsymbol{g}^{-}\right)$, with oriented boundaries $\Gamma^{+}$and $\Gamma^{-}$, respectively. Given a diffeomorphism $\phi: \phi: \Gamma^{+} \rightarrow \Gamma^{-}$between the boundaries, the glued spacetime is the quotient of the disjoint union of $\mathcal{M}^{+}$and $\mathcal{M}^{-}$under the identification of each point of $p \in \Gamma^{+}$with $\phi(p) \in \Gamma^{-}$. The matching hypersurface $\Gamma$ is now the common image of $\Gamma^{+}$and $\Gamma^{-}$after identification in the quotient spacetime. Now, a tensor field $\boldsymbol{T}$ is said to be continuous through $\Gamma$ if $\left.\boldsymbol{T}\right|_{\Gamma^{+}}$equals $\left.\boldsymbol{T}\right|_{\Gamma^{-}}$under the push-forward action of the diffeomorphism $\phi$, hence if $\phi_{*}\left(\left.\boldsymbol{T}\right|_{\Gamma^{+}}\right)=\left.\boldsymbol{T}\right|_{\Gamma^{-}}$.

Let us now return to the DJC and, in particular, their physical interpretation. If $\boldsymbol{n}$ is a continuous choice of unit normal of $\Gamma$, it implies that

$$
\boldsymbol{T}(\boldsymbol{n}, \cdot) \text { is continuous through } \Gamma \text {. }
$$

This follows directly from the expressions (C8) of the Einstein tensor given in Appendix C] If $\Gamma$ is timelike and hence $\boldsymbol{n}$ spacelike, (56) just states the continuity of the normal components for the energy-momentum flux-densities, whereas their tangential components together with the energy density may jump across $\Gamma$. In the absence of surface layers this continuity condition is just a physically obvious consequence of local energymomentum conservation, whereas jumps in, say, the energy-density must clearly be allowed for. For completeness we note that for a spacelike matching surface (56) states the continuity of the densities of energy and momentum as measured by an observer moving along $\boldsymbol{n}$ (taken to be future pointing), whereas the corresponding currents may jump.

Let now restrict our attention to spherical symmetric spacetimes glued along hypersurfaces of spherical symmetry. This means that the latter are left invariant, as set, under the action of $S O(3)$. We recall that the structure of a spherically symmetric spacetime is that of a warped product $\mathcal{M}=\mathcal{B} \times_{R} S^{2}$ of a two-dimensional Lorentzian manifold $\mathcal{B}$ and the two-sphere by means of the warping function, $R: \mathcal{B} \rightarrow \mathbb{R}_{+}$, called the areal radius. The matching hypersurfaces are then of the form $\Gamma=\gamma \times{ }_{R} S^{2}$, where $\gamma$ is the projection of $\Gamma$ into $\mathcal{B}$ and is called the matching curve. The DJC should then reduce to appropriate conditions along the curve $\gamma$. Indeed, Theorem 1 below shows that, in the spherically symmetric case, the DJC are equivalent to the following

Spherically symmetric junction conditions (SSJC). Let $\Gamma$ be a smooth, non-null, spherically symmetric matching hypersurface between two spherically symmetric spacetimes and $\boldsymbol{n}$ a continuous choice of unit normal vector field on $\Gamma$. Denote with $\gamma$ the projection of $\Gamma$ onto $\mathcal{B}$. Moreover, let $\boldsymbol{v}$ the (unique up to a sign) spherically symmetric, unit vector field on $\Gamma$ orthogonal to $\boldsymbol{n}$. The following four functions

(i) the arc-length of $\gamma$,

(ii) the extrinsic curvature of $\gamma$ in $\mathcal{B}: \boldsymbol{g}\left(\boldsymbol{n}, \nabla_{\boldsymbol{v}} \boldsymbol{v}\right)$,

(iii) the areal radius $R$,

(iv) the Misner-Sharp energy $E$, shall be continuous through the matching curve $\gamma$.

The Misner-Sharp (MS) energy (Misner and Sharp, 1964) and (Hernandez and Misner, 1966), which we will denote by $E$, is a concept of quasi-local mass that can be defined in presence of spherical symmetry, and which proves useful for computational and interpretational purposes. It is a function defined in purely geometrical manner as follows. Given a point $p$ of spacetime, compute the sectional curvature $K$ of the plane tangent to the two-dimensional $S O(3)$-orbit through $p$ and multiply this with minus ${ }^{11}$ one-half of the third power of the areal radius:

$$
E:=-\frac{1}{2} R^{3} K
$$

From (D9) we immediately read off

$$
E=\frac{R}{2}(1+\langle\boldsymbol{d} R, \boldsymbol{d} R\rangle)
$$

which provides a convenient expression for the computation of the MS energy. We shall show in Appendix[D that the MS energy is the charge of a conserved current and how it depends on the energy-momentum tensor for the matter. There we will also briefly discuss its Newtonian limit. This allows to interpret it as amount of active gravitational energy contained in the interior of the sphere of symmetry $(S O(3)$-orbit) through $p$. There we also show that the MS energy at $p$ is equal to the Hawking quasilocal mass of the two-sphere of symmetry ( $S O(3)$-orbit) through $p$ and hence converges to the Bondi-mass at null infinity and, in an asymptotically flat spacetime, to the ADM mass at spatial infinity (for the latter two issues

11 The minus sign here is just a relict of our signature choice. 
see (Szabados, 2004) and also (Hayward, 1996)). Moreover, we will give the decomposition of the MS energy in its Ricci and Weyl parts; see (D34).

The name 'Misner-Sharp energy' seems now to be established in the literature, however one should say that this mass concept goes back at least to (Lemaitre, $1933)^{12}$, which gives a coordinate expression for it. Its geometric definition (57) was first given in (Hernandez and Misner, 1966) and its interpretation as the charge of a conserved current was first derived in (Kodama, 1980). Later, an alternative definition was given in (Zannias, 1990): There it is showed that the MS energy can defined in terms of the norm of the Killing fields generating the isometry group $S O(3)$, leading directly to (58). Further relevant studies of the MS energy are (Cahill and McVittie, 1970a.b) and, more recently, (Burnett, 1991; Hayward, 1996, 1998).

Some comment are needed on the above SSJC. First we note that, since $\boldsymbol{n}$ and $\boldsymbol{v}$ are spherically symmetric and hence tangent to $\mathcal{B}$ we have in view of (D3) and (C6) that $\boldsymbol{g}\left(\boldsymbol{n}, \boldsymbol{\nabla}_{\boldsymbol{v}} \boldsymbol{v}\right)=\boldsymbol{g}_{\mathcal{B}}\left(\boldsymbol{n}_{\mathcal{B}},{ }^{\mathcal{B}} \boldsymbol{\nabla}_{\boldsymbol{v}_{\mathcal{B}}} \boldsymbol{v}_{\mathcal{B}}\right)=\varepsilon\left(\boldsymbol{n}_{\mathcal{B}}\right) \boldsymbol{K}_{\gamma}^{\mathcal{B}}\left(\boldsymbol{v}_{\mathcal{B}}, \boldsymbol{v}_{\mathcal{B}}\right)$. Hence, the quantity in (ii) is indeed (up to a possible sign) the extrinsic curvature of the curve $\gamma$ in $\mathcal{B}$. Second, note that this quantity, being quadratic in $\boldsymbol{v}$, does not depend on the sign choice of $\boldsymbol{v}$. Third, since the matching hypersurface has the structure $\gamma \times S^{2}$, the words 'continuous through the curve $\gamma^{\prime}$ can be interchanged with the words 'continuous through the hypersurface $\Gamma$ ', depending on ones preference to think four- or two-dimensional.

A great advantage of the SSJC is that they are very easy to verify: one simply has to impose continuity on four scalars along the matching curve in the twodimensional base manifold $\mathcal{B}$. Dealing with scalars, since their value is independent on the particular coordinate choice, one does not need to worry about introducing new coordinates in both spacetimes to be glued, in order to get the different metrics in a form which is comparable. This is indeed an ingrate task: in general, these coordinates are only needed in order to check if the junction conditions are satisfied, and for nothing more. In presence of spherical symmetry all this can be circumvented by using our new junction conditions.

Furthermore, the SSJC have a good physical interpretation: The continuity condition of both, the areal radius as well as of the MS energy, can be read as equilibrium condition for the gravitational pull acting from opposite directions onto (fictitious) test masses at the location of the matching surface. Concerning the continuity of the extrinsic curvature of the matching curve, we note the following: In the case where the matching hypersurface $\Gamma$ is timelike, let $\gamma=\pi(\Gamma)$ be a timelike curve in $\mathcal{B}$ and $\boldsymbol{v}$ is future-pointing tangent. One can think of $\gamma$ as the 'matching observer's' worldline. Hence, in the timelike case, the extrinsic curvature is nothing but the acceler-

\footnotetext{
${ }^{12}$ For an English translation see (Lemaître, 1997).
}

ation of the matching observer. In the spatial case, on the contrary, $\boldsymbol{n}$ is timelike, $\gamma$ is spacelike (and $\boldsymbol{v}$ tangent to it). One can choose $\boldsymbol{n}$ to be future-pointing and then think of it as an observer field defined along the spatial (1-dimensional) slice $\gamma$. In view of (C7) one then sees that the extrinsic curvature of $\gamma$ is exactly ${ }^{13}$ the shearexpansion of $\boldsymbol{n}$ in direction of $\boldsymbol{v}$ 'radial direction'.

In Appendix $\mathrm{B}$ we prove the following

Theorem 1 (Equivalence of the junction conditions). Let $\Gamma$ be a smooth, non-null, spherically symmetric matching hypersurface between two spherically symmetric spacetimes and $\boldsymbol{n}$ a continuous choice of unit normal vector field on $\Gamma$. Assume, moreover, that the areal radii of the two spacetimes are $C^{1}$ functions in an open neighborhood of the matching hypersurfaces. Then the DJC are equivalent to the SSJC.

Now let us suppose we are faced with the following situation: We are given two spherically symmetric solutions of Einstein equation and we want to know if they can be matched together at all, and if so, how to characterize the curve $\gamma$ (respectively, the hypersurface $\Gamma$ ) along which this is possible. Answers to these questions will be provided by the junction conditions SSJC. Note that a timelike or spacelike curve in the two-dimensional base manifold $\left(\mathcal{B}, \boldsymbol{g}_{\mathcal{B}}\right)$ can be described simply by a function $R(\tau)$, where $R$ is the areal radius and $\tau$ the curve's arc-length which, in the timelike case, corresponds to the matching observer's proper time. The conditions $(i)$ and (iii) of the SSJC are then equivalent to the condition that the functional dependence $R(\tau)$ must be the same (up to a trivial translation in $\tau$ ) in both spacetimes to be matched.

\section{B. The Eisenstaedt theorem}

Perhaps the simplest attempt to model a compact body (star) in an expanding universe is trying to inglobate it in a FLRW spacetime and to assume, for simplicity, that the body is spherically symmetric. A direct consequence of the SSJC is the following intuitive appealing theorem due to Eisenstaedt (Eisenstaedt, 1977):

Theorem 2 (Eisenstaedt, 1977). Excise the full worldtube $\mathcal{W}_{r_{0}}$ of a comoving ball of comoving radius $r_{0}$ from a FLRW spacetime and insert instead a spherical symmetric inhomogeneity (hence a piece of a spherically symmetric spacetime together with a related matter model, satisfying Einstein's equations). Then a necessary condition for the resulting spacetime to satisfy Einstein's equation is that the MS energy of the inserted inhomogeneity equals that of the excised ball.

\footnotetext{
13 Recall that, because of our signature choice, the restriction of
} the metric to spacelike directions is negative definite. 
This says that the mean energy density (measured with the MS energy) of spherically-symmetric inhomogeneities must be the same as the one of the FLRW spacetime. That the Eisenstaedt Theorem is a consequence of the Darmois junction conditions was already pointed out in (Hartl, 2006).

\section{The Einstein-Straus vacuole revisited}

As another application of the above described matching procedure we revisit the Einstein-Straus solution (Einstein and Straus, 1945, 1946; Schücking, 1954), which originally consists on a Schwarzschild spacetime (called 'vacuole') matched to a dust FLRW universe with zero cosmological constant. Later, this model was generalized also to the case of a non-vanishing cosmological constant (Balbinot et al., 1988). We treat here the general case of an arbitrary cosmological constant and show that the SSJC allow substantial simplifications of the computations. This technique can also be applied to Bonnor's vacuole construction in LTB spacetimes.

Notice that the matching condition (56) implies, in particular, that the pressure must be continuous through the matching hypersurface. Since the interior is a vacuum spacetime, it follows that the pressure must vanishes also on the exterior part of the matching hypersurface and hence everywhere on the FLRW spacetime. That is why one has to restrict to dust FLRW spacetimes.

Since we leave the cosmological constant $\Lambda$ arbitrary (it may be positive, negative, or zero) the inner region is given, respectively, by a Schwarzschild-deSitter, Schwarzschild-anti-deSitter ${ }^{14}$, or Schwarzschild spacetime (all abbreviated henceforth by SdS). Recall that the SdS spacetime is given by the vacuum solution to Einstein equation with cosmological term

$$
\boldsymbol{g}^{\mathrm{SdS}}=V(R) \boldsymbol{d} T^{2}-V(R)^{-1} \boldsymbol{d} R^{2}-R^{2} \boldsymbol{g}_{S^{2}},
$$

where

$$
V(R)=1-\frac{2 m}{R}-\frac{\Lambda}{3} R^{2} .
$$

Above, $\boldsymbol{g}_{S^{2}}$ denotes the metric on the unit twosphere (31) and $m$ is a constant which represents the central mass.

A dust FLRW spacetime is given by the metric

$$
\boldsymbol{g}^{\mathrm{FLRW}}=\boldsymbol{d} t^{2}-a(t)^{2}\left(\frac{\boldsymbol{d} r^{2}}{1-k r^{2}}+r^{2} \boldsymbol{g}_{S^{2}}\right)
$$

14 The Schwarzschild-(anti-) de Sitter metric 59a is often called the Kottler solution, after Friedrich Kottler, who was the first to write down this metric in (Kottler, 1918). More details on its analytic and global structure may be found in (Gever, 1980). together with the matter energy-momentum tensor ${ }^{15}$ $\boldsymbol{T}=\varrho \underline{\boldsymbol{u}} \otimes \underline{\boldsymbol{u}}$, where $\boldsymbol{u}=\boldsymbol{\partial} / \boldsymbol{\partial} t$ is the (geodesic) velocity field of the cosmological dust and $\varrho$ is the matter energy density, which depends on $t$ only. Here $r$ is the comoving radial coordinate and $k$ is a constant which takes the values $0,-1,+1$, depending on whether the spatial slices have zero, negative, or positive curvature, respectively. The Einstein equation is then equivalent to the following set given by the Friedmann equation and a 'conservation equation':

$$
\begin{aligned}
& \left(\frac{\dot{a}}{a}\right)^{2}+\frac{k}{a^{2}}-\frac{C}{a^{3}}-\frac{\Lambda}{3}=0, \\
& \varrho a^{3}=\text { constant, }
\end{aligned}
$$

where the constant $C:=8 \pi \varrho_{0} a_{0}^{3} / 3$ depends on the initial conditions $a_{0}:=a\left(t_{0}\right)$ and $\varrho_{0}:=\varrho\left(t_{0}\right)$ at some 'initial' time $t_{0}$. Here, the dot denotes differentiation with respect to $t$ or, which is the same, along $\boldsymbol{u}$.

The central question is now the following: How shall we cut hypersurfaces $\Gamma_{\mathrm{SdS}}=\gamma_{\mathrm{SdS}} \times S^{2}$ and $\Gamma_{\mathrm{FLRW}}=\gamma_{\mathrm{FLRW}} \times$ $S^{2}$ in the spacetimes SdS and, respectively, FLRW in order that the resulting pieces can be matched? In order to apply the SSJC we have to compute the MS energy for both spacetimes. For the FLRW spacetime one has $R_{\mathrm{FLRW}}(t, r)=a(t) r$ and hence $\boldsymbol{d} R_{\mathrm{FLRW}}=\dot{a} r \boldsymbol{d} t+a \boldsymbol{d} r$ and $\langle\boldsymbol{d} R, \boldsymbol{d} R\rangle_{\mathrm{FLRW}}=g^{\mu \nu} R_{, \mu} R_{, \nu}=r^{2}\left(k+\dot{a}^{2}\right)-1$. From the definition (58) one then gets, using Friedmann's equation,

$$
E_{\mathrm{FLRW}}=\frac{4 \pi}{3} R_{\mathrm{FLRW}}^{3}\left(\varrho+\varrho_{\Lambda}\right),
$$

where $\varrho_{\Lambda}:=\Lambda / 8 \pi$ is the energy density associated with the cosmological constant. Notice that this expression, as well its derivation, is completely independent of the specific equation of state of the fluid and does not depend on the spatial curvature $k$. For the SdS case one has $R_{\mathrm{SdS}}=R$ and thus $\langle\boldsymbol{d} R, \boldsymbol{d} R\rangle_{\mathrm{SdS}}=-V(R)$ and

$$
E_{\mathrm{SdS}}=m+\frac{4 \pi}{3} R_{\mathrm{SdS}}^{3} \varrho_{\Lambda} .
$$

Now, the last two conditions of the SSJC, that is the continuity of areal radius and MS energy across the matching hypersurface (yet to be determined), are equivalent to the continuity of the areal radius $R_{\text {FLRW }}=$ $R_{\mathrm{SdS}}=: R$, together with the suggestive relation

$$
m=\frac{4 \pi}{3} R^{3} \varrho .
$$

This two conditions already determine the matching hypersurface. Indeed, inserting $R=R_{\mathrm{FLRW}}=a(t) r$ in (64)

15 Throughout we denote the metric-dual (1-form) of a vector $\boldsymbol{u}$ by underlining it, that is, $\underline{\boldsymbol{u}}:=\boldsymbol{g}(\boldsymbol{u}, \cdot)$ is the 1 -form metric-dual to vector $\boldsymbol{u}$. In local coordinates we have $\boldsymbol{u}=u^{\mu} \boldsymbol{\partial}_{\mu}$ and $\underline{\boldsymbol{u}}=$ $u_{\mu} \boldsymbol{d} x^{\mu}$, where $u_{\mu}:=g_{\mu \nu} u^{\nu}$. 
and using the relation $\varrho(t) a^{3}(t)=$ const. valid for dust FLRW models, one obtains the matching radius in terms of the FLRW comoving radial coordinate:

$$
r=r_{0}:=\left(\frac{m}{(4 \pi / 3) a_{0}^{3} \varrho_{0}}\right)^{1 / 3}=\text { constant } .
$$

Here $a_{0}:=a\left(t_{0}\right)$, and similarly for $\varrho$, where $t_{0}$ is some fixed 'initial' time. This means that the matching observer moves, in the FLRW spacetime, along the integral curve of $\boldsymbol{u}=\boldsymbol{\partial} / \boldsymbol{\partial} t$ with initial condition $\left(t_{0}, r_{0}\right)$ and hence is comoving with the cosmological matter.

So far we used the last two of the SSJC. As discussed above, the continuity of the areal radius and the arclength (the proper time, in the timelike case) of the matching curve are equivalent to the equality of the functional dependencies $R(\tau)$ (up to a possible trivial translation in $\tau$ ) which describe the matching curves in the two spacetimes to be matched. Now, because of (65), the matching curve (worldline) in the FLRW spacetime is simply

$$
R(\tau)=a(\tau) r_{0}
$$

where $a$ is the (unique) solution of the Friedmann equation 61a) with initial condition $a_{0}$ at $\tau_{0}=t_{0}$. (Recall that in FLRW the proper time of an observer moving along an integral line of $\boldsymbol{u}$ equals the cosmological time, hence $\tau=t$.) From what we said above, the same functional relation $R(\tau)$ must hold also in the SdSprovided we identify $R$ with the areal radius and $\tau$ with the matching observer's proper time, both referred to the SdS spacetime. This determines the matching curve in SdS.

Finally we need to show that the junction condition (ii) is satisfied, hence that the matching observer's accelerations coincide. Looking at the matching worldline from the FLRW spacetime, it is immediately clear that it is geodesic, hence its acceleration vanishes. To conclude the matching procedure, we just have to check that this is also true for the matching worldline in the SdS spacetime. For this, one has just to check that the function defined in (66) satisfies the geodesic equation for a radial motion. The latter is given by

$$
\dot{R}^{2}+V(R)=e^{2},
$$

where $e:=\boldsymbol{g}^{\mathrm{SdS}}(\boldsymbol{\partial} / \boldsymbol{\partial} T, \boldsymbol{v})=$ constant and $\boldsymbol{v}=\dot{T} \boldsymbol{\partial} / \boldsymbol{\partial} T+$ $\dot{R} \partial / \partial R$ is the matching observer in the SdS spacetime. (Equation (67) can be quickly derived from the fact that $\boldsymbol{v}\left(\boldsymbol{g}^{\mathrm{SdS}}(\boldsymbol{\partial} / \boldsymbol{\partial T}, \boldsymbol{v})\right)=0$, since $\boldsymbol{\partial} / \boldsymbol{\partial T}$ is Killing and $\boldsymbol{v}$ geodesic. Inserting $e:=\boldsymbol{g}^{\mathrm{SdS}}(\boldsymbol{\partial} / \boldsymbol{\partial} T, \boldsymbol{v})=V(R) \dot{T}$ in the normalization condition $1=\boldsymbol{g}^{\mathrm{SdS}}(\boldsymbol{v}, \boldsymbol{v})=V(R) \dot{T}^{2}-$ $\dot{R}^{2} / V(R)$ one arrives immediately at (67).) Now, inserting (66) with (65) in (67) and using the Friedmann equation (61a), one gets $\dot{R}^{2}+V(R)=1-k r_{0}^{2}$. Hence, the geodesic equation (67) is satisfied (with $e^{2}=1-k r_{0}^{2}$ ) and herewith all the four junction conditions.

\section{The McVittie model}

Among all models discussed in the literature which represent a quasi-isolated spherically-symmetric gravitating system melted into a cosmological spacetime, the one that is presumably best understood as regards its analytical structure as well as its physical assumptions is that of McVittie (McVittie, 1933), thanks to the careful analysis of Nolan (Nolan, 1998, 1999a b). Here we shall restrict to the 'flat' or $k=0$ model, which interpolates between an exterior Schwarzschild solution, describing a local mass, and a spatially flat (i.e. $k=0$ ) ambient FLRW universe. For simplicity we shall from now on refer to this model simply as the McVittie model. The cosmological constant is assumed to be zero, although this assumption is not essential (see the last paragraph of Section V.D.1).

This is not to say that this model is to be taken at face value in all its aspects. Its problems lie in the region very close to the central object, where the basic assumptions on the behavior of matter definitely turn unphysical. However, at radii much larger than (in geometric units) the central mass (to be defined below) the $k=0 \mathrm{McVittie}$ solution seems to provide a viable approximation for the transition between a homogeneous cosmological spacetime and a localized mass immersed in it. We will now briefly discuss this model and look at its geodesic equations, showing that they reduces to (12) in an appropriate weak-field and slow-motion limit. This provides another and more solid justification for the Newtonian approach we carried out in Section III.

The characterization of the McVittie model is made through two sets of a priori specifications. The first set concerns the metric (left side of Einstein's equations) and the second set the matter (right side of Einstein's equations). The former consists in an ansatz for the metric, which can formally be described as follows: Write down the Schwarzschild metric for the mass parameter $m$ in isotropic coordinates, add a conformal factor $a^{2}(t)$ to the spatial part, and allow the mass parameter $m$ to depend on time. Hence the metric reads

$$
\begin{aligned}
\boldsymbol{g} & =\left(\frac{1-m(t) / 2 r}{1+m(t) / 2 r}\right)^{2} \boldsymbol{d} t^{2} \\
& -\left(1+\frac{m(t)}{2 r}\right)^{4} a^{2}(t)\left(\boldsymbol{d} r^{2}+r^{2} \boldsymbol{g}_{S^{2}}\right),
\end{aligned}
$$

where $\boldsymbol{g}_{S^{2}}$ is given by (31). The metric (68) is obviously spherically symmetric with the spheres of constant radius $r$ being the orbits of the rotation group. We will discuss below what this ansatz actually entails. For later convenience we also introduce the orthonormal tetrad $\left\{\boldsymbol{e}_{\mu}\right\}_{\mu=0, \cdots, 3}$ with respect to (68), where $^{16}$

$$
\boldsymbol{e}_{\mu}:=\left\|\boldsymbol{\partial} / \boldsymbol{\partial} x^{\mu}\right\|^{-1} \boldsymbol{\partial} / \boldsymbol{\partial} x^{\mu}
$$

${ }^{16}$ We write $\|\boldsymbol{v}\|:=\sqrt{|\boldsymbol{g}(\boldsymbol{v}, \boldsymbol{v})|}$. 
and $\left\{x^{\mu}\right\}=\{t, r, \theta, \varphi\}$.

The second set of specifications, concerning the matter, is as follows: The matter is a perfect fluid with density $\varrho$ and isotropic pressure $p$. Hence its energy-momentum tensor is given by

$$
\boldsymbol{T}=\varrho \underline{\boldsymbol{u}} \otimes \underline{\boldsymbol{u}}+p(\underline{\boldsymbol{u}} \otimes \underline{\boldsymbol{u}}-\boldsymbol{g}) .
$$

Furthermore, and this is where the two sets of specifications make contact, the motion of the matter is given by

$$
\boldsymbol{u}=e_{0} .
$$

No further assumptions are made. In particular, an equation of state, like $p=p(\varrho)$, is not assumed. The reason for this will become clear soon.

Note that the vector field (71) is not geodesic for the metric (68) (unlike for the FLRW and Gautreau metrics), which immediately implies that the pressure cannot be constant. Being spherically symmetric, $\boldsymbol{u}$ is automatically vorticity free. The last property is manifest from its hypersurface orthogonality, which is immediate from (68). Moreover, $\boldsymbol{u}$ is also shear free. This, too, can be immediately read off (68) once one takes into account the result that for spherically symmetric metrics vanishing shear for a spherically symmetric vector field is equivalent to the corresponding spatial metric being conformally related to a spherically symmetric flat metric. This is obviously the case here.

The non-vanishing components of the Einstein tensor with respect to the orthonormal basis (69) are:

$$
\begin{aligned}
& \operatorname{Ein}\left(\boldsymbol{e}_{0}, \boldsymbol{e}_{0}\right)=3 F^{2}, \\
& \operatorname{Ein}\left(\boldsymbol{e}_{i}, \boldsymbol{e}_{j}\right)=-\left(3 F^{2}+2 \frac{A}{B} \dot{F}\right) \delta_{i j}, \\
& \operatorname{Ein}\left(\boldsymbol{e}_{0}, \boldsymbol{e}_{1}\right)=\frac{2}{R^{2}}\left(\frac{A}{B}\right)^{2}(a m)
\end{aligned}
$$

where an overdot denotes differentiation along $\boldsymbol{\partial} / \boldsymbol{\partial} t$. Before explaining the functions $A, B, R$, and $F$, we make the important observation that the Einstein tensor is spatially isotropic, where 'spatially' refers to the directions orthogonal to $\boldsymbol{e}_{0}$. By this we mean that $\operatorname{Ein}\left(\boldsymbol{e}_{i}, \boldsymbol{e}_{j}\right) \propto \delta_{i j}$ or, expressed more geometrically, that the spatial restriction of the Einstein tensor is proportional to the spatial restriction of the metric.

In (72) and in the following we set:

$$
A(t, r):=1+m(t) / 2 r, \quad B(t, r):=1-m(t) / 2 r,
$$

and

$$
R(t, r)=\left(1+\frac{m(t)}{2 r}\right)^{2} a(t) r
$$

where $R$ is the areal radius for the McVittie ansatz (68), and also

$$
F:=\frac{\dot{a}}{a}+\frac{1}{r B} \frac{(a m)^{\cdot}}{a} .
$$

In passing we note that $F$ has the geometric interpretation of being one third the expansion of the vector field $\boldsymbol{e}_{0}$, that is, $F=\operatorname{div}\left(\boldsymbol{e}_{0}\right) / 3$. Hence (72a) could also be written in the form $\operatorname{Ein}\left(\boldsymbol{e}_{0}, \boldsymbol{e}_{0}\right)=\left(\operatorname{div}\left(\boldsymbol{e}_{0}\right)\right)^{2} / 3$. We will see later that the product $a m$ which appears in (72C) also has a geometric meaning: it is just the Weyl part of the MS energy; see (81).

Now, the non-vanishing components of the energy momentum tensor (70) with (71) are:

$$
\boldsymbol{T}\left(\boldsymbol{e}_{0}, \boldsymbol{e}_{0}\right)=\varrho, \quad \boldsymbol{T}\left(\boldsymbol{e}_{i}, \boldsymbol{e}_{j}\right)=p \delta_{i j} .
$$

The $\left(\boldsymbol{e}_{0}, \boldsymbol{e}_{1}\right)$ component of Einstein's equation therefore implies $(a m)^{\circ}=0$, which means that the Weyl part of the MS energy is constant. Physically this can be interpreted as saying that the central object does not accrete any energy from the ambient matter. Using the constancy of $a m$ in (75) we immediately get:

$$
F=\frac{\dot{a}}{a}=: H
$$

Hence Einstein's equation is equivalent to the following three relations between the four functions $m(t), a(t), \varrho(t, r)$, and $p(t, r)$ :

$$
\begin{aligned}
& (a m)^{\cdot}=0, \\
& 8 \pi \varrho=3\left(\frac{\dot{a}}{a}\right)^{2}, \\
& 8 \pi p=-3\left(\frac{\dot{a}}{a}\right)^{2}-2\left(\frac{\dot{a}}{a}\right)^{\cdot}\left(\frac{1+m / 2 r}{1-m / 2 r}\right) .
\end{aligned}
$$

Note that here Einstein's equation has only three independent components (as opposed to four for a general spherically symmetric metric), which is a consequence of the fact, already stresses above, that the Einstein tensor for the McVittie ansatz (68) is spatially isotropic.

Equation (78a) can be immediately integrated:

$$
m(t)=\frac{m_{0}}{a(t)},
$$

where $m_{0}$ is an integration constant. Below we will show that this integration constant is to be interpreted as the mass of the central body. We will call the metric (68) together with condition (79) the McVittie metric.

Clearly the system (78) is under-determining. This is expected since no equation of state has yet been imposed. The reason why we did not impose such a condition can now be easily inferred from (78): whereas (78b) implies that $\varrho$ only depends on $t,(78 \mathrm{c})$ implies that $p$ depends on $t$ and $r$ iff $(\dot{a} / a)^{\dot{ }} \neq 0$. Hence a non-trivial relation $p=p(\varrho)$ is simply incompatible with the assumptions made so far. The only possible ways to specify $p$ are $p=0$ or $\varrho+p=0$. In the first case (78c) implies that $\dot{a} / a=0$ if $m_{0} \neq 0$ (since then the second term on the right-hand side is $r$ dependent, whereas the first is not, so that both must vanish separately), which corresponds to the exterior Schwarzschild solution, or $a(t) \propto t^{2 / 3}$ if 
$m_{0}=0$, which leads to the flat FLRW solution with dust. In the second case the fluid just acts like a cosmological constant $\Lambda=8 \pi \varrho$ (using the equation of state $\varrho+p=0$ in $\operatorname{div} \boldsymbol{T}=0$ it implies $\boldsymbol{d} p=0$ and this, in turn, using again the equation of state, implies $\boldsymbol{d} \varrho=0$ ) so that this case reduces to the Schwarzschild-deSitter solution. To see this explicitly, notice first that (78b/78c) imply the constancy of $H=\dot{a} / a=\sqrt{\Lambda / 3}$ and hence one has $a(t)=a_{0} \exp (t \sqrt{\Lambda / 3})$. With such a scalefactor the McVittie metric (68) with (79) turns into the Schwarzschild-deSitter metric (59) in disguise. The explicit formulae for the coordinate transformation relating the two can be found in Section 5 of (Robertson, 1928) and also in Section 7 of (Klioner and Soffel, 2005). Finally, note from (78a) that constancy of one of the functions $m$ and $a$ implies constancy of the other. In this case (78b $78 \mathrm{c})$ imply $\varrho=p=0$, so that we are dealing with the exterior Schwarzschild spacetime.

A specific McVittie solution can be obtained by choosing a function $a(t)$, corresponding to the scale function of the FLRW spacetime which the McVittie model is required to approach at spatial infinity, and the constant $m_{0}$, corresponding to the 'central mass'. Relations (78b/78c), and (79) are then used to determine $\varrho$, $p$, and $m$, respectively. Clearly this 'poor man's way' to solve Einstein's equation holds the danger of arriving at unrealistic spacetime dependent relations between $\varrho$ and $p$. This must be kept in mind when proceeding in this fashion. For further discussion of this point we refer to (Nolan, 1998, 1999a).

\section{Interpretation of the McVittie model}

In this section we discuss the interpretation of the McVittie model, its singularities, trapped regions, symmetry properties, and also the motion of the matter. In doing this, we shall take care to isolate those properties which are intrinsic to the ansatz (68) independent of the imposition of Einstein's equation. The analysis can then also be applied to all generalizations which maintain the ansatz (68). Generalizations in this sense have recently been discussed in (Faraoni and Jacques, 2007), on which we will comment at the end of this section.

According to what has just been said, we wish to regard the McVittie solution as a candidate model for an isolated mass $m_{0}$ in an 'otherwise' flat FLRW universe with scale function $a(t)$. As already emphasized in the introduction, this requires specific justification in view of the fact that simple superpositions of solutions are disallowed by the nonlinearities. A set of criteria for when a solution represents a localized mass immersed in a flat FLRW background have been proposed and discussed in detail in (Nolan, 1998). The basic idea is to employ the MS energy (in a spherically symmetric context, where it is equivalent to the Hawking mass) in order to detect localized sources of gravity. We will follow this approach and for this purpose we compute the Ricci and the Weyl part of the MS energy.

This we now do for the class of metrics (68), without at first making any use of Einstein's equation. The geometric definition of the MS energy in terms of the sectional curvature, together with formula A10b for its Ricci part specialized to metrics with spatially isotropic Einstein tensor, implies that the Ricci part of the MS energy is given by

$$
E_{\mathrm{R}}=\frac{1}{6} R^{3} \operatorname{Ein}\left(e_{0}, e_{0}\right) .
$$

The Weyl part is then obtained as the difference between the full MS energy and (80). We use the expression (58) for the former and write $\langle\boldsymbol{d} R, \boldsymbol{d} R\rangle=\left(\boldsymbol{e}_{0}(R)\right)^{2}-\left(\boldsymbol{e}_{1}(R)\right)^{2}$. The part involving $\boldsymbol{e}_{0}(R)$ equals (80), due to the relation $\operatorname{Ein}\left(\boldsymbol{e}_{0}, \boldsymbol{e}_{0}\right)=3\left(\boldsymbol{d} R\left(\boldsymbol{e}_{0}\right) / R\right)^{2}$, which, e.g., follows from the comment below Eq. (75) and (D54) (for vanishing shear). The Weyl part of the MS energy is therefore given by $(R / 2)\left(1-\left(\boldsymbol{e}_{1}(R)\right)^{2}\right)$. From (74) we calculate $\boldsymbol{e}_{1}(R)$ and hence obtain for the Weyl part of the MS energy:

$$
E_{\mathrm{w}}=a m \text {. }
$$

Now we invoke Einstein's equation with source (70) and four-velocity (71). Then the Ricci and Weyl contributions to the MS energy can be written in the following form, also taking into account (79),

$$
\begin{aligned}
& E_{\mathrm{R}}=\frac{4 \pi}{3} R^{3} \varrho, \\
& E_{\mathrm{W}}=m_{0} .
\end{aligned}
$$

Identifying the gravitational mass of the central object with the Weyl part of the MS energy, its constancy means that no energy is accreted from the ambient matter. As regards the Ricci part, note that the factor $(4 \pi / 3) R^{3}$ in (82a) is smaller than the proper geometric volume within the sphere of areal radius $R$. This can be attributed to the gravitational binding energy that diminishes the gravitational mass of a lump of matter below the value given by the proper space integral of $\boldsymbol{T}\left(\boldsymbol{e}_{0}, \boldsymbol{e}_{0}\right)$. This is shown in more detail in Appendix D.4, in particular in the exact equation (D47) and its leading order approximation (D49).

It is also important to note that the central gravitational mass in McVittie's spacetime may be modeled by a shear-free perfect-fluid star of positive homogeneous energy density (Nolan, 1993). The matching is performed along a world-tube comoving with the cosmological fluid, across which the energy density jumps discontinuously. This means that the star's surface is comoving with the cosmological fluid and hence, in view of (89), that it geometrically expands (or contracts). This feature, however, should be merely seen as an artifact of the McVittie model (in which the relation (89) holds), rather than a general property of compact objects in any cosmological spacetimes. Positive pressure within the star seems to be only possible if $2 a \ddot{a}+\dot{a}^{2}<0$ (see Eq. (3.27) in (Nolan, 1993) with $a=\exp (\beta / 2)$ ), that is, for deceleration parameters $q>1 / 2$. 
Next we comment on the singularity properties of the McVittie model. From (78c) it is clear that, unless $\dot{a} / a$ is constant (the Schwarzschild-deSitter case) or $m=0$ (FLRW case), the pressure diverges at $r=m / 2$ (that is at $R=2 m_{0}=R_{S}$ ). In fact, this corresponds to a genuine curvature singularity which is built into the McVittie ansatz (68) independently of any further assumption. To see that $r=m / 2$ (corresponding to $R=2 a m=2 E_{\mathrm{W}}$ ) is a singularity it suffices to consider the scalar curvature (i.e. the Ricci scalar) of (68),

$$
\text { Scal }=-12 F^{2}-6 \frac{A}{B} \dot{F},
$$

which is readily computed from (72). In (Carrera and Giulini, 2009) we show that this becomes singular in the limit $r \rightarrow m / 2$, with the only exceptions being the following three special cases: ( $i) m=0$ and $a$ arbitrary (FLRW spacetimes), (ii) $a$ and $m$ constant (Schwarzschild spacetime), and (iii) $(a m)^{\cdot}=0$ and $(\dot{a} / a)^{\cdot}=0$ (Schwarzschild-de Sitter spacetime). This means that, as long as we stick to the ansatz (68), at $r=m / 2$ there will always (with the only exceptions listed above) be a singularity in the Ricci part of the curvature and thus, assuming Einstein's equation is satisfied, also in the energy momentum tensor, irrespectively of the details of its underlying matter model. Hence any attempt to eliminate this singularity by maintaining the ansatz (68) and merely modifying the matter model is doomed to fail. In particular, this is true for the generalizations presented in (Faraoni and Jacques, 2007), contrary to what is claimed in that work. We also remark that it makes no sense to absorb the singular factors $1 / B$ in front of the time derivatives by writing $(A / B) \boldsymbol{\partial} / \boldsymbol{\partial} t$ as $\boldsymbol{e}_{0}$ and then argue, as was done in (Faraoni and Jacques, 2007), that this eliminates the singularity. The point is simply that then $e_{0}$ applied to any continuously differentiable function diverges as $r \rightarrow m / 2$. Below we will show that this singularity lies within a trapped region. Turning back to the McVittie model, recall that in this case it is assumed that the fluid moves along the integral curves of $\boldsymbol{\partial} / \boldsymbol{\partial} t$, which become lightlike in the limit as $r$ tends to $m / 2$. Their acceleration is given by the gradient of the pressure, which necessarily diverges in the limit $r \rightarrow m / 2$, as one explicitly sees from (91). For a more detailed study of the geometric singularity at $r=m / 2$, see (Nolan, 1999a, b).

For spherically symmetric spacetimes the Weyl part of the curvature has only a single independent component (see (D8a)) which, by its very definition, is $-2 / R^{3}$ times the Weyl part of the MS energy (see (D36)). The square of the Weyl tensor for the ansatz (68) may then be conveniently expressed as (see (81) and (D37))

$$
\langle\text { Weyl, Weyl }\rangle=48 \frac{(a m)^{2}}{R^{6}} .
$$

This shows that $R=0$ also corresponds to a genuine curvature singularity, though this is not part of the region covered by our original coordinate system, for which $r>$ $m / 2$ (that is $R>2 E_{\mathrm{W}}$ ).

It is instructive to also determine the trapped regions of McVittie spacetime. We do this just using the McVittie ansatz (68) and making no further assumptions. Recall that a spacelike two-sphere $S$ is said to be trapped, marginally trapped, or untrapped if the product $\theta^{+} \theta^{-}$of the expansions (defined below Eq. (D29)) for the ingoing and outgoing future-pointing null vector fields normal to $S$ is positive, zero, or negative. Taking $S$ to be $S_{R}$, that is, a sphere of symmetry with areal radius $R$, it immediately follows from (D31) that $S_{R}$ is trapped, marginally trapped, or untrapped iff $\langle\boldsymbol{d} R, \boldsymbol{d} R\rangle$ is positive, zero, or negative, respectively. This corresponds to timelike, lightlike, or spacelike $\boldsymbol{d} R$, or, equivalently, in in view of (58), to $2 E-R$ being positive, zero, or negative, respectively. Using (80) together with (72a), the MS energy for the McVittie ansatz can be written as $E=E_{\mathrm{w}}+R^{3} F^{2} / 2$, so that

$$
2 E-R=F^{2} R^{3}-R+R_{S},
$$

where $R_{S}:=2 E_{\mathrm{W}}$ denotes the 'generalized' Schwarzschild radius. Note that in general $F$ depends itself on the radial coordinate - except for the McVittie case, in which one has $F=H=: 1 / R_{H}\left(R_{H}\right.$ denotes the Hubble radius). For computational simplicity we specialize in the following to the McVittie case, referring to (Carrera and Giulini, 2009) for the general case. Doing this, (85) becomes a cubic polynomial in $R$ which is positive for $R=0$ and tends to $\pm \infty$ for $R \rightarrow \pm \infty$. Hence it always has a negative zero (which does not interest us) and two positive zeros iff

$$
R_{S} / R_{H}<2 / 3 \sqrt{3} \approx 0.38 .
$$

This clearly corresponds to the physical relevant case where the Schwarzschild radius is much smaller than the Hubble radius. One zero lies in the vicinity of the Schwarzschild radius and one in the vicinity of the Hubble radius, corresponding to two marginally trapped spheres. The exact expressions for the zeros can be easily written down, but are not very illuminating. In leading order in the small parameter $R_{S} / R_{H}$, they are approximated by

$$
\begin{aligned}
& R_{1} \approx R_{S}\left(1+\left(R_{S} / R_{H}\right)^{2}\right), \\
& R_{2} \approx R_{H}\left(1-R_{S} / 2 R_{H}\right) .
\end{aligned}
$$

From this one sees that for the McVittie model the radius of the marginally trapped sphere of Schwarzschild spacetime $\left(R_{S}\right)$ increases and that of the FLRW spacetime $\left(R_{H}\right)$ decreases. The first feature can be understood as an effect of the presence of cosmological matter, whereas the latter is an effect of the presence of a central mass abundance. All the spheres with $R<R_{1}$ or $R>R_{2}$ are trapped and those with $R_{1}<R<R_{2}$ are untrapped. In particular, the singularity $r=m / 2$, that is $R=2 E_{\mathrm{W}}=R_{S}$, lies within the inner trapped region. 
Another aspect concerns the global behavior of the McVittie ansatz (68). We note that each hypersurface of constant time $t$ is a complete Riemannian manifold, which, besides the rotational symmetry, admits a discrete isometry given in $(r, \theta, \varphi)$ coordinates by

$$
\phi(r, \theta, \varphi)=\left((m / 2)^{2} r^{-1}, \theta, \varphi\right) .
$$

This corresponds to an inversion at the two-sphere $r=$ $m / 2$ and shows that the hypersurfaces of constant $t$ can be thought of as two isometric asymptotically-flat pieces joined together at the totally geodesic (being a fixedpoint set of an isometry and hence also minimal) twosphere $r=m / 2$. Except for the time-dependent factor $m(t)$, this is analogous to the geometry of the $t=$ const. slices in the Schwarzschild metric (the difference being that (88) does not extend to an isometry of the spacetime metric unless $\dot{m}=0$ ). This means, in particular, that the McVittie metric cannot literally be interpreted as corresponding to a point particle sitting at $r=0(r=0$ is at infinite metric distance $)$ in a flat FLRW universe, just like the Schwarzschild metric does not correspond to a point particle sitting at $r=0$ in Minkowski space. Unfortunately, McVittie seems to have interpreted his solution in this fashion (McVittie, 1933) which even until recently gave rise to some confusion in the literature (e.g. (Ferraris et al., 1996; Gautreau, 1984; Sussman, 1988)). A clarification was given by (Nolan, 1999a).

We now briefly discuss the basic properties of the motion of cosmological matter for the McVittie model. We already mentioned that the vorticity and shear of the four-velocity $\boldsymbol{u}$ vanish identically. On the other hand, the expansion (divergence of $\boldsymbol{u}$ ) is

$$
\theta=3 H,
$$

just as in the FLRW case (recall that here $H:=\dot{a} / a$ is defined as in the FLRW case, see (77)). In particular, the expansion of the cosmological fluid is homogeneous in space. The expression for the variation of the areal radius along the integral lines of $\boldsymbol{u}$ (that is the velocity of cosmological matter measured in terms of its proper time and the areal radius) is also just as in the FLRW case:

$$
\boldsymbol{u}(R)=H R,
$$

which is nothing but Hubble's law. The acceleration of $\boldsymbol{u}$, which in contrast to the FLRW case does not vanish, is given by

$$
\boldsymbol{\nabla}_{\boldsymbol{u}} \boldsymbol{u}=\frac{m_{0}}{R^{2}}\left(\frac{1+m / 2 r}{1-m / 2 r}\right) \boldsymbol{e}_{1}
$$

In leading order in $m_{0} / R$ this corresponds to the acceleration of the observers moving along $\boldsymbol{\partial} / \boldsymbol{\partial} T$ in Schwarzschild spacetime (see Eq. (132) with $\Lambda=0$ ).

We conclude this subsection by commenting on the attempts to generalize the McVittie model. The first obvious generalization consists in allowing a non-vanishing cosmological constant. This is however trivial, since it is equivalent to the substitution $\varrho \rightarrow \varrho+\varrho_{\Lambda}$ and $p \rightarrow p+p_{\Lambda}$ in (78), where $\varrho_{\Lambda}:=\Lambda / 8 \pi$ and $p_{\Lambda}:=-\Lambda / 8 \pi$ are, respectively, the energy-density and pressure associated to the cosmological constant $\Lambda$. In (Faraoni and Jacques, 2007), attempts to generalize the McVittie model have focused on keeping the ansatz (68) and relaxing the conditions on the matter in various ways. More precisely, they show that the McVittie case may be generalized to allow for radial fluid motions relative to the $\boldsymbol{e}_{0}$ observer field (that is relaxing condition (71)), provided one also allows for a non-vanishing radial heat flow. Both energy flows are necessary in order to get new solutions consistent with the ansatz (68), even though the two radial energy flows do not cancel in the energy balance. As a result, the Weyl part of the MS energy will now change in time so that the new solutions correspond to inhomogeneities of variable strength due to accretion or loss of energy. For further analysis of these solutions we refer to (Carrera and Giulini, 2009). Another generalization of the McVittie model, this time away from the ansatz (68), is given in (Sultana and Dyer, 2005). It is constructed by applying a particular (time-dependent) conformal transformation to the Schwarzschild spacetime. As we will show in (Carrera and Giulini, 2009), the metric so obtained cannot be written in the McVittie form (68), contrary to what is suggested in Faraoni and Jacques, 2007). The corresponding energy-momentum tensor (obtained via Einstein's equation) can be interpreted as a sum of two contributions, one due to a perfect fluid and the other to a null fluid.

\section{Motion of a test particle in McVittie spacetime}

We are interested in the motion of a test particle (idealizing a planet or a spacecraft) in McVittie's spacetime. In (McVittie, 1933) it was concluded within a slow-motion and weak-field approximation that Keplerian orbits do not expand as measured with the 'cosmological geodesic radius' $r_{*}=a(t) r$. Later (Pachner, 1963) and (Noerdlinger and Petrosian, 1971) argued for the presence of the acceleration term (77) proportional to $\ddot{a} / a$ within this approximation scheme, hence arriving at (12a). In the following we shall show how to arrive at (12a) from the exact geodesic equation of the McVittie metric by making clear the approximations involved. Related recent discussions were given in (Bolen et al., 2001), where the effects of cosmological expansion on the periastron precession and eccentricity are discussed for constant Hubble parameter $H:=\dot{a} / a$.

We will again work with the areal radius $R$. Note that for fixed $t$ the map $r \mapsto R(t, r)$ is 2-to- 1 and that $R \geq 2 m_{0}$, where $R=2 m_{0}$ corresponds to $r=m_{0} / 2 a$. Hence we restrict the coordinate transformation (74) to the region $r>m_{0} / 2 a$ where it becomes a diffeomorphism onto the region $R>2 m_{0}$. (The region $R<2 m_{0}$ was investigated in (Nolan, 1999b).) Reintroducing factors of 
$c$, McVittie's metric assumes the (non-diagonal) form in the region $R>2 m_{0}$ (i.e. $r>m_{0} / 2 a(t)$ )

$$
\begin{aligned}
\boldsymbol{g}= & \left(1-2 \mu(R)-h(t, R)^{2}\right) c^{2} \boldsymbol{d} t^{2} \\
& +\frac{2 h(t, R)}{\sqrt{1-2 \mu(R)}} c \boldsymbol{d} t \boldsymbol{d} R-\frac{d R^{2}}{1-2 \mu(R)}-R^{2} \boldsymbol{g}_{S^{2}},
\end{aligned}
$$

where we put

$$
\mu(R):=\frac{m_{0}}{R}, \quad h(t, R):=\frac{H(t) R}{c}
$$

with $H(t):=(\dot{a} / a)(t)$, as usual.

The equations for a timelike geodesic (i.e. parametrized with respect to proper time), $\tau \mapsto z^{\mu}(\tau)$ with $g(\dot{z}, \dot{z})=$ $c^{2}$, follows via variational principle from the Lagrangian $\mathcal{L}(z, \dot{z})=(1 / 2) g_{\mu \nu}(z) \dot{z}^{\mu} \dot{z}^{\nu}$. Spherical symmetry implies conservation of angular momentum. Hence we may choose the particle's orbit to lie in the equatorial plane $\theta=\pi / 2$. The constant modulus of angular momentum is

$$
R^{2} \dot{\varphi}=L
$$

The remaining two equations are then coupled secondorder ODEs for $t(\tau)$ and $R(\tau)$. However, we may replace the first one by its first integral that results from $\boldsymbol{g}(\dot{\boldsymbol{z}}, \dot{\boldsymbol{z}})=c^{2}$ :

$$
\begin{aligned}
& \left(1-2 \mu(R)-h^{2}(t, R)\right) c^{2} \dot{t}^{2} \\
& +\frac{2 h(t, R)}{\sqrt{1-2 \mu(R)}} c \dot{t} \dot{R}-\frac{\dot{R}^{2}}{1-2 \mu(R)}-(L / R)^{2}=c^{2} .
\end{aligned}
$$

The remaining radial equation is given by

$$
\begin{aligned}
\ddot{R} & -\left(1-2 \mu(R)-h^{2}(t, R)\right) \frac{L^{2}}{R^{3}} \\
+ & \frac{m_{0} c^{2}}{R^{2}}(1-2 \mu(R)) \dot{t}^{2} \\
- & R\left(\dot{H}(t)(1-2 \mu(R))^{1 / 2}\right. \\
& \left.\quad+H(t)^{2}\left(1-\mu(R)-h^{2}(t, R)\right)\right) \dot{t}^{2} \\
& -\frac{\left(\mu(R)-h^{2}(t, R)\right)}{1-2 \mu(R)} \frac{\dot{R}^{2}}{R} \\
+ & \frac{2\left(\mu(R)-h^{2}(t, R)\right)}{\sqrt{1-2 \mu(R)}} c H(t)(\dot{R} / c) \dot{t}=0 .
\end{aligned}
$$

Recall that $m_{0}=G M / c^{2}$, where $M$ is the mass of the central star in standard units.

Equations (95|96) are exact. We are interested in orbits of slow-motion (compared with the speed of light) in the region where

$$
R_{S} \ll R \ll R_{H} .
$$

Recall that $R_{S}$ and $R_{H}$ are the Schwarzschild and the Hubble radius, respectively (see (5),(6) ). The latter condition clearly covers all situations of practical applicability in the Solar System, since the Schwarzschild radius
$R_{S}$ of the Sun is about $3 \mathrm{~km}=2 \cdot 10^{-8} \mathrm{AU}$ and the Hubble radius $R_{H}$ is about $13.7 \cdot 10^{9} \mathrm{ly}=8.7 \cdot 10^{14} \mathrm{AU}$.

The approximation now consists in considering small perturbations of Keplerian orbits. Let $T$ be a typical time scale of the problem, like the period for closed orbits or else $R / v$ with $v$ a typical velocity. The expansion is then with respect to the following two parameters:

$$
\varepsilon_{1} \approx \frac{v}{c} \approx\left(\frac{m_{0}}{R}\right)^{\frac{1}{2}}
$$

corresponding to a slow-motion and weak-field approximation, and

$$
\varepsilon_{2} \approx H T
$$

corresponding to the approximation for small ratios of characteristic-times to the age of the universe. In order to make the expression to be approximated dimensionless, we multiply (95) by $1 / c^{2}$ and (96) by $T^{2} / R$. Then we expand the right hand sides in powers of the parameters (98), using the fact that $h:=(H R / c) \approx \varepsilon_{1} \varepsilon_{2}$. From this and (94) we obtain (12) if we keep only terms to zeroorder in $\varepsilon_{1}$ and leading (i.e. quadratic) order in $\varepsilon_{2}$, where we also re-express $R$ as function of $t$. Note that in this approximation the areal radius $R$ is equal to the spatial geodesic distance on the $t=$ const. hypersurfaces.

We already mentioned that in the special case of constant $H=\dot{a} / a$ the McVittie solution turns into the Schwarzschild-deSitter metric (59), which also describes the spacetime inside the Einstein-Straus vacuoles in case of non-vanishing cosmological constant. Recently, exact expressions in terms of hyperelliptic integrals for the integrated geodesic equation in Schwarzschild-deSitter spacetimes where derived in (Hackmann and Lämmerzahl, 2008a b). Moreover, a general discussion of Solar-System effects in Schwarzschild-deSitter, like gravitational redshift, light deflection, time delay, perihelion precession, geodetic precession, and effects on Doppler tracking, has been given in (Kagramanova et al., 2006). For example, it was found that a non-vanishing $\Lambda$ could account for the anomalous Pioneer acceleration if its value was $-10^{-37} \mathrm{~m}^{-2}$, which is minus $10^{15}$ times the current most probable value. That value would also give rise to a perihelion precession four orders of magnitude larger than the accuracy to which this effect has been measured today.

We conclude by commenting on the geodesic equation in the generalizations of McVittie's model given in (Faraoni and Jacques, 2007) and in (Sultana and Dyer, 2005). An essential feature which distinguish these solutions from the McVittie one, is that in the former the Weyl part of the MS energy $E_{\mathrm{W}}=a m$ is not a constant as for McVittie but varies in time, meaning that there is an accretion of cosmological matter by the inhomogeneity (see (Carrera and Giulini, 2009)). In view of the fact that the combination

$$
m / r=A^{2} E_{\mathrm{w}} / R \approx E_{\mathrm{w}} / R
$$


contained in the McVittie ansatz gives (minus) the 'Newtonian' part of the potential in the slow-motion and weakfield approximation (see Section V.D.2), we deduce that in order to get the geodesic equation for the generalized McVittie models it suffices to substitute $m_{0}$ with $E_{\mathrm{W}}$ in the equation of motion derived in Section V.D.2. This means that the strength of the central attraction varies in time, leading to an in- or out-spiraling of the orbits if $E_{\mathrm{W}}$ is increasing or decreasing, respectively.

\section{Exact condition for non-expanding circular orbits in McVittie spacetime}

Analogously to Section IV.B. where we ask whether there exist non-expanding circular orbits (i.e. of constant areal radius) of the electron-proton system in a FLRW spacetime, we now ask whether there exist nonexpanding circular orbits of an (uncharged) test particle around the central mass. The necessary and sufficient condition for this to happen follows from inserting $R=$ const in the radial part of the geodesic equation (96) and using the normalization condition (95) of the four-velocity in order to eliminate $\dot{t}$. In terms of the dimensionless quantities $h(t):=R H(t) / c, l:=L / R c$, and $\mu:=m_{0} / R$, the condition for the existence of nonexpanding circular orbits can be given the following form:

$$
\frac{R}{c} \dot{h}=\frac{\left(1-2 \mu-h^{2}\right)\left(\mu\left(1+3 l^{2}\right)-l^{2}-h^{2}\right)}{\left(1+l^{2}\right) \sqrt{1-2 \mu}} .
$$

As for the electron-proton system in an FLRW spacetime (see (51)) this is a first-order autonomous ODE for $h(t)$ and therefore the Hubble function. In the present case the ODE is even simpler since it has the elementary form $\dot{h}=p\left(h^{2}\right)$, where $p$ is a polynomial of degree two with constant coefficients. From (100), to leading order in the small quantities $\mu, l^{2}$, and $h^{2}$, we get the same approximate ODE (54) and hence the same approximate solutions (55). Hence, the same conclusions as drawn for the electron-proton system in FLRW apply here.

From (100) it follows that stationary solutions $h(t)=$ const $=: h_{0}$, corresponding to an exponentially-growing scale factor (52) (and hence leading to a Schwarzschildde Sitter spacetime), are those where $h_{0}$ satisfies

$$
\frac{l^{2}+h_{0}^{2}}{\left(1+3 l^{2}\right)}=\mu
$$

where we used that the first factor on the numerator of the right-hand side of (100) is nonzero, as can be immediately inferred from the normalization condition (95). Notice that for a vanishing Hubble parameter (that is for $\left.h_{0}=0\right)$ the above condition reduces to the third Kepler law in Schwarzschild spacetime, as expected. The effect of a non-vanishing Hubble parameter is again that we must provide the orbiting particle with a smaller angular velocity (smaller $l$ ) in order to keep it on a non-expanding circular orbit with the same radius. The largest radius at which in a McVittie spacetime with exponentiallyexpanding scale factor (that is a Schwarzschild-de Sitter spacetime) there is a non-expanding circular orbit follows from (101) in the limit $l \rightarrow 0$. Then the condition reduces to $h_{0}^{2}=\mu$ which, solving for $R$, gives $\left(R_{S} R_{H}^{2} / 2\right)^{1 / 3}$. This, exactly corresponds to the critical radius (16), taking into account that $q_{0}=-1$ for an exponentially-growing scale factor.

\section{KINEMATICAL EFFECTS}

In this section we discuss the influence of cosmic expansion upon measurements of relative distances, velocities, and accelerations. These kinematical notions loose their a priori meaning in general spacetimes, in particular in time-dependent ones. Hence it is of utmost importance to carefully reconsider statements concerning such notions and their precise relations to locally observable quantities.

\section{A. Einstein- versus cosmological simultaneity}

Misidentifications in the notion of simultaneity can give rise to apparent anomalies in velocities and acceleration. Such an effect has e.g. been suggested in (Rosales and Sanchez-Gomez, 1998) and again in (Rosales, 2002) to be able to account for the PA. Their argument says that in a spatially flat FLRW universe the mismatch between adapted cosmological coordinates on the one hand and radar coordinates on the other just amount to an apparent difference in radial acceleration of magnitude (11). We agree on the existence and conceptual importance of such an effect but we disagree on the magnitude, which seems to have been grossly overestimated as we will show below.

The cause of such effects lies in the way one actually measures spatial distances and determines the clock readings they are functions of (a trajectory is a 'distance' for each given 'time'). The point is this: equations of motions give us, for example, simultaneous (with respect to cosmological time) spatial geodesic distances as functions of cosmological time. This is what we implicitly did in the Newtonian analysis. But, in fact, spacecraft ranging is done by exchanging electromagnetic signals. The notions of spatial distance and simultaneity thereby implicitly used are not the same as those we referred to above. Hence the analytical expression of the 'trajectory' so measured will be different.

We first recall the local version of Einstein simultaneity in general spacetimes $(\mathcal{M}, \boldsymbol{g})$. We take $d s=g_{\mu \nu} d x^{\mu} d x^{\nu}$ to carry the unit of length so that $d \tau=d s / c$ carries the unit of time. In general coordinates $\left\{x^{\mu}\right\}=\left\{t, x^{i}\right\}$, where $x^{0}=t$ denotes the timelike coordinate, the metric reads

$$
d s^{2}=g_{\mu \nu} d x^{\mu} d x^{\nu}=g_{t t} d t^{2}+2 g_{t i} d t d x^{i}+g_{i j} d x^{i} d x^{j} .
$$


The observer at fixed spatial coordinates is given by the vector field (normalized to $\boldsymbol{g}(\boldsymbol{u}, \boldsymbol{u})=c^{2}$ )

$$
\boldsymbol{u}=c\|\boldsymbol{\partial} / \boldsymbol{\partial} t\|^{-1} \boldsymbol{\partial} / \boldsymbol{\partial} t=\frac{c}{\sqrt{g_{t t}}} \boldsymbol{\partial} / \boldsymbol{\partial} t
$$

Consider the light cone with vertex $p \in \mathcal{M}$; one has $d s^{2}=$ 0 , which allows to solve for $d t$ in terms of the $d x^{i}$ (all functions $g_{\mu \nu}$ are evaluated at $p$, unless noted otherwise):

$$
d t_{1,2}=-\frac{g_{t i}}{g_{t t}} d x^{i} \pm \sqrt{\left(\frac{g_{t i} g_{t j}}{g_{t t}^{2}}-\frac{g_{i j}}{g_{t t}}\right) d x^{i} d x^{j}}
$$

The plus sign corresponds to the future light-cone at $p$, the negative sign to the past light cone. An integral line of $\boldsymbol{u}$ in a neighborhood of $p$ cuts the light cone in two points, $q_{+}$and $q_{-}$. If $t_{p}$ is the time assigned to $p$, then $t_{q_{+}}=t_{p}+d t_{1}$ and $t_{q_{-}}=t_{p}+d t_{2}$. The coordinate-time separation between these two cuts is $t_{q_{+}}-t_{q_{-}}=d t_{1}-d t_{2}$, corresponding to a proper time $\sqrt{g_{t t}}\left(d t_{1}-d t_{2}\right) / c$ for the observer $\boldsymbol{u}$. This observer will associate a radar-distance $d l_{*}$ to the event $p$ of $c / 2$ times that proper time interval, that is:

$$
d l_{*}^{2}=h=\left(\frac{g_{t i} g_{t j}}{g_{t t}}-g_{i j}\right) d x^{i} d x^{j} .
$$

The event on the integral line of $\boldsymbol{u}$ that the observer will call Einstein-synchronous with $p$ lies in the middle between $q_{+}$and $q_{-}$. Its time coordinate is in first-order approximation given by $\frac{1}{2}\left(t_{q_{+}}+t_{q_{-}}\right)=t_{p}+\frac{1}{2}\left(d t_{1}+d t_{2}\right)=$ $t_{p}+d t$, where

$$
d t:=\frac{1}{2}\left(d t_{1}+d t_{2}\right)=-\frac{g_{t i}}{g_{t t}} d x^{i}
$$

This means the following: The integral lines of $\boldsymbol{u}$ are parametrized by the spatial coordinates $\left\{x^{i}\right\}_{i=1,2,3}$. Given a point $p$, specified by the orbit-coordinates $x_{p}^{i}$ and the time-coordinate $t_{p}$, we consider a neighboring orbit of $\boldsymbol{u}$ with orbit-coordinates $x_{p}^{i}+d x^{i}$. The event on the latter which is Einstein synchronous with $p$ has a time coordinate $t_{p}+d t$, where $d t$ is given by (106), or equivalently

$$
\theta:=d t+\frac{g_{t i}}{g_{t t}} d x^{i}=0
$$

Using a differential geometric language we may say that Einstein simultaneity defines a distribution $\theta=0$.

The metric (102) can be written in terms of the radardistance metric $h$ (105) and the simultaneity 1-form $\theta$ as follows:

$$
d s^{2}=g_{\mu \nu} d x^{\mu} d x^{\nu}=g_{t t} \theta^{2}-h,
$$

showing that the radar-distance is just the same as the Einstein-simultaneous distance. A curve $\gamma$ in $\mathcal{M}$ intersects the flow lines of $\boldsymbol{u}$ perpendicularly iff $\theta(\dot{\gamma})=0$, which is just the condition that neighboring clocks along $\gamma$ are Einstein synchronized.
We now apply the foregoing to isotropic cosmological metrics. In what follows we drop for simplicity the angular dimensions. Hence we consider metrics of the form

$$
d s^{2}=c^{2} d t^{2}-a(t)^{2} d r^{2} .
$$

The comoving observer field,

$$
\boldsymbol{u}=c \boldsymbol{\partial} / \partial t
$$

is geodesic and of expansion $3 H$. On a hypersurface of constant $t$ the radial geodesic distance is given by $a(t) r$. Making this distance into a spatial coordinate, $r_{*}$, we consider the coordinate transformation

$$
t \mapsto t_{*}:=t, \quad r \mapsto r_{*}:=a(t) r
$$

The field $\boldsymbol{\partial} / \partial t_{*}$ is given by

$$
\partial / \partial t_{*}=\partial / \partial t-H r \partial / \partial r
$$

to which the observer field,

$$
\boldsymbol{u}_{*}:=c\left\|\boldsymbol{\partial} / \partial t_{*}\right\|^{-1} \boldsymbol{\partial} / \partial t_{*}
$$

corresponds. In contrast to (110), whose flow connects comoving points of constant coordinate $r$, the flow of (112) connects points of constant geodesic distances, as measured in the surfaces of constant cosmological time. This could be called cosmologically instantaneous geodesic distance. It is now very important to realize that this notion of distance is not the same as the radar distance that one determines by exchanging light signals in the usual (Einsteinian) way. Let us explain this in detail:

From (111) we have $a d r=d r_{*}-r_{*} H d t$, where $H:=$ $\dot{a} / a$ (Hubble parameter). Rewriting the metric (109) in terms of $t_{*}$ and $r_{*}$ yields

$$
\begin{aligned}
d s^{2} & =c^{2}\left(1-\left(H r_{*} / c\right)^{2}\right) d t_{*}^{2}-d r_{*}^{2}+2 H r_{*} d t d r_{*} \\
& =\underbrace{c^{2}\left\{1-\left(H r_{*} / c\right)^{2}\right\}}_{g_{t_{*} t_{*}}}\{\underbrace{d t_{*}+\frac{H r_{*} / c^{2}}{1-\left(H r_{*} / c\right)^{2}} d r_{*}}_{\theta}\}^{2} \\
& -\underbrace{\frac{d r_{*}^{2}}{1-\left(H r_{*} / c\right)^{2}}}_{h} .
\end{aligned}
$$

Hence the differentials of radar-distance and time-lapse for Einstein-simultaneity are given by

$$
\begin{aligned}
d l_{*} & =\frac{d r_{*}}{\sqrt{1-\left(H r_{*} / c\right)^{2}}}, \\
d t_{*} & =-\frac{H r_{*} / c^{2}}{1-\left(H r_{*} / c\right)^{2}} d r_{*} .
\end{aligned}
$$

Let the distinguished observer (us on earth) now move along the geodesic $r_{*}=0$. Integration of (115) from $r_{*}=$ 0 to some value $r_{*}$ then gives the radar distance $l_{*}$ as well 
as the time lapse $\Delta t_{*}$ as functions of the cosmologically simultaneous geodesic distance $r_{*}$ :

$$
\begin{aligned}
l_{*} & =(c / H) \arcsin \left(H r_{*} / c\right) \\
& =r_{*}\left\{1+\frac{1}{6}\left(H r_{*} / c\right)^{2}+\mathcal{O}\left(\left(H r_{*} / c\right)^{3}\right)\right\} \\
\Delta t_{*} & =(1 / 2 H) \ln \left(1-\left(H r_{*} / c\right)^{2}\right) \\
& =\left(r_{*} / c\right)\left\{-\frac{1}{2}\left(H r_{*} / c\right)+\mathcal{O}\left(\left(H r_{*} / c\right)^{2}\right)\right\}
\end{aligned}
$$

Combining both equations in (116) allows to express the time-lapse in terms of the radar-distance:

$$
\begin{aligned}
\Delta t_{*} & =H^{-1} \ln \left(\cos \left(H l_{*} / c\right)\right) \\
& =\left(l_{*} / c\right)\left\{-\frac{1}{2}\left(H l_{*} / c\right)+\mathcal{O}\left(\left(H l_{*} / c\right)^{2}\right)\right\} .
\end{aligned}
$$

Now, suppose a satellite $S$ moves on a worldline $r_{*}\left(t_{*}\right)$ in the neighborhood of our worldline $r_{*}=0$. Assume that we measure the distance to the satellite by radar coordinates. Then instead of the value $r_{*}$ we would use $l_{*}$ and instead of the argument $t_{*}$ we would assign the time $t_{*}-\Delta t_{*}$ which corresponds to the value of cosmological time at that event on our worldline that is Einstein synchronous to the event $\left(t_{*}, r_{*}\right)$; see Fig. 2. Hence we have

$$
\begin{aligned}
l_{*}\left(t_{*}\right) & =(c / H) \sin ^{-1}\left\{r_{*}\left(t_{*}+\Delta t_{*}\right) H / c\right\} \\
& \approx r_{*}-\frac{1}{2}(v / c)(H c)\left(r_{*} / c\right)^{2},
\end{aligned}
$$

where (118b) is (118a) to leading order and all quantities are evaluated at $t_{*}$. We set $v=\dot{r}_{*}$.

To see what this entails we Taylor expand in $t_{*}$ around $t_{*}=0$ (just a convenient choice):

$$
r_{*}\left(t_{*}\right)=r_{0}+v_{0} t_{*}+\frac{1}{2} a_{0} t_{*}^{2}+\cdots
$$

and insert in (118b). This leads to

$$
l_{*}\left(t_{*}\right)=\tilde{r}_{0}+\tilde{v}_{0} t_{*}+\frac{1}{2} \tilde{a}_{0} t_{*}^{2}+\cdots,
$$

where,

$$
\begin{aligned}
& \tilde{r}_{0}=r_{0}-(H c) \frac{1}{2}\left(v_{0} / c\right)\left(r_{0} / c\right)^{2} \\
& \tilde{v}_{0}=v_{0}-(H c)\left(v_{0} / c\right)^{2}\left(r_{0} / c\right) \\
& \tilde{a}_{0}=a_{0}-(H c)\left\{\left(v_{0} / c\right)^{3}+\left(r_{0} / c\right)\left(v_{0} / c\right)\left(a_{0} / c\right)\right\} .
\end{aligned}
$$

These are, in quadratic approximation, the sought-after relations between the quantities measured via radar tracking (tilded) and the quantities which arise in the (improved) Newtonian equations of motion (not tilded).

In particular, the last equation (121c) shows that there is an apparent inward pointing acceleration, given by $\mathrm{Hc}$ times the $(v / c)^{3}+\cdots$ term in curly brackets. As discussed in the introduction, $H c$ is indeed of the same order of magnitude as the PA, as was much emphasized in (Rosales and Sanchez-Gomez, 1998; Rosales, 2002). However, in contrast to these authors, we also get the additional term in curly brackets, which in case of the Pioneer spacecraft suppresses the $H c$ term by 13 orders

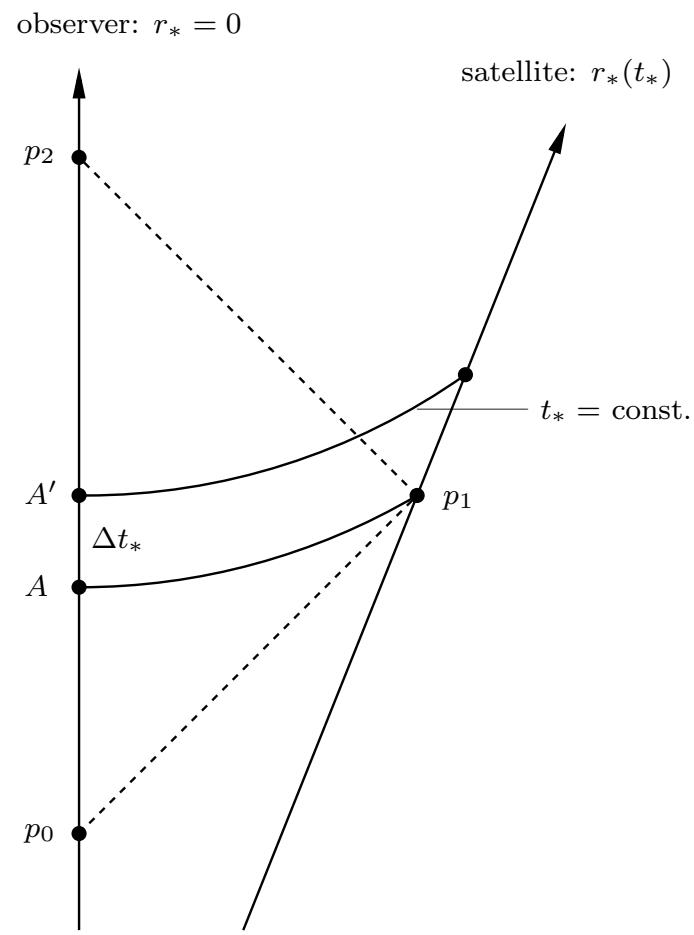

FIG. 2 An observer moves on the geodesic worldline $r_{*}=0$ and observes a satellite by exchanging electromagnetic signals (dashed line). Event $p_{0}$ corresponds to the signal emission by the observer, $p_{1}$ to its reflection by the satellite, and $p_{2}$ to its re-absorption by the observer. $t_{*}$ denotes the cosmological time and the two curved lines correspond to hypersurfaces of constant $t_{*}$. On the observer's worldline event $A$ is defined to be simultaneous to $p_{1}$ with respect to $t_{*}$ and $A^{\prime}$ is defined to lie half-way in proper time between $p_{0}$ and $p_{2}$ on the observer's worldline. In cosmological time $A^{\prime}$ is $\Delta t_{*}$ ahead of $A$. In cosmological coordinates, the satellite's trajectory is represented by the a function $t_{*} \mapsto r_{*}\left(t_{*}\right)$, where $r_{*}\left(t_{*}\right)$ denotes the proper geodesic distance in the hypersurface $t_{*}=$ const. between its intersection points with both worldlines. However, using radar coordinates, the observer takes $A^{\prime}$ to be simultaneous with $p_{1}$ and uses $l_{*}$ as measure for the satellite's simultaneous distance. Since $r_{*}$ and $l_{*}$ are related by (116a), it follows that the observer uses the function $t_{*} \mapsto l_{*}\left(r_{*}\left(t_{*}-\Delta t_{*}\right)\right)$ to characterize the satellite's trajectory, which leads to (118).

of magnitude! ${ }^{17}$ Hence we conclude that, with respect to the PA, there is no significant kinematical effect resulting from the distinct simultaneity structures inherent in radar and cosmological coordinates.

17 Our Eq. (117) corresponds to Eq. (10) of (Rosales and Sanchez-Gomez, 1998). From it the authors of (Rosales and Sanchez-Gomez, 1998) and (Rosales, 2002) immediately jump to the conclusion that there is "an effective residual acceleration directed toward the center of coordinates; its constant value is $H c$ ". We were unable to follow this conclusion. Likewise, we are unable to follow the conclusion in (Fahr and Siewert, 2008). 


\section{B. Doppler tracking in cosmological spacetimes}

Doppler Tracking is a common method of tracking the position of vehicles in space. It involves measuring the Doppler shift of an electromagnetic signal sent from a spacecraft to a tracking station on Earth. This signal is either coming from an on-board oscillator or is coherently transponded by the vehicle in response to a signal received from the ground station. Here we focus on the second of these modes, which is more useful for navigation, partly because the returning signal is measured against the same frequency reference as that of the originally transmitted signal and partly because the Earthbased frequency reference is also more stable than the oscillator on-board the spacecraft.

\section{Minkowski spacetime}

It is clear that this method will be fundamentally influenced if performed within a time varying background geometry. Before elaborating on this, we consider the simple case of static Minkowski space.

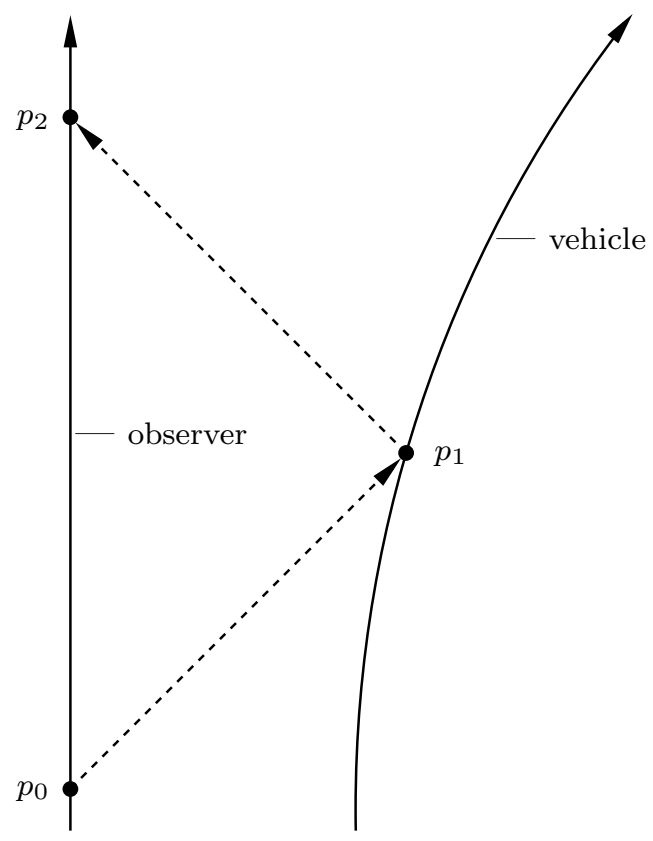

FIG. 3 Exchange of electromagnetic signals (represented by their rays at a slope of 45 degrees) between us and the space vehicle. Time runs vertically.

In Fig. 3 we depicted two worldlines, one of the observer (straight vertically) and one for the space vehicle. A light signal is emitted by the observer at the event $p_{0}$, reflected by the vehicle at event $p_{1}$, and finally received back by the observer at event $p_{2}$. We choose a global Minkowski frame, that is global coordinates $\left\{x^{\mu}\right\}=$ $\left\{t, x^{i}\right\}$ with $\boldsymbol{g}\left(\boldsymbol{\partial} / \boldsymbol{\partial} x^{\mu}, \boldsymbol{\partial} / \boldsymbol{\partial} x^{\nu}\right)=\operatorname{diag}(1,-1,-1,-1)$, in which the observer (for simplicity assumed to be inertial) is at rest at the origin of the spatial coordinates. If $\beta:=v / c$ denotes the radial velocity of the vehicle in units of $c$, the well known special-relativistic Doppler formula (applied twice) says that the ratio between the received and the emitted frequencies is ${ }^{18}$

$$
\frac{\omega_{2}\left(t_{2}\right)}{\omega_{0}\left(t_{0}\right)}=\frac{1-\beta\left(t_{1}\right)}{1+\beta\left(t_{1}\right)} .
$$

Here $t_{0}, t_{1}$, and $t_{2}$ refer to the global Minkowski time attributed to $p_{0}, p_{1}$, and $p_{2}$, respectively. In Doppler tracking one is interested in the derivative of this ratio with respect to $t_{2}$, which yields a measure for the velocity of the spacecraft. We assume $\omega_{0}$ to be constant in time and note that, given the worldlines of the observer and the vehicle, $t_{1}$ and $t_{0}$ are uniquely determined by $t_{2}$ (since the events $p_{1}$ and $p_{0}$ are determined by $p_{2}$ ). If $r$ denotes the spatial radius coordinate we have $t_{2}-t_{1}=r\left(t_{1}\right) / c$. Differentiation with respect to $t_{1}$ leads to

$$
\frac{d t_{1}}{d t_{2}}=\frac{1}{1+\beta\left(t_{1}\right)}
$$

and hence

$$
\begin{aligned}
-\frac{1}{2} \frac{\dot{\omega}_{2}\left(t_{2}\right)}{\omega_{0}\left(t_{0}\right)} & =\dot{\beta}\left(t_{1}\right)\left(1+\beta\left(t_{1}\right)\right)^{-3} \\
& =\dot{\beta}\left(t_{1}\right)\left(1-3 \beta\left(t_{1}\right)+\mathcal{O}\left(\beta^{2}\right)\right) .
\end{aligned}
$$

This shows that $-\dot{\omega}_{2} / 2 \omega_{0}$, namely (minus one-half) the derivative of the received to emitted frequency ratio with respect to the proper time of the receiving observer, gives the spacecraft's spatial acceleration up to corrections of $\operatorname{order} \beta$. Note that in view of note 18 it would be inappropriate to call these corrections 'special relativistic'.

The final goal of this section is to derive the generalization of (124) for a cosmological spacetime. For this we need two things: First, we need to know what is the generalization of the concepts of spatial velocity and spatial acceleration in an arbitrary spacetime and, second, we need to know how electromagnetic signals propagate in an arbitrary spacetime. This is taken care of in the next paragraph.

\section{General setting}

In order to generalize the notions of spatial velocity and spatial acceleration to arbitrary spacetime one needs

18 Note that in Special Relativity the Doppler Formula does, of course, not distinguish between moving emitter and moving receiver. So [122 is obtained by squaring the frequency shift $\sqrt{(1-\beta) /(1+\beta)}$, which is picked up once for the ratio $\omega_{R}\left(t_{R}\right) / \omega_{1}\left(t_{1}\right)$ (receiver moving relative to the Minkowski frame) and once for $\omega_{2}\left(t_{2}\right) / \omega_{1}\left(t_{1}\right)$ (emitter moving relative to Minkowski frame). Incidentally, exactly the same formula would result in non-relativistic physics if the observer is taken to be at rest with respect to the wave-guiding medium (e.g., the ether), which distinguishes the two states of relative motion. Indeed, in this case we have $\omega_{1}\left(t_{1}\right) / \omega_{0}\left(t_{0}\right)=\left(1-\beta\left(t_{1}\right)\right)$ and $\omega_{2}\left(t_{2}\right) / \omega_{1}\left(t_{1}\right)=1 /\left(1-\beta\left(t_{1}\right)\right)$, whose product is again just 122). 
to introduce a fiducial reference 'observer-field'; compare, e.g., (Bini et al., 1995) and also (Carrera, 2009)). An observer at the event $p$ is a future pointing unit timelike vector in the tangent space $T_{p}(\mathcal{M})$ of $\mathcal{M}$ at $p$. An $o b$ server field is a field of future pointing unit timelike vectors. Any observer $\boldsymbol{u}$ at $p$ gives rise to an orthogonal split of the tangent space $T_{p}(\mathcal{M})$ at $p$ in a part parallel to $\boldsymbol{u}$ (the local time-axis) and a part orthogonal to it (the local rest space). Since $\boldsymbol{u}$ is not lightlike the two orthogonal subspaces are complementary, that is, together they span the whole tangent space and intersect only in the zero vector. The orthogonal projections of an arbitrary vector $\boldsymbol{X} \in T_{p}(\mathcal{M})$ onto these subspaces are, respectively, given by

$$
\begin{aligned}
& Q_{u}(X):=\boldsymbol{g}(\boldsymbol{X}, \boldsymbol{u}) \boldsymbol{u} \\
& \boldsymbol{P}_{\boldsymbol{u}}(\boldsymbol{X}):=\boldsymbol{X}-\boldsymbol{g}(\boldsymbol{X}, \boldsymbol{u}) \boldsymbol{u}
\end{aligned}
$$

which imply the decomposition identity $\boldsymbol{X}=\boldsymbol{Q}_{\boldsymbol{u}}(\boldsymbol{X})+$ $\boldsymbol{P}_{\boldsymbol{u}}(\boldsymbol{X})$.

If two observers $\boldsymbol{u}$ and $\boldsymbol{v}$ are defined at the same point, the spatial velocity (over $c$ ) of $\boldsymbol{v}$ with respect to $\boldsymbol{u}$ is given by

$$
\boldsymbol{\beta}_{\boldsymbol{u}}(\boldsymbol{v}):=\frac{\boldsymbol{P}_{\boldsymbol{u}}(\boldsymbol{v})}{\left\|\boldsymbol{Q}_{\boldsymbol{u}}(\boldsymbol{v})\right\|}=\frac{\boldsymbol{v}-\boldsymbol{g}(\boldsymbol{v}, \boldsymbol{u}) \boldsymbol{u}}{\boldsymbol{g}(\boldsymbol{v}, \boldsymbol{u})}
$$

which is an element of the local rest space $\boldsymbol{P}_{\boldsymbol{u}} T(\mathcal{M})$. Its modulus is given by

$$
\beta_{\boldsymbol{u}}(\boldsymbol{v}):=\left\|\boldsymbol{\beta}_{\boldsymbol{u}}(\boldsymbol{v})\right\|=\sqrt{1-1 / \boldsymbol{g}(\boldsymbol{u}, \boldsymbol{v})^{2}} .
$$

Note that for the modulus we have $\beta_{\boldsymbol{u}}(\boldsymbol{v})=\beta_{\boldsymbol{v}}(\boldsymbol{u})$, though the vectors $\boldsymbol{\beta}_{\boldsymbol{u}}(\boldsymbol{v})$ and $\boldsymbol{\beta}_{\boldsymbol{v}}(\boldsymbol{u})$ are linearly independent as they lie in $\boldsymbol{P}_{u} T(\mathcal{M})$ and $\boldsymbol{P}_{v} T(\mathcal{M})$, respectively. Note also that $\boldsymbol{g}(\boldsymbol{u}, \boldsymbol{v})=1 / \sqrt{1-\beta_{\boldsymbol{u}}^{2}(\boldsymbol{v})}$ is just the ordinary 'gamma-factor'. Finally, if $\boldsymbol{e} \in \boldsymbol{P}_{\boldsymbol{u}} T(\mathcal{M})$ is a unit vector, we define the spatial velocity of $\boldsymbol{v}$ in direction $\boldsymbol{e}$ w.r.t. $\boldsymbol{u}$ by

$$
\boldsymbol{\beta}_{\boldsymbol{u}}^{e}(\boldsymbol{v})=-\boldsymbol{g}\left(\boldsymbol{e}, \boldsymbol{\beta}_{\boldsymbol{u}}(\boldsymbol{v})\right)=-\frac{\boldsymbol{g}(\boldsymbol{e}, \boldsymbol{v})}{\boldsymbol{g}(\boldsymbol{u}, \boldsymbol{v})} .
$$

The spatial acceleration of a worldline $\gamma$ w.r.t. a given observer field $\boldsymbol{u}$ is defined as the rate of change of the spatial velocity $\boldsymbol{\beta}_{\boldsymbol{u}}(\dot{\gamma})$ within the local rest spaces $\boldsymbol{P}_{\boldsymbol{u}} T(\mathcal{M})$ of $\boldsymbol{u}$ and with respect to the clocks moving along $\boldsymbol{u}$. Denoting this acceleration (divided by $c$ ) with $\boldsymbol{\alpha}$, we have

$$
\boldsymbol{\alpha}_{\boldsymbol{u}}(\gamma):=\nabla_{\dot{\gamma}}^{\boldsymbol{u}} \boldsymbol{\beta}_{\boldsymbol{u}}(\dot{\gamma}),
$$

where we used the following covariant derivative for $\boldsymbol{P}_{\boldsymbol{u}} T(\mathcal{M})$-valued vector fields along $\gamma$ :

$$
\nabla_{\dot{\gamma}}^{\boldsymbol{u}}:=\left\|\boldsymbol{Q}_{\boldsymbol{u}} \dot{\gamma}\right\|^{-1} \boldsymbol{P}_{\boldsymbol{u}} \circ \nabla_{\dot{\gamma}} \circ \boldsymbol{P}_{\boldsymbol{u}}
$$

Here $\nabla_{\dot{\gamma}}$ denotes the ordinary (Levi-Civita) covariant derivative along $\gamma$. As an application one can, for example, rewrite the geodesic equation, $\nabla_{\dot{\gamma}} \dot{\gamma}=0$, for a worldline $\gamma$ in terms of the spatial quantities just introduced. One gets (see (Carrera, 2009) or, in a slightly different notation, (Bini et al., 1995)):

$$
\boldsymbol{\alpha}_{u}=-\boldsymbol{S}_{\boldsymbol{\beta}_{u}}\left[\boldsymbol{a}_{\boldsymbol{u}}+\boldsymbol{\theta}_{\boldsymbol{u}}\left(\boldsymbol{\beta}_{\boldsymbol{u}}\right)+\boldsymbol{\omega}_{\boldsymbol{u}}\left(\boldsymbol{\beta}_{\boldsymbol{u}}\right)\right],
$$

where for better readability we omitted the arguments $\gamma$ and $\dot{\gamma}$ in the spatial acceleration and spatial velocity. Here $\boldsymbol{a}_{\boldsymbol{u}}:=\boldsymbol{\nabla}_{\boldsymbol{u}} \boldsymbol{u}$ is the four-acceleration of the observer field $\boldsymbol{u}, \boldsymbol{\theta}_{\boldsymbol{u}}$ and $\boldsymbol{\omega}_{\boldsymbol{u}}$ are, respectively, its shear-expansion and rotation tensors of rank $(1,1)$ (endomorphism), and $\boldsymbol{S}_{\boldsymbol{\beta}_{\boldsymbol{u}}}:=\boldsymbol{P}_{\boldsymbol{u}}+\boldsymbol{\beta}_{\boldsymbol{u}} \otimes \boldsymbol{\beta}_{\boldsymbol{u}}$ is a rank $(1,1)$ tensor which, in a slow-motion approximation (neglecting quadratic and higher terms in $\beta$ ), reduces to the identity on the local rest space of $\boldsymbol{u}$. Equation (131) should be seen as a local version of Newton's equation. For example, in Schwarzschild-deSitter spacetime (59), taking $\boldsymbol{u}$ to be proportional to the timelike Killing field $\boldsymbol{\partial} / \boldsymbol{\partial T}$, one has $\boldsymbol{\theta}_{\boldsymbol{u}}=\boldsymbol{\omega}_{\boldsymbol{u}}=0$ (because of the Killing equation and spherical symmetry, respectively) and

$$
\boldsymbol{a}_{\boldsymbol{u}}=\nabla_{\boldsymbol{u}} \boldsymbol{u}=\frac{1}{\sqrt{V}}\left(\frac{m}{R^{2}}-\frac{\Lambda}{3} R\right) \boldsymbol{e}_{R},
$$

where $\boldsymbol{e}_{R}$ denotes the the normalized radial vector field $\boldsymbol{\partial} / \boldsymbol{\partial} R$ (we use here the coordinates and the notation of (59) ). Hence, in slow-motion and weak-field approximation (that is keeping only linear terms in $\beta, m / R$, and $\Lambda R^{2}$ ), the geodesic equation of motion in the form (131) reduces to

$$
\boldsymbol{\alpha}_{\boldsymbol{u}} \approx\left(-\frac{m}{R^{2}}+\frac{\Lambda}{3} R\right) \boldsymbol{e}_{R},
$$

which just gives the 'improved' Newtonian equation for geodesic motions in Schwarzschild-deSitter spacetime. It has the same form as the improved Newtonian equation studied in Section III.

We turn now to electromagnetic signals and restrict our attention to monochromatic waves in the geometricoptics approximation (i.e. for wave-lengths negligibly small w.r.t. a typical radius of curvature of the spacetime and w.r.t. a typical length over which amplitude, polarization, and frequency vary). In this approximation an electromagnetic signal propagates on a lightlike geodesic along which the wave-vector, $\boldsymbol{k}$, is tangent, future-pointing, and parallelly transported. Recall that $\boldsymbol{k}$ is so normalized that the frequency measured by an observer, say $\boldsymbol{u}$, is

$$
\omega_{\boldsymbol{u}}(\boldsymbol{k}):=\boldsymbol{g}(\boldsymbol{u}, \boldsymbol{k}) .
$$

Given a wave-vector $\boldsymbol{k}$ and two observers $\boldsymbol{u}, \boldsymbol{v}$ at the same spacetime point, their observed frequencies are thus $\omega_{\boldsymbol{v}}(\boldsymbol{k})=\boldsymbol{g}(\boldsymbol{v}, \boldsymbol{k})$ and $\omega_{\boldsymbol{u}}(\boldsymbol{k})=\boldsymbol{g}(\boldsymbol{u}, \boldsymbol{k})$, and their ratio is given by

$$
\frac{\omega_{\boldsymbol{v}}(\boldsymbol{k})}{\omega_{\boldsymbol{u}}(\boldsymbol{k})}=\frac{\boldsymbol{g}\left(\boldsymbol{Q}_{\boldsymbol{u}} \boldsymbol{v}+\boldsymbol{P}_{\boldsymbol{u}} \boldsymbol{v}, \boldsymbol{k}\right)}{\boldsymbol{g}(\boldsymbol{u}, \boldsymbol{k})}=g(\boldsymbol{u}, \boldsymbol{v})\left[1-\beta_{\boldsymbol{u}}^{\hat{\boldsymbol{k}}}(\boldsymbol{v})\right] .
$$


Here the spacelike unit vector $\hat{\boldsymbol{k}}:=\left\|\boldsymbol{P}_{\boldsymbol{u}}(\boldsymbol{k})\right\|^{-1} \boldsymbol{P}_{\boldsymbol{u}}(\boldsymbol{k})$ defines the direction of $\boldsymbol{k}$ in the local rest space of $\boldsymbol{u}$. In deriving (135) we used (128) and $\left\|\boldsymbol{P}_{\boldsymbol{u}}(\boldsymbol{k})\right\|=\boldsymbol{g}(\boldsymbol{u}, \boldsymbol{k})$ to write $\boldsymbol{g}\left(\boldsymbol{v}, \boldsymbol{P}_{\boldsymbol{u}}(\boldsymbol{k})\right)=-\boldsymbol{g}(\boldsymbol{v}, \boldsymbol{u}) \boldsymbol{g}(\boldsymbol{u}, \boldsymbol{k}) \beta_{\boldsymbol{u}}^{\hat{\boldsymbol{k}}}(\boldsymbol{v})$. Equation (135) is the general form of the Doppler formula.

Let now $\boldsymbol{u}$ be an observer field along one integral line of which the distinguished observer is moving. The worldline of the vehicle is denoted by $\gamma$. The domain of the field $\boldsymbol{u}$ is assumed to include a neighborhood of $\gamma$. The wave-vector $\boldsymbol{k}_{0}$ emitted at $p_{0}$ suffers three changes:

1. propagation from $p_{0}$ to $p_{1}: \boldsymbol{k}_{0} \rightarrow \boldsymbol{k}_{1}$;

2. reflection at $p_{1}: \boldsymbol{k}_{1} \rightarrow \boldsymbol{k}_{1}^{\prime}$;

3. propagation from $p_{1}$ to $p_{2}: \boldsymbol{k}_{1}^{\prime} \rightarrow \boldsymbol{k}_{2}$.

We are interested in the ratio of the received to the emitted frequency:

$$
\frac{\omega_{2}}{\omega_{0}}=\frac{\boldsymbol{g}\left(\boldsymbol{u}_{2}, \boldsymbol{k}_{2}\right)}{\boldsymbol{g}\left(\boldsymbol{u}_{0}, \boldsymbol{k}_{0}\right)}=\left(\frac{\omega_{2}}{\omega_{1}^{\prime}}\right)\left(\frac{\omega_{1}^{\prime}}{\omega_{1}}\right)\left(\frac{\omega_{1}}{\omega_{0}}\right) \text {. }
$$

What happens at reflection (the second process: $\boldsymbol{k}_{1} \rightarrow$ $\left.\boldsymbol{k}_{1}^{\prime}\right)$ ? Well, with respect to the spacecraft moving along $\gamma$ with four-velocity $\boldsymbol{v}=\dot{\boldsymbol{\gamma}}$, the wave vector $\boldsymbol{k}_{1}$ at $p_{1}$ splits according to

$$
\boldsymbol{k}_{1}=\boldsymbol{Q}_{\dot{\gamma}}\left(\boldsymbol{k}_{1}\right)+\boldsymbol{P}_{\dot{\gamma}}\left(\boldsymbol{k}_{1}\right) .
$$

A corner-cube reflector transported along $\gamma$ will reverse $\boldsymbol{P}_{\dot{\boldsymbol{\gamma}}}\left(\boldsymbol{k}_{1}\right)$ while keeping $\boldsymbol{Q}_{\dot{\boldsymbol{\gamma}}}\left(\boldsymbol{k}_{1}\right)$ intact (here we neglect a possible transponder shift which is irrelevant for our discussion):

$$
\boldsymbol{k}_{1} \mapsto \boldsymbol{k}_{1}^{\prime}=\boldsymbol{Q}_{\dot{\gamma}}\left(\boldsymbol{k}_{1}\right)-\boldsymbol{P}_{\dot{\gamma}}\left(\boldsymbol{k}_{1}\right)=2 \boldsymbol{Q}_{\dot{\gamma}}\left(\boldsymbol{k}_{1}\right)-\boldsymbol{k}_{1} .
$$

Hence $\omega_{1}:=\omega_{\boldsymbol{u}}\left(\boldsymbol{k}_{1}\right)=\boldsymbol{g}\left(\boldsymbol{u}_{1}, \boldsymbol{k}_{1}\right)$ and $\omega_{1}^{\prime}:=\omega_{\boldsymbol{u}}\left(\boldsymbol{k}_{1}^{\prime}\right)=$ $\boldsymbol{g}\left(\boldsymbol{u}_{1}, \boldsymbol{k}_{1}^{\prime}\right)$, the in- and out-going frequencies measured by the observer $\boldsymbol{u}$ at $p_{1}$, are related by

$$
\frac{\omega_{1}^{\prime}}{\omega_{1}}=2 \frac{\left.\boldsymbol{g}(\boldsymbol{u}, \dot{\gamma}) \boldsymbol{g}(\dot{\gamma}, \boldsymbol{k})\right|_{p_{1}}}{\left.\boldsymbol{g}(\boldsymbol{u}, \boldsymbol{k})\right|_{p_{1}}}-1=2 \frac{1-\left.\boldsymbol{\beta}_{\boldsymbol{u}}^{\hat{\boldsymbol{k}}}(\dot{\gamma})\right|_{p_{1}}}{1-\left.\beta_{u}^{2}(\dot{\gamma})\right|_{p_{1}}}-1
$$

where in the last step we just used (135) to rewrite the ratio $\boldsymbol{g}(\dot{\gamma}, \boldsymbol{k}) / \boldsymbol{g}(\boldsymbol{u}, \boldsymbol{k})$. This accounts for the middle ratio on the right-hand side of (136).

To account for the other two ratios in (136), one uses the laws of geometric optics in (curved) spacetime to relate $\omega_{0}=\boldsymbol{g}\left(\boldsymbol{u}_{0}, \boldsymbol{k}_{0}\right)$ (at $\left.p_{0}\right)$ and $\omega_{2}=\boldsymbol{g}\left(\boldsymbol{u}_{2}, \boldsymbol{k}_{2}\right)$ (at $p_{2}$ ) to kinematical quantities of $\gamma$ at $p_{1} .{ }^{19}$ For example, if $\boldsymbol{u}$ is a Killing field (like $\boldsymbol{u}=\boldsymbol{\partial} / \boldsymbol{\partial t}$ in Special Relativity), we have $\boldsymbol{g}\left(\boldsymbol{u}_{0}, \boldsymbol{k}_{0}\right)=\boldsymbol{g}\left(\boldsymbol{u}_{1}, \boldsymbol{k}_{1}\right)$ and $\boldsymbol{g}\left(\boldsymbol{u}_{2}, \boldsymbol{k}_{2}\right)=\boldsymbol{g}\left(\boldsymbol{u}_{1}, \boldsymbol{k}_{1}^{\prime}\right)$, so that

$$
\frac{\omega_{2}}{\omega_{0}}=2 \frac{1-\left.\beta_{\boldsymbol{u}}^{\hat{\boldsymbol{k}}}(\dot{\gamma})\right|_{p_{1}}}{1-\left.\beta_{\boldsymbol{u}}^{2}(\dot{\gamma})\right|_{p_{1}}}-1
$$

As a trivial application, this includes the generalized form of (122), the latter corresponding to purely radial motion.

19 In general spacetimes without timelike conformal Killing fields these quotients will also explicitly depend on time.

\section{FLRW spacetimes}

In standard cosmological spacetimes (FLRW), $\boldsymbol{u}=$ $\boldsymbol{\partial} / \boldsymbol{\partial} t$ is not Killing, though $\boldsymbol{X}=a(t) \boldsymbol{\partial} / \boldsymbol{\partial} t$ is conformally Killing $\left(L_{\boldsymbol{X}} \boldsymbol{g}=2 \dot{a} \boldsymbol{g}\right)$. One now has $a_{0} \boldsymbol{g}\left(\boldsymbol{u}_{0}, \boldsymbol{k}_{0}\right)=$ $a_{1} \boldsymbol{g}\left(\boldsymbol{u}_{1}, \boldsymbol{k}_{1}\right)$ and $a_{2} \boldsymbol{g}\left(\boldsymbol{u}_{2}, \boldsymbol{k}_{2}\right)=a_{1} \boldsymbol{g}\left(\boldsymbol{u}_{1}, \boldsymbol{k}_{1}^{\prime}\right)$, so that instead of (140) one gets

$$
\frac{\omega_{2}}{\omega_{0}}=\frac{a_{0}}{a_{2}}\left\{2 \frac{1-\left.\beta_{u}^{\hat{k}}(\dot{\gamma})\right|_{p_{1}}}{1-\left.\beta_{u}^{2}(\dot{\gamma})\right|_{p_{1}}}-1\right\} .
$$

We now want to relate the $t_{2}$-derivative of (141) to the acceleration of $\gamma$. In order to calculate the derivative $\dot{\omega}_{2}\left(t_{2}\right) / \omega_{0}\left(t_{0}\right)$ we need to know the derivatives $d t_{1} / d t_{2}$ and $d t_{0} / d t_{2}$. Restricting to the flat FLRW case for simplicity, they follow from the law of null propagation:

$$
\begin{aligned}
& \int_{t_{1}\left(t_{2}\right)}^{t_{2}} \frac{d t}{a(t)}=-\frac{1}{c} \int_{r_{1}\left(t_{1}\left(t_{2}\right)\right)}^{r_{2}} d r \\
& \int_{t_{0}\left(t_{2}\right)}^{t_{2}} \frac{d t}{a(t)}=\frac{1}{c}\left\{\int_{r_{0}}^{r_{1}\left(t_{1}\left(t_{2}\right)\right)} d r-\int_{r_{1}\left(t_{1}\left(t_{2}\right)\right)}^{r_{2}} d r\right\} .
\end{aligned}
$$

Differentiation with respect to $t_{2}$ yields, respectively

$$
\begin{aligned}
& \frac{d t_{1}}{d t_{2}}=\frac{a\left(t_{1}\right)}{a\left(t_{2}\right)}\left(1+\left.\beta_{\boldsymbol{u}}^{\hat{\boldsymbol{k}}}(\dot{\gamma})\right|_{p_{1}}\right)^{-1}, \\
& \frac{d t_{0}}{d t_{2}}=\frac{a\left(t_{0}\right)}{a\left(t_{2}\right)} \frac{1-\left.\beta_{\boldsymbol{u}}^{\hat{\boldsymbol{k}}}(\dot{\gamma})\right|_{p_{1}}}{1+\left.\beta_{\boldsymbol{u}}^{\hat{\boldsymbol{k}}}(\dot{\gamma})\right|_{p_{1}}} .
\end{aligned}
$$

The exact formula for the $t_{2}$-derivative of the frequency-shift rate can now be computed. One obtains

$$
\begin{aligned}
& -\frac{\dot{\omega}_{2}\left(t_{2}\right)}{\omega_{0}\left(t_{0}\right)}= \\
& \frac{a_{0}}{a_{2}}\left\{2\left[\alpha^{\hat{\boldsymbol{k}}}-\boldsymbol{g}\left(\boldsymbol{\beta}, \nabla_{\dot{\boldsymbol{\gamma}}}^{\boldsymbol{u}} \hat{\boldsymbol{k}}\right)\right] \frac{a_{1}}{a_{2}}\left[1+\beta^{\hat{k}}\right]^{-1}\left[1-\beta^{2}\right]^{-1}\right. \\
& +4 \boldsymbol{g}(\boldsymbol{\alpha}, \boldsymbol{\beta}) \frac{a_{1}}{a_{2}}\left[\frac{1-\beta^{\hat{k}}}{1+\beta^{\hat{k}}}\right]\left[1-\beta^{2}\right]^{-2} \\
& \left.+\left[\frac{\dot{a}_{2}}{a_{2}}-\frac{\dot{a}_{0}}{a_{2}}\left(\frac{1-\beta^{\hat{k}}}{1+\beta^{\hat{k}}}\right)\right]\left[\frac{1-2 \beta^{\hat{k}}+\beta^{2}}{1-\beta^{2}}\right]\right\},
\end{aligned}
$$

where we suppressed the argument $\dot{\gamma}$ and index $\boldsymbol{u}$ at $\beta$ for better readability. This formula provides an exact relation between the time derivative of the observable frequency shift (defined 'here') and the kinematical quantities of the vehicle (defined 'there'), provided the scale function $a(t)$ is known. For purely radial motion $\nabla_{\dot{\gamma}}^{u} \hat{\boldsymbol{k}}=0$ and we obtain the simpler expression (now writing $\alpha$ for $\alpha^{\hat{k}}$ )

$$
\begin{aligned}
&-\frac{1}{2} \frac{\dot{\omega}_{2}\left(t_{2}\right)}{\omega_{0}\left(t_{0}\right)}=-\frac{a_{0} a_{1}}{a_{2}^{2}}\left\{\alpha(1+\beta)^{-3}+\right. \\
&\left.\frac{1}{2}\left[\frac{\dot{a}_{2}}{a_{1}}-\frac{\dot{a}_{0}}{a_{1}}\left(\frac{1-\beta}{1+\beta}\right)\right]\left[\frac{1-\beta}{1+\beta}\right]\right\} .
\end{aligned}
$$


In order to consistently approximate this expression in terms of small quantities $\beta$ and $H \Delta t$, where $\Delta t:=$ $\left(t_{2}-t_{0}\right) / 2$, we think of (145) as being multiplied with $\Delta t$ and regard $\alpha \Delta t$ as being of order $\beta$. Then, keeping only quadratic terms in $\beta$, linear terms in $H \Delta t$ where $\Delta t:=\left(t_{2}-t_{0}\right) / 2$, and also mixed terms $\beta H \Delta t$, we get

$$
-\frac{1}{2} \frac{\dot{\omega}_{2}\left(t_{2}\right)}{\omega_{0}\left(t_{0}\right)} \approx \alpha(1-3 \beta-3 H \Delta t)+H \beta .
$$

Hence we see that in this approximation there are two modifications, besides the $-3 \beta$-term already familiar from (124), due to cosmic expansion: First, there is an additional contribution $-3 H \Delta t$ acting in the same way as the $-3 \beta$-term. It can also be interpreted in the same fashion, as its corresponds to the velocity (over $c$ ) of $H \Delta t$ that a comoving systems picks up during the time the signal went from the observer to the vehicle. Second, there is a constant contribution $H \beta$ to acceleration $/ c$, i.e. $H c \beta$ to acceleration, in a direction parallel to the radial velocity (i.e. outward pointing if the vehicle recedes from the observer). Hence it acts opposite to the PA and is smaller in modulus by a factor of $\beta$. Applied to the Pioneer spacecrafts, the $H \Delta t$-term amounts to a tiny 'anomalous' acceleration of $\Delta a / a<10^{-12}$, the $H c \beta$-term to $\Delta a / a<10^{-7}$.

A final point must be made regarding the choice of the reference observer-field on which the kinematic quantities related to the spacecraft (spatial velocity and spatial acceleration) and the electromagnetic signal (frequency and spatial propagation direction) crucially depend. In the Minkowskian case the reference field was just $\boldsymbol{u}=\boldsymbol{\partial} / \boldsymbol{\partial} t$, which is inertial, that is, geodesic and of vanishing rotation, shear, and expansion. It is clear that in a general spacetime such observer fields do not exist and there is no natural choice to replace them. However, in the case of spherical symmetry there is, in fact, a distinguished observer field, namely that one whose orbits lie within the timelike hypersurfaces of constant areal radius and there run perpendicular to the orbits of the rotation group. This clearly defines a non-rotating and 'non-expanding' (w.r.t. the areal radius) reference field. It is the normalization of the so-called Kodama vector field, which we discuss in detail in Appendix D.3. In a FLRW spacetime it is just given by (113). Notice that in the present case, where the hypersurfaces of constant cosmological time $t$ are flat, the areal radius corresponds also to the proper distance. Hence the integral curves of $\boldsymbol{u}_{*}$ intersect the hypersurface of constant cosmological time at constant spatial geodesic distance. More precisely, the expansion and the shear scalar of $\boldsymbol{u}_{*}$ are given by $\theta_{*}=R^{2} H \dot{H} /\left(1-(R H)^{2}\right)^{3 / 2}$ and $\sigma_{*}=-\theta_{*} / 3$, respectively, showing that they are of order $H^{3}$ which we neglect. In passing we remark that the expansion and shear of $\boldsymbol{u}_{*}$ exactly vanish for the de Sitter case, whose metric in 'static' coordinates is given by (59) for $m=0$. In this case $\boldsymbol{\partial} / \boldsymbol{\partial} t_{*}=\boldsymbol{\partial} / \boldsymbol{\partial} T$, that is, $\boldsymbol{u}_{*}$ is proportional to the timelike Killing vector field $\boldsymbol{\partial} / \boldsymbol{\partial} T$; see (59). Coming back to the general FLRW case, the acceleration of $\boldsymbol{u}_{*}$ is given by $\boldsymbol{a}_{\boldsymbol{u}_{*}}=\left(-R \ddot{a} / a+R^{3} H^{4}\right) /\left(1-(R H)^{2}\right)^{3 / 2} \boldsymbol{e}_{*}$, where $\boldsymbol{e}_{*}$ is the unit vector field orthogonal to $\boldsymbol{u}_{*}$ and to the two-sphere, pointing in positive radial direction. Hence, in the slow-motion and weak-field approximation of Eq. (146), but keeping also quadratic terms in $H$, the geodesic equation in the form (131) w.r.t. the observer field $\boldsymbol{u}_{*}$ reads as

$$
\boldsymbol{\alpha}_{\boldsymbol{u}_{*}}(\gamma) \approx\left(\frac{\ddot{a}}{a} r_{*}\right) \boldsymbol{e}_{*} \circ \gamma
$$

This is just an alternative derivation of the acceleration term (7). We point out that had we we taken (110) as observer field we would have arrived at the equation of motion $\boldsymbol{\alpha}_{\boldsymbol{u}}(\gamma) \approx-H \boldsymbol{\beta}_{\boldsymbol{u}}(\gamma)$ instead of (147), that is, no acceleration term (7) would have resulted.

In the approximation within which (146) is derived this equation remains valid if the quantities in it are reinterpreted so as to refer to $\boldsymbol{u}_{*}$ instead of $\boldsymbol{u}$. Hence we may sum up the situation by saying that equations (146) and (147) give, respectively, the two-way Doppler-tracking formula and the 'Newtonian' equation in a FLRW spacetime within the mentioned approximation.

\section{McVittie spacetime}

The same analysis can be generalized from the spatially flat FLRW spacetime (30) to the spatially flat McVittie spacetime (68). Here the observer moves along $\boldsymbol{\partial} / \boldsymbol{\partial} t$, which is not geodesic. The coordinate $t$ does now not measure proper time, denoted by $\tau$, along the observer's worldline. The result corresponding to (146) can now be stated as follows:

$$
-\frac{1}{2} \frac{\dot{\omega}_{2}\left(\tau_{2}\right)}{\omega_{0}\left(\tau_{0}\right)} \approx \alpha\left(1-3 \beta-3 \Delta \tau\left(H-m_{0} c / R^{2}\right)\right)+H \beta .
$$

Here $R$ denotes the areal radius of the observer during the measurement. Note that even though it changes along the observer's worldline according to (90), we do not need to account for the corresponding change in $\Delta \tau m_{0} c / R^{2}$ of $\left(-2 \Delta \tau m_{0} c / R^{2}\right)(H \Delta \tau)$ which is of subleading order. The additional term in (148) has a straightforward interpretation in terms of the acceleration that the observer necessarily experiences while keeping a constant radius $R$ away from the central inhomogeneity.

As for the FLRW case, we chose the observer field to which we refer the spatial quantities to be proportional to the Kodama vector field (along which the areal radius is constant). Putting $r_{*}(t, r):=A^{2}(t, r) a(t) r$ and $t_{*}(t, r):=t$, a short computation shows that the vector field $\boldsymbol{\partial} / \boldsymbol{\partial} t_{*}$ is again given by (112). In the slow-motion and weak-field approximation used in SectionV.D.2 the geodesic equation in the form (131) w.r.t. the observer field $\boldsymbol{u}_{*}$ reads

$$
\boldsymbol{\alpha}_{\boldsymbol{u}_{*}}(\gamma) \approx\left(\frac{\ddot{a}}{a} r_{*}-\frac{m_{0}}{r_{*}^{2}}\right) \boldsymbol{e}_{*} \circ \gamma
$$


where again $\boldsymbol{e}_{*}$ denotes the unit outward-pointing vector field orthogonal to $\boldsymbol{u}_{*}$ and to the two-spheres of symmetry. This is an alternative derivation of the improved Newtonian equation for the McVittie spacetime carried out in Section V.D.2. Notice that, again, within the approximations used, relation (148) remains valid if one refers the quantities to $\boldsymbol{u}_{*}$ instead of to $\boldsymbol{u}$. Therefore (148) and (149) give the two-way Doppler-tracking formula and the improved 'Newtonian' equation for the McVittie spacetime within the mentioned approximation.

In the special case of purely radial motion, insertion of (149) into (148) leads to a formula predicting the twoway Doppler-shift rate in linear order in $H \Delta \tau$ and $m_{0} / r_{*}$, and quadratic order in $\beta_{\boldsymbol{u}_{*}}(\dot{\gamma})$ :

$$
-\frac{1}{2} \frac{\dot{\omega}_{2}\left(\tau_{2}\right)}{\omega_{0}\left(\tau_{0}\right)}=-\frac{m_{0}}{r_{*}^{2}}\left(1-3 \beta^{\hat{\boldsymbol{k}}}\right)+H \beta^{\hat{\boldsymbol{k}}} .
$$

Hence there are two corrections to the Newtonian contribution. One is proportional to $H$ and stems from the cosmological expansion, the other, already familiar from the special-relativistic treatment (124), is independent of $H$ and merely due to the finiteness of the propagation speed of light (recall note 18). Their ratio is (up to a factor $\sqrt{3}$ ) given by the square of the ratio of $r_{*}$ to the geometric mean of the Schwarzschild radius $m_{0}$ and the Hubble radius $c / H$. The latter is of the order of $10^{23} \mathrm{~km}$, so that its geometric mean with a Schwarzschild radius of one kilometer is approximately given by 2400 astronomical units. The ratio of the effects is therefore of the order $10^{-7}$. Hence the cosmological contribution is negligible for any application in the Solar System as compared to the $3 \beta$-correction. For the Pioneer $10 \& 11$ spacecrafts we have a radial velocity of about $12 \mathrm{Km} / \mathrm{s}$. This amounts to a $3 \beta$-correction of magnitude $4 \cdot 10^{-5}$ times the Newtonian gravitational acceleration, in an outwardpointing direction. This is indeed of the same order of magnitude as the PA but directed oppositely.

\section{SUMMARY AND OUTLOOK}

We think it is fair to say that there are no theoretical hints that point towards a dynamical influence of cosmological expansion comparable in size to, say, that of the anomalous acceleration of the Pioneer spacecrafts. There seems to be no controversy over this point, though for completeness it should be mentioned that there exist speculations (Palle, 2005) according to which it might become relevant for future missions. But such speculations are often based on models which are not easily related to the intended physical situation, like that of Gautreau (Gautreau, 1984). Rather, as the $(\ddot{a} / a)$ improved Newtonian analysis in Section III together with its justification given in the subsequent Sections shows, there is no genuine relativistic effect coming from cosmological expansion at the levels of precision envisaged here.
On the other hand, as regards kinematical effects, the situation is less unanimous. It is very important to unambiguously understand what is meant by 'mapping out a trajectory', i.e. how to assign 'times' and 'distances'. Eventually we compare a functional relation between 'distance' and 'time' with observed data. That relation is obtained by solving some equations of motion and it has to be carefully checked whether the methods by which the tracking data are obtained match the interpretation of the coordinates in which the analytical problem is solved. In our way of speaking, dynamical effects really influence the worldline of the object in question whereas kinematical effects change the way in which one and the same worldline is mapped out from another worldline representing the observer. Here we have derived exact results concerning the influence of cosmic expansion on this mapping procedure, which allow to reliably estimate upper bounds on their magnitude. They turn out to be too small to be of any relevance in current satellite trackings, which is in accord with naive expectation but in contrast to some statements found in the literature.

At this point it is useful to recall once more the general philosophy behind such statements: From the Einstein-Straus solution it is clear that local overdensities inhibit cosmic expansion, or at least that part of it which is not due to a cosmological constant. Also, as already mentioned before, the effect of anisotropies is also to diminish the effect of global expansion (see, e.g., (Dominguez and Gaite, 2001)). Hence calculating such an effect in simple models like the improved Newtonian equation discussed in Section II.A (backed up by the various justifications we discussed in detail) clearly means to overestimate the impact of cosmic expansion in a realistic situation, where the single overdensity (e.g. representing the Sun) is surrounded by more overdense structures (the Solar-System environment, the Galaxy, etc.) with less symmetry. If this overestimation gives an already insignificant upper bound for the envisaged effect, we can conclude that it becomes even more insignificant in more realistic models.

Satellite navigation is clearly not the only potential source of interest in the question of how local inhomogeneities affect cosmological expansion. Many predictions concerning cosmological data rely on computations within the framework of the standard homogeneous and isotropic models, without properly estimating the possible effects of local inhomogeneities. Such an estimation would ideally be based on an exact inhomogeneous solution to Einstein's equations, or at least a fully controlled approximation to such a solution. The dynamical and kinematical impact of local inhomogeneities might essentially influence our interpretation of cosmological observations. As an example we mention recent serious efforts to interpret the same data that are usually taken to prove the existence of a positive cosmological constant $\Lambda$ in a context with realistic inhomogeneities (Buchert, 2000; Räsänen, 2006; Wiltshire, 2007), i.e. taking into account that cosmological param- 
eters are dressed (Buchert and Carfora, 2003). See also (Buchert, 2008) for a recent review. One might speculate that the measured $\Lambda$ can eventually be fully reduced to the action of inhomogeneities, as suggested in in (Wiltshire, 2007, 2008). For an earlier advance in this direction, see (Célérier, 2000).

\section{Acknowledgments}

This work was partially supported by the European Space Agency (ESA) under the Ariadna scheme of the Advanced Concepts Team, contract 18913/05/NL/MV. We are grateful to the ESA and the Albert-EinsteinInstitute in Golm for their support and hospitality. D.G. acknowledges support from the QUEST Excellence Cluster. We also thank Claus Lämmerzahl and Hartmann Römer for useful discussions and pointing out relevant references as well as the referees for also suggesting improvements and references.

\section{APPENDIX A: Notation, conventions, and generalities}

A model for spacetime consists of a tuple $(\mathcal{M}, \boldsymbol{g})$, where $M$ is a four-dimensional manifold and $\boldsymbol{g}$ a Lorentzian metric whose signature we take to be $(+,-,-,-)$, i.e. we use the 'mostly minus' convention. Throughout we denote geometric objects, like tensor fields and covariant derivative operators, by bold-faced letters or words. The unique metric preserving and torsion-free covariant derivative associated with $\boldsymbol{g}$ will be denoted by $\boldsymbol{\nabla}$ and the covariant derivative in the direction of a vector $\boldsymbol{X}$ by $\nabla_{\boldsymbol{X}}$. For a smooth tensor field $\boldsymbol{T}$ on $M$ its covariant derivative $\boldsymbol{\nabla} \boldsymbol{T}$ defines a linear map, $\boldsymbol{X} \mapsto \boldsymbol{\nabla}_{\boldsymbol{X}} \boldsymbol{T}$, from the tangent space to the tensor space at each point of $\mathcal{M}$ where $\boldsymbol{T}$ is defined. Since $\boldsymbol{\nabla} \boldsymbol{T}$ is again a tensor field (of rank $(p, q+1)$ if the rank of $\boldsymbol{T}$ was $(p, q)$ ) we can form $\boldsymbol{\nabla} \boldsymbol{\nabla} \boldsymbol{T}:=\boldsymbol{\nabla}(\boldsymbol{\nabla} \boldsymbol{T})$. Note that $(\boldsymbol{\nabla} \boldsymbol{\nabla} \boldsymbol{T})(\boldsymbol{X}, \boldsymbol{Y})=$ $\nabla_{X} \nabla_{Y} \boldsymbol{T}-\nabla_{\nabla_{X}} \boldsymbol{T}$. For a scalar function $f$ on $\mathcal{M}$ we have $\boldsymbol{\nabla} f=\boldsymbol{d} f$, the ordinary exterior differential, and $\boldsymbol{\nabla} \nabla f=\operatorname{Hess}(f)$, the Hessian of $f$. The metric $\boldsymbol{g}$ allows to uniquely associate to any vector $\boldsymbol{X}$ a linear form $\underline{\boldsymbol{X}}:=\boldsymbol{g}(\boldsymbol{X}, \cdot)$, called the dual (with respect to $\boldsymbol{g}$ ) of $\boldsymbol{X}$. The inverse of this map will be denoted by an overline. The gradient of a function $f$ is then $\operatorname{grad} f=\overline{\boldsymbol{d} f}$. The metricity of $\nabla$ implies that the latter commutes with the maps $\doteq$ and - , e.g. it holds: $\nabla_{X} \underline{\boldsymbol{Y}}=\underline{\nabla_{X} \boldsymbol{Y}}$. The scalar product induced by $\boldsymbol{g}$ on the tensor bundle will be denoted by $\langle\cdot, \cdot\rangle$.

Associated with any two linearly independent vectors $\boldsymbol{X}, \boldsymbol{Y}$ at a point $p \in \mathcal{M}$ is a curvature endomorphism, $\boldsymbol{R}(\boldsymbol{X}, \boldsymbol{Y})$, of the tangent space at $p$ :

$$
\begin{aligned}
\boldsymbol{R}(\boldsymbol{X}, \boldsymbol{Y}) \boldsymbol{Z} & =(\boldsymbol{\nabla} \nabla \boldsymbol{Z})(\boldsymbol{X}, \boldsymbol{Y})-(\boldsymbol{\nabla} \boldsymbol{\nabla} \boldsymbol{Z})(\boldsymbol{Y}, \boldsymbol{X}) \\
& =\nabla_{\boldsymbol{X}} \boldsymbol{\nabla}_{\boldsymbol{Y}} \boldsymbol{Z}-\boldsymbol{\nabla}_{\boldsymbol{Y}} \boldsymbol{\nabla}_{\boldsymbol{X}} \boldsymbol{Z}-\boldsymbol{\nabla}_{[\boldsymbol{X}, \boldsymbol{Y}]} \boldsymbol{Z}
\end{aligned}
$$

The Riemann- or curvature tensor, Riem, is then defined by

$$
\operatorname{Riem}(\boldsymbol{W}, \boldsymbol{Z}, \boldsymbol{X}, \boldsymbol{Y}):=\boldsymbol{g}(\boldsymbol{W}, \boldsymbol{R}(\boldsymbol{X}, \boldsymbol{Y}) \boldsymbol{Z}) .
$$

It is antisymmetric under the exchange $\boldsymbol{X} \leftrightarrow \boldsymbol{Y}$ or $\boldsymbol{W} \leftrightarrow$ $\boldsymbol{Z}$ and symmetric under the slotwise exchange of pairs $(\boldsymbol{W}, \boldsymbol{Z}) \leftrightarrow(\boldsymbol{X}, \boldsymbol{Y})$. Moreover, the antisymmetrization over any three slots vanishes (first Bianchi identity). The Ricci tensor, Ric, is defined by the trace of the following endomorphism

$$
\boldsymbol{R i c}(\boldsymbol{Y}, \boldsymbol{Z}):=\operatorname{tr}(\boldsymbol{X} \mapsto \boldsymbol{\operatorname { R i e m }}(\boldsymbol{X}, \boldsymbol{Y}) \boldsymbol{Z}),
$$

which is symmetric under exchange $\boldsymbol{Y} \leftrightarrow \boldsymbol{Z}$. The scalar curvature is defined by taking the trace of $\mathbf{R i c}$, also called the Ricci scalar, with respect to $\boldsymbol{g}$ (since Ric is not an endomorphism, we need the metric to define its trace)

$$
\mathrm{Scal}=\operatorname{tr}_{\boldsymbol{g}}(\mathbf{R i c}) \text {. }
$$

Finally, the Einstein tensor is the following combination of Ric and Scal:

$$
\text { Ein }:=\text { Ric }-\frac{1}{2} \text { Scal } \boldsymbol{g}
$$

Associated to any spacelike or timelike twodimensional plane $\Pi$ in the tangent space at $p \in \mathcal{M}$ is the sectional curvature. Its geometric interpretation is just that of the ordinary Gaussian curvature at $p$ of the two-dimensional surface in $\mathcal{M}$ that is spanned by the geodesic curves through $p$ tangent to $\Pi$. In terms of Riem it reads

$$
k_{I}:=\frac{\operatorname{Riem}(\boldsymbol{X}, \boldsymbol{Y}, \boldsymbol{X}, \boldsymbol{Y})}{\boldsymbol{Q}(\boldsymbol{X}, \boldsymbol{Y})},
$$

where $\boldsymbol{X}, \boldsymbol{Y}$ are any two linear independent vectors in $\Pi$ and

$$
\begin{aligned}
\boldsymbol{Q}(\boldsymbol{X}, \boldsymbol{Y}): & =\boldsymbol{g}(\boldsymbol{X}, \boldsymbol{X}) \boldsymbol{g}(\boldsymbol{Y}, \boldsymbol{Y})-\boldsymbol{g}(\boldsymbol{X}, \boldsymbol{Y})^{2} \\
& =(\boldsymbol{g} \odot \boldsymbol{g})(\boldsymbol{X}, \boldsymbol{Y}, \boldsymbol{X}, \boldsymbol{Y})
\end{aligned}
$$

Note that $|\boldsymbol{Q}(\boldsymbol{X}, \boldsymbol{Y})|$ gives the square of the area of the parallelogram spanned by $\boldsymbol{X}$ and $\boldsymbol{Y}$ which is nonzero iff the considered plane is spacelike or timelike (nondegenerate).

In (A7) we introduced the product $\odot$, which is called the Kulkarni-Nomizu product. It is a symmetric bilinear map from the space of symmetric $(0,2)$ tensors to the space of $(0,4)$ tensors with the same algebraic symmetries as Riem. Its general definition is as follows:

$$
\begin{aligned}
& (\boldsymbol{a} \odot \boldsymbol{b})(\boldsymbol{W}, \boldsymbol{Z}, \boldsymbol{X}, \boldsymbol{Y}):= \\
& \quad \frac{1}{2}(\boldsymbol{a}(\boldsymbol{W}, \boldsymbol{X}) \boldsymbol{b}(\boldsymbol{Z}, \boldsymbol{Y})-\boldsymbol{a}(\boldsymbol{W}, \boldsymbol{Y}) \boldsymbol{b}(\boldsymbol{Z}, \boldsymbol{X}) \\
& \quad+\boldsymbol{b}(\boldsymbol{W}, \boldsymbol{X}) \boldsymbol{a}(\boldsymbol{Z}, \boldsymbol{Y})-\boldsymbol{b}(\boldsymbol{W}, \boldsymbol{Y}) \boldsymbol{a}(\boldsymbol{Z}, \boldsymbol{X})) .
\end{aligned}
$$

This can be used to conveniently write down the $\boldsymbol{g}$ orthogonal decomposition of the curvature tensor into the Ricci- and the Weyl part:

$$
\text { Riem }=\text { Ricci }+ \text { Weyl . }
$$


In four spacetime dimensions one has

$$
\text { Ricci } \begin{aligned}
: & =\left(\text { Ric }-\frac{1}{6} \text { Scal } \boldsymbol{g}\right) \odot \boldsymbol{g} \\
& =\left(\operatorname{Ein}-\frac{1}{3} \operatorname{tr}_{\boldsymbol{g}}(\text { Ein }) \boldsymbol{g}\right) \odot \boldsymbol{g}
\end{aligned}
$$

Inserting this into (A9) gives the definition of Weyl. The definition is such that the Ricci part is $\boldsymbol{g}$-orthogonal to the Weyl part and that the latter is totally trace free. Hence the Ricci and the Weyl part each contribute 10 independent components to the 20 independent components of Riem. The Ricci part may be further decomposed according to the decomposition of Ric into its trace and a trace-free part, but this refinement will not be needed here.

Einstein's equation now express the local determination of the Ricci part of the curvature in terms of the energy-momentum distribution of matter, the latter being encoded in the energy-momentum tensor $\boldsymbol{T}$ of the matter. In units where Newton's constant $G$ and the velocity of light $c$ equal one ${ }^{20}$, Einstein's equation reads

$$
\operatorname{Ein}=8 \pi \boldsymbol{T} .
$$

Here we did not write down explicitly a cosmological term, which can always be thought of as extra contribution to $\boldsymbol{T}$ of the form $\boldsymbol{g} \Lambda / 8 \pi$. Now, assuming that $\boldsymbol{g}$ satisfies Einstein's equation, the Ricci part of the Riemann tensor is given in terms of $\boldsymbol{T}$ by

$$
\text { Ricci }=8 \pi\left(\boldsymbol{T}-\frac{1}{3} \operatorname{tr}_{\boldsymbol{g}}(\boldsymbol{T}) \boldsymbol{g}\right) \odot \boldsymbol{g}
$$

\section{APPENDIX B: Proof of Theorem 1}

In this section we prove Theorem 1, namely the equivalence, in the spherically-symmetric case, of the SSJC with the Darmois junction conditions.

Proof. The proof essentially consists in writing down the induced metric and extrinsic curvature for a (non-null) spherically symmetric hypersurface in a spherically symmetric spacetime. This is most easily done by introducing an adapted orthonormal frame.

We first consider the case where $\Gamma$ is timelike, hence $\gamma=\pi(\Gamma)$ is a timelike curve in $\mathcal{B}$. The following construction shall be carried out in both spacetimes. One defines $\boldsymbol{v}$ as in the SSJC, hence as the (unique up to a sign) spherically symmetric, unit vector field on $\Gamma$ orthogonal to $\boldsymbol{n}$. That is $\boldsymbol{v}$, seen as a vector field on $\mathcal{B}$, is tangent to $\gamma$. Since $\boldsymbol{n}$ is spacelike, $\boldsymbol{v}$ is timelike. The ambient metric can be then written as

$$
\boldsymbol{g}=\underline{\boldsymbol{v}} \otimes \underline{\boldsymbol{v}}-\underline{\boldsymbol{n}} \otimes \underline{\boldsymbol{n}}-R^{2} \boldsymbol{g}_{S^{2}},
$$

20 Otherwise the factor $8 \pi$ on the right-hand side of A11 should be replaced with $8 \pi G / c^{4}$. so that the induced metric (compare Appendix $\mathrm{C}$ and (C3) on $\Gamma$ is

$$
\boldsymbol{g}_{\Gamma}=\underline{\boldsymbol{v}} \otimes \underline{\boldsymbol{v}}-R^{2} \boldsymbol{g}_{S^{2}}
$$

In view of (C3) note that here $\varepsilon(\boldsymbol{n})=-1$. For the extrinsic curvature (C6), using (D3) and the fact that $\boldsymbol{v}$ is spherically symmetric and hence tangent to $\mathcal{B}$, one has the decomposition

$$
\boldsymbol{K}_{\Gamma}=-\boldsymbol{g}\left(\boldsymbol{n}, \nabla_{\boldsymbol{v}} \boldsymbol{v}\right) \underline{\boldsymbol{v}} \otimes \underline{\boldsymbol{v}}-R \boldsymbol{d} R(\boldsymbol{n}) \boldsymbol{g}_{S^{2}}
$$

Now, from expressions (B2) and (B3) it follows that the DJC, and hence the continuity of $\boldsymbol{g}_{\Gamma}$ and $\boldsymbol{K}_{\Gamma}$, are equivalent to the continuity of the following four functions:

(a) the arc-length of $\gamma$,

(b) $R$,

(c) $\boldsymbol{d} R(\boldsymbol{n})$, and

(d) $\boldsymbol{g}\left(\boldsymbol{n}, \boldsymbol{\nabla}_{\boldsymbol{v}} \boldsymbol{v}\right)$.

The statement of the theorem will now follow from the following expression of the MS energy (58):

$$
E=\frac{R}{2}\left(1+(\boldsymbol{d} R(\boldsymbol{v}))^{2}-(\boldsymbol{d} R(\boldsymbol{n}))^{2}\right) .
$$

Simply note that if $R$ is continuous through $\Gamma$ (recall the definition of this concept below the definition of DJC in Section V.A the same holds for its derivatives tangent to $\Gamma$. In particular, $\boldsymbol{d} R(\boldsymbol{v})$ is continuous through $\Gamma$ and hence we may substitute $\boldsymbol{d} R(\boldsymbol{n})$ by the MS energy in the above list (a)-(d). This completes the proof for timelike $\Gamma$.

In the case of spacelike $\Gamma$ the unit normal $\boldsymbol{n}$ is timelike and $\boldsymbol{v}$ is chosen as the unique (up to a sign) spherically symmetric unit vector field on $\Gamma$ orthonormal to $\boldsymbol{n}$. Then $\boldsymbol{v}$ is a spacelike 'radial' unit vector field orthogonal to the $S O(3)$-orbits. The proof now proceeds analogously to the timelike case. We merely list the expressions for the ambient metric

$$
\boldsymbol{g}=\underline{\boldsymbol{n}} \otimes \underline{\boldsymbol{n}}-\underline{\boldsymbol{v}} \otimes \underline{\boldsymbol{v}}-R^{2} \boldsymbol{g}_{S^{2}}
$$

the induced metric

$$
\boldsymbol{g}_{\Gamma}=\underline{\boldsymbol{v}} \otimes \underline{\boldsymbol{v}}+R^{2} \boldsymbol{g}_{S^{2}}
$$

the extrinsic curvature

$$
\boldsymbol{K}_{\Gamma}=\boldsymbol{g}\left(\boldsymbol{n}, \nabla_{\boldsymbol{v}} \boldsymbol{v}\right) \underline{\boldsymbol{v}} \otimes \underline{\boldsymbol{v}}+R \boldsymbol{d} R(\boldsymbol{n}) \boldsymbol{g}_{S^{2}},
$$

and the MS energy

$$
E=\frac{R}{2}\left(1+(\boldsymbol{d} R(\boldsymbol{n}))^{2}-(\boldsymbol{d} R(\boldsymbol{v}))^{2}\right),
$$

and conclude exactly as in the timelike case. 


\section{APPENDIX C: Submanifolds}

In a Lorentzian manifold $(\mathcal{M}, \boldsymbol{g})$ endowed with LeviCivita connection $\boldsymbol{\nabla}$ consider a smooth submanifold $\Gamma$ of co-dimension one and normal vector field $\boldsymbol{n}$. We assume $\Gamma$ to be non-null, that is, either spacelike (then $\boldsymbol{n}$ is timelike) or timelike (then $\boldsymbol{n}$ spacelike). Then $\Gamma$ inherits from the ambient manifold $\mathcal{M}$ a (non-degenerate) metric and a connection in a natural way. We introduce the orthogonal projectors

$$
\begin{aligned}
& \boldsymbol{Q}_{\boldsymbol{n}}:=\varepsilon(\boldsymbol{n}) \boldsymbol{n} \otimes \underline{\boldsymbol{n}} \\
& \boldsymbol{P}_{\boldsymbol{n}}:=\mathrm{id}-\boldsymbol{Q}_{\boldsymbol{n}},
\end{aligned}
$$

where $\varepsilon(\boldsymbol{n})$ denotes the indicator, defined for any nonnull vector by

$$
\varepsilon(\boldsymbol{X}):=\frac{\boldsymbol{g}(\boldsymbol{X}, \boldsymbol{X})}{|\boldsymbol{g}(\boldsymbol{X}, \boldsymbol{X})|}= \begin{cases}+1 & \text { if } \boldsymbol{X} \text { is timelike } \\ -1 & \text { if } \boldsymbol{X} \text { is spacelike }\end{cases}
$$

The induced metric on $\Gamma$ (also called first fundamental form) is given by

$$
\boldsymbol{g}_{\Gamma}:=-\varepsilon(\boldsymbol{n}) \boldsymbol{P}_{\boldsymbol{n}} \boldsymbol{g}
$$

where the sign is just in order to get a positive definite metric in the case where $\Gamma$ is spacelike. Given two vector fields $\boldsymbol{X}, \boldsymbol{Y}$ tangent to $\Gamma$, so that $\boldsymbol{Q}_{\boldsymbol{n}} \boldsymbol{X}=\boldsymbol{Q}_{\boldsymbol{n}} \boldsymbol{Y}=0$, one may decompose the covariant derivative of $\boldsymbol{Y}$ with respect to $\boldsymbol{X}$ into its orthogonal components

$$
\begin{aligned}
\boldsymbol{\nabla}_{\boldsymbol{X}} \boldsymbol{Y} & =\boldsymbol{P}_{\boldsymbol{n}}\left(\boldsymbol{\nabla}_{\boldsymbol{X}} \boldsymbol{Y}\right)+\boldsymbol{Q}_{\boldsymbol{n}}\left(\boldsymbol{\nabla}_{\boldsymbol{X}} \boldsymbol{Y}\right) \\
& ={ }^{\Gamma} \boldsymbol{\nabla}_{\boldsymbol{X}} \boldsymbol{Y}+\boldsymbol{K}_{\Gamma}(\boldsymbol{X}, \boldsymbol{Y}) \boldsymbol{n}
\end{aligned}
$$

where

$$
{ }^{\Gamma} \nabla_{\boldsymbol{X}} \boldsymbol{Y}:=\boldsymbol{P}_{\boldsymbol{n}}\left(\boldsymbol{\nabla}_{\boldsymbol{X}} \boldsymbol{Y}\right)
$$

is the induced connection on $\Gamma$ and

$$
\begin{aligned}
\boldsymbol{K}_{\Gamma}(\boldsymbol{X}, \boldsymbol{Y}): & =\varepsilon(\boldsymbol{n}) \boldsymbol{g}\left(\boldsymbol{\nabla}_{\boldsymbol{X}} \boldsymbol{Y}, \boldsymbol{n}\right) \\
& =-\varepsilon(\boldsymbol{n}) \boldsymbol{g}\left(\boldsymbol{\nabla}_{\boldsymbol{X}} \boldsymbol{n}, \boldsymbol{Y}\right)
\end{aligned}
$$

is the extrinsic curvature of $\Gamma$ in $\mathcal{M}$ (also called the second fundamental form). The second equality sign in (C6) is an immediate consequence of the metricity of $\boldsymbol{\nabla}$ and the fact that $\boldsymbol{X}$ and $\boldsymbol{Y}$ are orthogonal to $\boldsymbol{n}$. With this alternative expression for the extrinsic curvature one has $\boldsymbol{K}_{\Gamma}=-\varepsilon(\boldsymbol{n}) \boldsymbol{P}_{\boldsymbol{n}} \boldsymbol{\nabla} \underline{\boldsymbol{n}}$ and hence ${ }^{21}$

$$
\boldsymbol{K}_{\Gamma}=-\varepsilon(\boldsymbol{n}) \mathcal{S}\left(\boldsymbol{P}_{\boldsymbol{n}} \boldsymbol{\nabla} \underline{\boldsymbol{n}}\right) .
$$

Since $\boldsymbol{n}$ is hypersurface orthogonal (by definition) we have $\mathcal{A}\left(\boldsymbol{P}_{\boldsymbol{n}} \boldsymbol{\nabla} \underline{\boldsymbol{n}}\right)=0$. Hence, the extrinsic curvature is a

\footnotetext{
${ }^{21}$ Here and below $\mathcal{S}$ and $\mathcal{A}$ denote the projection operators of full symmetrization and full antisymmetrization, respectively.
}

symmetric $(0,2)$-tensor field. We recall also that the induced connection is the Levi-Civita connection of $\left(\Gamma, \boldsymbol{g}_{\Gamma}\right)$, as one may easily check.

The full relations between the curvature of $\mathcal{M}$ and those (intrinsic and extrinsic) of $\Gamma$ can be found, e.g., in (Giulini, 1998). Here we are only interested in the 'Einstein part' of the curvature. One gets:

$$
\begin{gathered}
\operatorname{Ein}(\boldsymbol{n}, \boldsymbol{n})=\frac{1}{2}\left(-\varepsilon(\boldsymbol{n})^{\Gamma} \mathrm{Scal}+(\operatorname{tr} \boldsymbol{K})^{2}-\|\boldsymbol{K}\|^{2}\right) \\
\operatorname{Ein}\left(\boldsymbol{n}, \boldsymbol{P}_{\boldsymbol{n}} \cdot\right)=-\varepsilon(\boldsymbol{n}) \operatorname{div}_{\Gamma}\left(\boldsymbol{K}-(\operatorname{tr} \boldsymbol{K}) \boldsymbol{g}_{\Gamma}\right) \\
\operatorname{Ein}\left(\boldsymbol{P}_{\boldsymbol{n}} \cdot, \boldsymbol{P}_{\boldsymbol{n}} \cdot\right)={ }^{\Gamma} \operatorname{Ein} \\
+\varepsilon(\boldsymbol{n})\left(\frac{1}{2}\left((\operatorname{tr} \boldsymbol{K})^{2}+\|\boldsymbol{K}\|^{2}\right) \boldsymbol{g}_{\Gamma}-(\operatorname{tr} \boldsymbol{K}) \boldsymbol{K}\right. \\
\left.+\mathbf{L}_{\boldsymbol{n}}\left(\boldsymbol{K}-(\operatorname{tr} \boldsymbol{K}) \boldsymbol{g}_{\Gamma}\right)\right) .
\end{gathered}
$$

\section{APPENDIX D: Spherical symmetry}

We recall that the isometry group, $\operatorname{Isom}(\mathcal{M}, \boldsymbol{g})$, of a spacetime $(\mathcal{M}, \boldsymbol{g})$ is the subgroup of the diffeomorphism group of $\mathcal{M}, \operatorname{Diff}(\mathcal{M})$, which leaves the metric $\boldsymbol{g}$ invariant: $\operatorname{Isom}(\mathcal{M}, \boldsymbol{g}):=\left\{\phi \in \operatorname{Diff}(\mathcal{M}) \mid \phi^{*} \boldsymbol{g}=\boldsymbol{g}\right\}$.

Definition 3 (Spherical symmetry). A four-dimensional Lorentzian manifold $(\mathcal{M}, \boldsymbol{g})$ is said to be spherically symmetric if its isometry group, $\operatorname{Isom}(\mathcal{M}, \boldsymbol{g})$, contains a subgroup $G$ with the following two properties: $(i) G$ is isomorphic to $S O(3)$ and ( $i i)$ each orbit of $G$ is spacelike and two-dimensional (up to some closed proper subset of fixed points). A tensor field $\boldsymbol{T}$ on a spherically symmetric spacetime is said to be spherically symmetric if it is invariant under $G$, hence if $\phi^{*} \boldsymbol{T}=\boldsymbol{T}$ for all $\phi \in G$.

From this definition it follows (excluding the case where the orbits of $G$ are diffeomorphic to the two-dimensional real-projective space) that a fourdimensional spherically symmetric Lorentzian manifold $(\mathcal{M}, \boldsymbol{g})$ can, at least locally, be expressed as a warped product $\mathcal{M}=\mathcal{B} \times_{R} S^{2}$ between a two-dimensional Lorentzian manifold $\left(\mathcal{B}, \boldsymbol{g}_{\mathcal{B}}\right)$, called the 'base', and the standard unit two-sphere $\left(S^{2}, \boldsymbol{g}_{S^{2}}\right)$, called the 'fiber' (see (Straumann, 2004) and (O’Neill, 1983)). This means that, at least locally, the manifold is a product

$$
\mathcal{M} \stackrel{\text { loc }}{=} \mathcal{B} \times S^{2}
$$

and the metric is given by

$$
\boldsymbol{g}=\pi^{*}\left(\boldsymbol{g}_{\mathcal{B}}\right)-(R \circ \pi)^{2} \sigma^{*}\left(\boldsymbol{g}_{S^{2}}\right) .
$$

Here, $\pi$ and $\sigma$ are the projections of $\mathcal{B} \times S^{2}$ onto $\mathcal{B}$ and $S^{2}$, respectively, and $\pi^{*}, \sigma^{*}$ their pull-backs. The warping function $R$ is nothing but the areal radius, since, for a point $p \in \mathcal{B}$, the area of the fiber $p \times S^{2}$ is just $4 \pi R(p)^{2}$.

In this contest, a vector field $\boldsymbol{X}$ on $\mathcal{M}$ at some point $(p, q) \in \mathcal{B} \times S^{2}$ has then a unique decomposition $\boldsymbol{X}=$ 
$\tan _{\mathcal{B}} \boldsymbol{X}+\tan _{S^{2}} \boldsymbol{X}$ in a component tangent to the 'leaves' $\mathcal{B} \times q=\sigma^{-1}(q)$ and a component tangent to the 'fibers' $p \times S^{2}=\pi^{-1}(p)$. Arbitrary tensor fields on $\mathcal{B}$ and on $S^{2}$ can be lifted to tensor fields on $\mathcal{M}$ in the standard way. For covariant tensor fields (and hence, in particular, for functions) this is achieved via the pull-pack of the respective projection: as an example, just look at (D2). For contravariant tensor fields it suffices to consider the special case of vector fields. Let, for instance, $\boldsymbol{X}$ be a vector in the tangent space of $\mathcal{B}$ at $p$. Then the lift $\tilde{\boldsymbol{X}}$ at $(p, q)$ of $\boldsymbol{X}$ is defined as the unique vector in the tangent space of $\mathcal{M}$ at $(p, q)$ with $\pi_{*}(\tilde{\boldsymbol{X}})=\boldsymbol{X}$ and $\sigma_{*}(\tilde{\boldsymbol{X}})=0$. Since this assignment is smooth, one gets the lifting of a vector field via the pointwise lifting just described. In this work, we will mainly omit lifts and projections and not explicitly distinguish between original and lifted quantities. For example, when referring to a vector 'tangent to $\mathcal{B}$ ' we refer to a vector in the tangent space of $\mathcal{B}$ or to the lift thereof in the tangent space of $\mathcal{M}$.

If $\boldsymbol{X}$ is spherically symmetric, then the component tangent to the fibers must vanish: $\tan _{S^{2}} \boldsymbol{X}=0$. Similarly, a spherically symmetric one-form $\boldsymbol{\theta}$ on $\mathcal{M}$ must necessarily be tangent to $\mathcal{B}$ (i.e. normal to $S^{2}$ ) and thus it can be written as $\boldsymbol{\theta}=\pi^{*}\left(\boldsymbol{\theta}_{\mathcal{B}}\right)$, where $\boldsymbol{\theta}_{\mathcal{B}}$ is a one-form on $\mathcal{B}$. Finally, a spherical symmetric function is simply the lift of a function on $\mathcal{B}$.

\section{Connection and curvature decomposition}

In the following we discuss relations which express the curvature of $\mathcal{M}$ in terms of the warping function $R$ and the curvatures of the base $\mathcal{B}$ and the fiber $S^{2}$. We start out from the relations between the Levi-Civita connection $\boldsymbol{\nabla}$ of $(\mathcal{M}, \boldsymbol{g})$ and the Levi-Civita connections of the base and the fiber, denoted by ${ }^{\mathcal{B}} \nabla$ and ${ }^{S^{2}} \nabla$, respectively. These relations can be derived, for example, by means of the Koszul formula (see e.g. (O'Neill, 1983) Proposition 7.35). Let in the following $\boldsymbol{X}, \boldsymbol{Y}, \boldsymbol{Z}$ be vector fields tangent to $\mathcal{B}$ and $\boldsymbol{U}, \boldsymbol{V}, \boldsymbol{W}$ tangent to $S^{2}$. Suppressing lifts and projections, we have:

$$
\begin{aligned}
& \nabla_{\boldsymbol{X}} \boldsymbol{Y}={ }^{\mathcal{B}} \boldsymbol{\nabla}_{\boldsymbol{X}} \boldsymbol{Y} \\
& \boldsymbol{\nabla}_{\boldsymbol{X}} \boldsymbol{V}=\boldsymbol{\nabla}_{\boldsymbol{V}} \boldsymbol{X}=R^{-1} \boldsymbol{X}(R) \boldsymbol{V} \\
& \tan _{S^{2}} \boldsymbol{\nabla}_{\boldsymbol{V}} \boldsymbol{W}={ }^{S^{2}} \boldsymbol{\nabla}_{\boldsymbol{V}} \boldsymbol{W} \\
& \tan _{\mathcal{B}} \boldsymbol{\nabla}_{\boldsymbol{V}} \boldsymbol{W}=-\boldsymbol{g}(\boldsymbol{V}, \boldsymbol{W}) R^{-1} \boldsymbol{\nabla} R .
\end{aligned}
$$

Note that, for a function $f$ on $\mathcal{B}$, the lift of the gradient is equal to the gradient of the lifted function, that is (suppressing the lifts): $\operatorname{grad} f={ }^{\mathcal{B}} \operatorname{grad} f$. For brevity, we write just grad $f$ for it. Take care that for the Hessian and the Laplacian this is in general not true (see (D13) and (D14)). Therefore we write explicitly the superscripts ' $\mathcal{B}$ ' in ${ }^{\mathcal{B}}$ Hess $f$ and ${ }^{\mathcal{B}} \Delta f$ to denote the Hessian and Laplacian of $f$ on $\mathcal{B}$, respectively, or the lifts thereof.

By means of (D3) one can now compute the expressions for the Riemann tensor. As the sectional curvature of $S^{2}$ is obviously constant and equal to one, the Riemann tensor, the Ricci tensor, and the Ricci scalar of $S^{2}$ are simply given by ${ }^{S^{2}} \mathbf{R i e m}=\boldsymbol{g}_{S^{2}} \odot \boldsymbol{g}_{S^{2}},{ }^{S^{2}} \mathbf{R i c}=\boldsymbol{g}_{S^{2}}$, and ${ }^{S^{2}}$ Scal $=2$, respectively. Here we made again use of the Kulkarni-Nomizu product (A8). Moreover, since the basis manifold $\mathcal{B}$ is two-dimensional, one can express its curvature tensors in terms of the scalar curvature. The expression for the Riemann tensor, Ricci tensor, and Ricci scalar of a spherically symmetric Lorentzian manifold (D1|D2) are, respectively,

$$
\begin{aligned}
\text { Riem } & =\frac{{ }^{\mathcal{B}} \mathrm{Scal}}{2} \boldsymbol{g}_{\mathcal{B}} \odot \boldsymbol{g}_{\mathcal{B}} \\
& -\frac{1}{R^{2}}(1+\langle\boldsymbol{d} R, \boldsymbol{d} R\rangle) R^{2} \boldsymbol{g}_{S^{2}} \odot R^{2} \boldsymbol{g}_{S^{2}} \\
& +2 R \boldsymbol{g}_{S^{2}} \odot \text { Hess } R \\
\text { Ric } & =\frac{{ }^{\mathcal{B}} \mathrm{Scal}}{2} \boldsymbol{g}_{\mathcal{B}}-\frac{2}{R}{ }^{\mathcal{B}} \text { Hess } R \\
& +\left(1+\langle\boldsymbol{d} R, \boldsymbol{d} R\rangle+R{ }^{\mathcal{B}} \Delta R\right) \boldsymbol{g}_{S^{2}}
\end{aligned}
$$

and

$$
\text { Scal }={ }^{\mathcal{B}} \text { Scal }-\frac{2}{R^{2}}(1+\langle\boldsymbol{d} R, \boldsymbol{d} R\rangle)-\frac{4}{R}{ }^{\mathcal{B}} \Delta R .
$$

Hence, for the Einstein tensor we have the expression

$$
\begin{aligned}
\operatorname{Ein}= & \left(\frac{1}{R^{2}}(1+\langle\boldsymbol{d} R, \boldsymbol{d} R\rangle)+\frac{2}{R}{ }^{\mathcal{B}} \Delta R\right) \boldsymbol{g}_{\mathcal{B}}-\frac{2}{R}{ }^{\mathcal{B}} \text { Hess } R \\
& +\left(\frac{{ }^{\mathcal{B}} \mathrm{Scal}}{2}-\frac{1}{R}{ }^{\mathcal{B}} \Delta R\right) R^{2} \boldsymbol{g}_{S^{2}}
\end{aligned}
$$

and for the Weyl tensor, using (A9) and (A10a), the simple expression

$$
\text { Weyl }=w\left(\boldsymbol{g}_{\mathcal{B}} \odot \boldsymbol{g}_{\mathcal{B}}+\boldsymbol{g}_{\mathcal{B}} \odot R^{2} \boldsymbol{g}_{S^{2}}+R^{2} \boldsymbol{g}_{S^{2}} \odot R^{2} \boldsymbol{g}_{S^{2}}\right) .
$$

Here we put

$$
\begin{aligned}
w & :=\frac{1}{6}\left({ }^{\mathcal{B}} \text { Scal }-\frac{2}{R^{2}}(1+\langle\boldsymbol{d} R, \boldsymbol{d} R\rangle)+\frac{2}{R}{ }^{\mathcal{B}} \Delta R\right) \\
& =\frac{1}{6} \mathrm{Scal}+\frac{1}{R}{ }^{\mathcal{B}} \Delta R .
\end{aligned}
$$

In the derivation of (D8) we made use of the formula $\boldsymbol{h}_{\mathcal{B}} \odot \boldsymbol{g}_{\mathcal{B}}=(1 / 2)\left(\operatorname{tr}_{\boldsymbol{g}_{\mathcal{B}}} \boldsymbol{h}_{\mathcal{B}}\right) \boldsymbol{g}_{\mathcal{B}} \odot \boldsymbol{g}_{\mathcal{B}}$, valid ${ }^{22}$ for any symmetric bilinear form $\boldsymbol{h}_{\mathcal{B}}$ on $\mathcal{B}$, in order to express the only term involving ${ }^{\mathcal{B}}$ Hess $R$ in terms of the Laplacian of $R$. Note that from (D8a) it is immediate that the Weyl tensor of a spherically symmetric spacetime has only one

\footnotetext{
22 To prove this just note that the only independent component of this formula is the $\left(\boldsymbol{e}_{0}, \boldsymbol{e}_{1}, \boldsymbol{e}_{0}, \boldsymbol{e}_{1}\right)$ one, where $\left\{\boldsymbol{e}_{\mu}\right\}$ is an adapted orthonormal basis of $(\mathcal{M}, \boldsymbol{g})$ such that $\boldsymbol{e}_{0}, \boldsymbol{e}_{1}$ are tangent to $\mathcal{B}$ and $\boldsymbol{e}_{2}, \boldsymbol{e}_{3}$ are tangent to $S^{2}$. Then, the equality follows immediately using the definition A8 of the Kulkarni-Nomizu product.
} 
independent component, as it must be the case due to it being of Petrov-type D.

Comparing expression (D4) with the definition of sectional curvature (A6) one can immediately read off that the sectional curvature $K$ of the plane tangential to the (two-dimensional) $S O(3)$-orbits at a given point is

$$
K=-\frac{1}{R^{2}}(1+\langle\boldsymbol{d} R, \boldsymbol{d} R\rangle)
$$

and hence the MS energy, defined as (minus one-half) $K$ times the third power of the areal radius, is given by (58). Using the MS energy (58), we can write the Einstein tensor (D7) as:

$$
\begin{aligned}
\text { Ein }= & \frac{2}{R}\left({ }^{\mathcal{B}} \Delta R+\frac{E}{R^{2}}\right) \boldsymbol{g}_{\mathcal{B}}-\frac{2}{R}{ }^{\mathcal{B}} \text { Hess } R \\
& +\left(\frac{{ }^{\mathcal{B}} \text { Scal }}{2}-\frac{1}{R}{ }^{\mathcal{B}} \Delta R\right) R^{2} \boldsymbol{g}_{S^{2}} .
\end{aligned}
$$

We conclude giving the decomposition for the divergence of a spherically symmetric vector field (that is a vector field $\boldsymbol{X}$ tangent to $\mathcal{B}$ ) and for the Hessian and Laplacian of a spherically symmetric function (that is a function $f$ on $\mathcal{B}$ ). First, from (D3a) and (D3b), we obtain the following decomposition for the covariant derivative of $\boldsymbol{X}$ (expressed as a $(0,2)$-tensor):

$$
\boldsymbol{\nabla} \underline{\boldsymbol{X}}={ }^{\mathcal{B}} \boldsymbol{\nabla} \underline{\boldsymbol{X}}-\boldsymbol{X}(R) R \boldsymbol{g}_{S^{2}}
$$

Note that the mixed term $\left(\mathcal{B}-S^{2}\right)$ vanishes - as it should due to spherical symmetry. Taking the trace of (D11) one obtains the following expression for the divergence:

$$
\operatorname{div} \boldsymbol{X}=\operatorname{div}_{\mathcal{B}} \boldsymbol{X}+\frac{2}{R} \boldsymbol{X}(R)=\frac{1}{R^{2}} \operatorname{div}_{\mathcal{B}}\left(R^{2} \boldsymbol{X}\right)
$$

The decompositions for the Hessian and Laplacian of a function $f$ on $\mathcal{B}$ follow from inserting $\boldsymbol{X}=\operatorname{grad} f$ in the above formulae. One gets:

$$
\text { Hess } f={ }^{\mathcal{B}} \text { Hess } f-\boldsymbol{g}_{\mathcal{B}}(\operatorname{grad} f, \operatorname{grad} R) R \boldsymbol{g}_{S^{2}}
$$

and

$$
\Delta f={ }^{\mathcal{B}} \Delta f+2 \boldsymbol{g}_{\mathcal{B}}(\operatorname{grad} f, \operatorname{grad} R) / R,
$$

respectively.

\section{Einstein equation in case of spherical symmetry}

A general spherically symmetric matter energymomentum tensor has the form

$$
\boldsymbol{T}=\boldsymbol{T}_{\mathcal{B}}+p R^{2} \boldsymbol{g}_{S^{2}},
$$

where $p$ is the spherical part of the pressure. Hence, using the decomposition (D10) of the Einstein's tensor found in Appendix D, the Einstein equation takes the form

$$
\begin{aligned}
& \frac{2}{R}\left(\frac{E}{R^{2}}+{ }^{\mathcal{B}} \Delta R\right) \boldsymbol{g}_{\mathcal{B}}-\frac{2}{R}{ }^{\mathcal{B}} \text { Hess } R=8 \pi \boldsymbol{T}_{\mathcal{B}} \\
& \frac{{ }^{\mathcal{B}} \text { Scal }}{2}-\frac{1}{R}{ }^{\mathcal{B}} \Delta R=8 \pi p .
\end{aligned}
$$

Using the trace of the first equation,

$$
\frac{1}{R}\left({ }^{\mathcal{B}} \Delta R+\frac{2 E}{R^{2}}\right)=4 \pi \operatorname{tr} \boldsymbol{T}_{\mathcal{B}},
$$

to eliminate ${ }^{\mathcal{B}} \Delta R$, one can write (D16) in the equivalent form

$$
\begin{aligned}
& \frac{1}{R}\left(\frac{E}{R^{2}} \boldsymbol{g}_{\mathcal{B}}+{ }^{\mathcal{B}} \text { Hess } R\right)=-4 \pi \star \boldsymbol{T}_{\mathcal{B} \star} \\
& \frac{{ }^{\mathcal{B}} \mathrm{Scal}}{2}+\frac{2 E}{R^{2}}=4 \pi\left(\operatorname{tr} \boldsymbol{T}_{\mathcal{B}}+2 p\right) .
\end{aligned}
$$

Here, and in the following, $\star$ denotes the Hodge-duality map for $\left(\mathcal{B}, \boldsymbol{g}_{\mathcal{B}}\right)$ (for the definition, see e.g. (Straumann, 2004)). In the first equation we used the identity $\star \tau \star=$ $\boldsymbol{\tau}-\operatorname{tr}(\boldsymbol{\tau}) \boldsymbol{g}$, which is valid for any bilinear form $\boldsymbol{\tau}$ on $\mathcal{B}$, where the first (second) star acts on the first (second) slot of $\tau$.

Finally, the integrability condition $\operatorname{div} \boldsymbol{T}=0$ for the energy-momentum tensor (D15) reads

$$
\operatorname{div}_{\mathcal{B}}\left(R^{2} \boldsymbol{T}_{\mathcal{B}}\right)+p \boldsymbol{d}\left(R^{2}\right)=0 .
$$

\section{Misner-Sharp energy}

Let us now turn to the MS energy and its properties. We first show that it is the charge of a conserved current. The treatment presented here follows mainly (Hayward, 1996). In a spherically symmetric spacetime one defines the Kodama vector field (Kodama, 1980) as the (unique up to a sign) spherically symmetric vector field orthogonal to, and of the same norm as, the gradient of $R$; hence we put

$$
\underline{\boldsymbol{k}}:=\star \boldsymbol{d} R \text {. }
$$

With this sign choice $\boldsymbol{k}$ is future-pointing if the gradient of $R$ is spacelike. The orthogonality between $k$ and the gradient of $R$ is simply expressed by

$$
\boldsymbol{k}(R)=0,
$$

which clearly means that the integral curves of $\boldsymbol{k}$ stay at constant areal radius.

An immediate but important property of the Kodama vector field is that it is conserved:

$$
\operatorname{div} k=0 .
$$

Indeed, using (D12) and (D21), one has: $\operatorname{div} \boldsymbol{k}=$ $\operatorname{div}_{\mathcal{B}} \boldsymbol{k}=\boldsymbol{\delta} \underline{\boldsymbol{k}}=-\star \boldsymbol{d} \star \star \boldsymbol{d} R \equiv 0$.

Now, a key point for the study of spherically symmetric spacetimes is the following equation relating the MS energy with the matter's energy-momentum tensor:

$$
\boldsymbol{d} E=4 \pi R^{2} \star \underline{\boldsymbol{j}}
$$

where $\boldsymbol{j}$ is the so-called Kodama current (tangent to the base manifold $\mathcal{B}$ ) defined by

$$
\underline{j}:=T(k, \cdot) .
$$


Equation (D23) follows from Einstein's equation; more precisely, it is equivalent to its $\mathcal{B}$-part (that is Eq. (D18a)) fed with $\nabla R$. To see this, just compute the differential of (58) as follows: $\boldsymbol{d} E=(E / R) \boldsymbol{d} R+(R / 2) \boldsymbol{d}\langle\boldsymbol{d} R, \boldsymbol{d} R\rangle=$ $(E / R) \boldsymbol{d} R+R^{\mathcal{B}}$ Hess $R \cdot \boldsymbol{d} R=R\left(\left(E / R^{2}\right) \boldsymbol{g}_{\mathcal{B}}+{ }^{\mathcal{B}}\right.$ Hess $\left.R\right) \cdot$ $\boldsymbol{d} R$, where the dot denotes here the contraction of the last slot of the tensor on the left with the first slot of the tensor on the right of the dot. In the second step we use that $\boldsymbol{d}(\langle\boldsymbol{d} R, \boldsymbol{d} R\rangle)(\boldsymbol{X})=\boldsymbol{X}(\boldsymbol{g}(\boldsymbol{\nabla} R, \boldsymbol{\nabla} R))=$ $2 \boldsymbol{g}_{\mathcal{B}}\left(\nabla_{\boldsymbol{X}} \boldsymbol{\nabla} R, \boldsymbol{\nabla} R\right)=2{ }^{\mathcal{B}}$ Hess $R(\boldsymbol{\nabla} R, \boldsymbol{X})$ for any $\boldsymbol{X}$ tangent to $\mathcal{B}$. Then, inserting (D18a) and using that * is skew-adjoint on one-forms, one gets $\boldsymbol{d} E=-4 \pi R^{2} \star$ $\boldsymbol{T}_{\mathcal{B}} \star \cdot \boldsymbol{d} R=4 \pi R^{2} \star \boldsymbol{T}_{\mathcal{B}} \cdot \star \boldsymbol{d} R$ and hence, using the definitions (D20) and (D24) together with the symmetry of $\boldsymbol{T}$, one arrives at (D23).

From (D23) it is clear that

$$
\boldsymbol{j}(E)=0,
$$

which means that the vector field $\boldsymbol{j}$ is tangent to the curves in $\mathcal{B}$ (hypersurfaces in $\mathcal{M}$ ) of constant MS energy. Moreover, (D23) implies that $\boldsymbol{j}$ is also conserved

$$
\operatorname{div} \boldsymbol{j}=0,
$$

where the divergence is here taken on the spacetime $(\mathcal{M}, \boldsymbol{g})$. To see this, just compute the divergence of $\boldsymbol{j}$ with (D12) and using the Hodge-dual version of (D23): $\operatorname{div} \boldsymbol{j}=R^{-2} \operatorname{div}_{\mathcal{B}}\left(R^{2} \boldsymbol{j}\right)=R^{-2} \boldsymbol{\delta}\left(R^{2} \underline{\boldsymbol{j}}\right)=\left(4 \pi R^{2}\right)^{-1} \boldsymbol{\delta} \star$ $\boldsymbol{d} E \equiv 0$.

Following (Hayward, 1996), we can now show that the charges corresponding to the conserved currents $\boldsymbol{j}$ and $\boldsymbol{k}$ are, respectively, the MS energy and the areal volume. Let $\Sigma$ be some spatial three-dimensional hypersurface which, because of spherical symmetry, decomposes as $\Sigma=\sigma \times S^{2}$, where $\sigma$ is some spatial curve in $\mathcal{B}$. Recall that the charge related to a conserved current $\boldsymbol{X}$ is given by $Q_{\boldsymbol{X}}(\Sigma):=\int_{\Sigma} i_{\boldsymbol{X}} \boldsymbol{\mu}$, where $\boldsymbol{\mu}$ is the volume form on $\mathcal{M}$, and, because of spherical symmetry, the latter decomposes as $\boldsymbol{\mu}=\boldsymbol{\mu}_{\mathcal{B}} \wedge R^{2} \boldsymbol{\mu}_{S^{2}}$, where $\boldsymbol{\mu}_{\mathcal{B}}$ and $\boldsymbol{\mu}_{S^{2}}$ are the volume forms on $\mathcal{B}$ and on the unit two-sphere, respectively. After integration of the spherical part and since $i_{\boldsymbol{j}} \boldsymbol{\mu}_{\mathcal{B}}=\star \underline{\boldsymbol{j}}$, using (D23) one gets

$$
Q_{\boldsymbol{j}}(\Sigma)=\int_{\sigma} \boldsymbol{d} E,
$$

which means that the charge of $\boldsymbol{j}$ is the MS energy. This justifies the interpretation of the MS energy as a quantity associated to the 'interior' of the considered sphere of symmetry. In fact, due to (D26), the charge does not depend how one choose the spatial slice to define the interior. Similarly, since $i_{\boldsymbol{k}} \boldsymbol{\mu}_{\mathcal{B}}=\star \underline{\boldsymbol{k}}=\boldsymbol{d} R$, the charge to $\boldsymbol{k}$ is simply $\int_{\sigma} 4 \pi R^{2} \boldsymbol{d} R$ and hence

$$
Q_{\boldsymbol{k}}(\Sigma)=\int_{\sigma} \boldsymbol{d}\left(\frac{4 \pi}{3} R^{3}\right)
$$

which says that the charge of $\boldsymbol{k}$ is the flat-space volume computed with the areal radius.
Incidentally, the Kodama vector can be used to give an elegant proof of Birkhoff's theorem, which states that spherically symmetric solutions of Einstein's equations are, in fact, static. Indeed, by direct computation one shows that in vacuum $\boldsymbol{k}$ is Killing and, because of spherical symmetry, it is clearly also hypersurface orthogonal.

Next we turn to the relation between the MS energy and the Hawking quasi-local mass (Hawking, 1968). The latter is a quantity associated to a spatial two-sphere, $S$, in an arbitrary spacetime. It is defined by

$$
M_{\mathrm{H}}(S):=\sqrt{\frac{\operatorname{Area}(S)}{16 \pi}}\left(1+\frac{1}{2 \pi} \int_{S} \theta^{+} \theta^{-} \boldsymbol{\mu}_{S}\right) .
$$

Here, $\theta^{ \pm}:=\operatorname{tr}_{S^{2}}\left(\boldsymbol{\nabla} \boldsymbol{l}^{ \pm}\right) / 2$ are, respectively, the expansions of the outgoing and ingoing future-pointing null vector fields $\boldsymbol{l}^{ \pm}$normal to $S$, the latter being partially normalized such that $\boldsymbol{g}\left(\boldsymbol{l}^{+}, \boldsymbol{l}^{-}\right)=1$ (there remains the freedom to rescale $\boldsymbol{l}^{ \pm} \rightarrow \alpha^{ \pm 1} \boldsymbol{l}^{ \pm}$, where $\alpha$ is a positive real-valued function). In the special case of spherical symmetry we take $S$ to be an orbit of the rotation group. Then we clearly have $\operatorname{Area}(S)=4 \pi R^{2}$. It is also obvious that the metric of the base $\mathcal{B}$, evaluated on $S$, can simply be written in the form

$$
\boldsymbol{g}_{\mathcal{B}}=\underline{\boldsymbol{l}}^{+} \otimes \underline{\boldsymbol{l}}^{-}+\underline{\boldsymbol{l}}^{-} \otimes \underline{\mathbf{l}}^{+} .
$$

Now, for $\boldsymbol{V}$ tangent to $S$, (D3b) gives $\nabla_{\boldsymbol{V}} \boldsymbol{l}^{ \pm}=$ $R^{-1} \boldsymbol{l}^{ \pm}(R) \boldsymbol{V}$ so that $\theta^{ \pm}=R^{-1} \boldsymbol{l}^{ \pm}(R)$. Hence we have:

$$
2 \theta^{+} \theta^{-}=2 R^{-2} \boldsymbol{d} R\left(\boldsymbol{l}^{+}\right) \boldsymbol{d} R\left(\boldsymbol{l}^{-}\right)=\langle\boldsymbol{d} R, \boldsymbol{d} R\rangle / R^{2},
$$

where we used (D30), or rather its contravariant version, in the last step. Equation (58) now establishes the equality between the MS energy at $p$ and the Hawking quasilocal mass of $S$, where $p$ is any point on $S$ :

$$
E(p)=M_{\mathrm{H}}(S) .
$$

As is the case for the Hawking quasi-local mass, the MS energy can be naturally decomposed into a Ricci and a Weyl part:

$$
E=E_{\mathrm{R}}+E_{\mathrm{W}},
$$

where

$$
\begin{aligned}
& E_{\mathrm{R}}:=-\frac{1}{2} R^{3} K_{\mathrm{R}}, \\
& E_{\mathrm{W}}:=-\frac{1}{2} R^{3} K_{\mathrm{W}} .
\end{aligned}
$$

Here $K_{\mathrm{R}}$ and $K_{\mathrm{W}}$ denote, respectively, the Ricci and the Weyl parts of the sectional curvature of the plane tangential to the $S O(3)$-orbits. These are obtained inserting the decomposition of the Riemann tensor (A9) in the definition of the sectional curvature (A6). The Ricci part of the MS energy is determined by the local matter distribution via Einstein's equation: Using expressions (D15) and (D2) for an arbitrary spherically symmetric energymomentum tensor and, respectively, metric in (A12) one gets

$$
E_{\mathrm{R}}=\frac{4 \pi}{3} R^{3}\left(\operatorname{tr} \boldsymbol{T}_{\mathcal{B}}+p\right)
$$


For the Weyl part of the MS energy we have, in view of (D8a), that

$$
E_{\mathrm{w}}=-\frac{1}{2} R^{3} w,
$$

where $w$ is given by (D8b) or (D8c). Hence, in particular, the Weyl tensor vanishes iff $E_{\mathrm{W}}$ does. Since the square of the Weyl tensor is $\langle$ Weyl, Weyl $\rangle \equiv W_{\alpha \beta \gamma \delta} W^{\alpha \beta \gamma \delta}=$ $12 w^{2}$, with (D36) we obtain the nice expression

$$
\langle\text { Weyl, Weyl }\rangle=48 \frac{E_{\mathrm{w}}^{2}}{R^{6}} .
$$

From this one sees that, in a spherically-symmetric spacetime, the non-vanishing of $E_{\mathrm{w}}$ (that is the non-vanishing of the Weyl tensor) for $R \rightarrow 0$ implies a curvature singularity at $R=0$.

To gain a better physical understanding of the Weyl part of the MS energy we take a look at the equation of geodesic deviation (sometime called Jacobi equation). Let $\boldsymbol{u}$ be a geodesic observer field and $s$ the spatial $(\boldsymbol{g}(\boldsymbol{u}, \boldsymbol{s})=0)$ separation vector between two nearby integral curves of $\boldsymbol{u}$. Then the equation of geodesic deviation (see e.g. (Straumann, 2004)) is $\boldsymbol{\nabla}_{\boldsymbol{u}} \boldsymbol{\nabla}_{\boldsymbol{u}} \boldsymbol{s}=\boldsymbol{R}(\boldsymbol{u}, \boldsymbol{s}) \boldsymbol{u}=$ $\boldsymbol{B}_{\boldsymbol{u}}(\boldsymbol{s})+\boldsymbol{C}_{\boldsymbol{u}}(\boldsymbol{s})$. In the last step we decomposed the endomorphism on the r.h.s. of the geodesic deviation equation in its Ricci- and Weyl-part, denoted here by $\boldsymbol{B}_{\boldsymbol{u}}$ and $\boldsymbol{C}_{\boldsymbol{u}}$, respectively. For an arbitrary spherically symmetric spacetime the latter is given by (see (D8) and (D36)

$$
\boldsymbol{C}_{\boldsymbol{u}}=\frac{2 E_{\mathrm{W}}}{R^{3}} \boldsymbol{P}_{\boldsymbol{u}}^{\mathcal{B}}-\frac{E_{\mathrm{W}}}{R^{3}} \boldsymbol{P}_{\boldsymbol{u}}^{S^{2}},
$$

where $\boldsymbol{P}_{\boldsymbol{u}}^{\mathcal{B}}$ and $\boldsymbol{P}_{\boldsymbol{u}}^{s^{2}}$ are, respectively, the $\mathcal{B}$ and $S^{2}$ parts of the projector $\boldsymbol{P}_{\boldsymbol{u}}$. Recall that $\boldsymbol{P}_{\boldsymbol{u}}$ projects onto the subspace of the tangential space orthogonal to $\boldsymbol{u}$ (see (125b)). Equation (D38) is exactly the same expression one gets in Newtonian gravity - provided one identifies $E_{\mathrm{w}}$ with the mass of the central object. The spatial endomorphism $\boldsymbol{C}_{\boldsymbol{u}}$ just describes the familiar volumepreserving tidal deformation which produces an expansion in radial direction and a contraction in the orthogonal directions tangential to the $S O(3)$-orbits.

Concerning the Ricci part $\boldsymbol{B}_{\boldsymbol{u}}$, in the case where $\boldsymbol{u}$ is the velocity field of dust ${ }^{23}$, making use of Einstein's equation (see (A12) ) we have:

$$
\boldsymbol{B}_{\boldsymbol{u}}=-\frac{4 \pi}{3} \varrho \boldsymbol{P}_{\boldsymbol{u}} .
$$

This just says that the local effect of matter (here given by dust) is an isotropic contraction.

\section{Spherically symmetric perfect fluids}

We specialize now to a perfect fluid, which is described by a four-velocity vector field $\boldsymbol{u}$, density $\varrho$, and pressure

\footnotetext{
${ }^{23}$ Recall that dust particles moves along geodesics by the Euler equation.
}

$p$. In case of spherical symmetry $\boldsymbol{u}$ is tangent to the basis manifold and the matter energy-momentum tensor (70) decomposes as

$$
\boldsymbol{T}=\varrho \underline{\boldsymbol{u}} \otimes \underline{\boldsymbol{u}}+p\left(\underline{\boldsymbol{u}} \otimes \underline{\boldsymbol{u}}-\boldsymbol{g}_{\mathcal{B}}\right)+p R^{2} \boldsymbol{g}_{S^{2}},
$$

from which one can read off the part tangent to $\mathcal{B}$ :

$$
\boldsymbol{T}_{\mathcal{B}}=\varrho \underline{\boldsymbol{u}} \otimes \underline{\boldsymbol{u}}+p\left(\underline{\boldsymbol{u}} \otimes \underline{\boldsymbol{u}}-\boldsymbol{g}_{\mathcal{B}}\right) .
$$

Usually, the description is to be completed with the specification of an equation of state. We will not assume any equation of state yet, since in some cases (e.g. McVittie spacetime) this happens to be determined by Einstein's equation.

Inserting (D41) in (D35) we get for the Ricci part of the MS energy the simple expression

$$
E_{\mathrm{R}}=\frac{4 \pi}{3} R^{3} \varrho .
$$

Also the expression (D23) for the differential of the MS energy simplifies in case of a perfect fluid. Using (D41) the Kodama current (as one-form) becomes

$$
\underline{\boldsymbol{j}}=(\varrho+p) \boldsymbol{g}(\boldsymbol{k}, \boldsymbol{u}) \underline{\boldsymbol{u}}-p \underline{\boldsymbol{k}} .
$$

It is useful to introduce an adapted orthonormal basis $\{\boldsymbol{u}, \boldsymbol{e}\}$ tangent to the basis manifold, where $\boldsymbol{u}$ is the velocity vector field of the fluid and $\boldsymbol{e}$ is chosen to point in direction of increasing areal radius. Because of our choice of orientation we have $\underline{\boldsymbol{e}}=\star \underline{\boldsymbol{u}}$ (the volume form on $\mathcal{B}$ is simply $\boldsymbol{\mu}_{\mathcal{B}}=\underline{\boldsymbol{u}} \wedge \underline{\boldsymbol{e}}$ ). Using this expression for $\boldsymbol{e}$ and the definition of the Kodama vector field (D20) we have $\boldsymbol{g}(\boldsymbol{k}, \boldsymbol{u})=\langle\underline{\boldsymbol{k}}, \underline{\boldsymbol{u}}\rangle=\langle\star \boldsymbol{d} R, \underline{\boldsymbol{u}}\rangle=-\langle\boldsymbol{d} R, \star \underline{\boldsymbol{u}}\rangle=$ $-\langle\boldsymbol{d} R, \underline{\boldsymbol{e}}\rangle=-\boldsymbol{d} R(\boldsymbol{e})$ and hence, the Hodge star of the Kodama current becomes

$$
\star \underline{\boldsymbol{j}}=-p \boldsymbol{d} R(\boldsymbol{u}) \underline{\boldsymbol{u}}-\varrho \boldsymbol{d} R(\boldsymbol{e}) \underline{\boldsymbol{e}},
$$

which, inserted in (D23), gives the following expression for the differential of the MS energy for a perfect fluid:

$$
\boldsymbol{d} E=-4 \pi R^{2}(p \boldsymbol{d} R(\boldsymbol{u}) \underline{\boldsymbol{u}}+\varrho \boldsymbol{d} R(\boldsymbol{e}) \underline{\boldsymbol{e}}) .
$$

Hence, the variation of the MS energy along $\boldsymbol{u}$ and $\boldsymbol{e}$ is, respectively:

$$
\begin{aligned}
& \boldsymbol{d} E(\boldsymbol{u})=-4 \pi R^{2} p \boldsymbol{d} R(\boldsymbol{u}), \\
& \boldsymbol{d} E(\boldsymbol{e})=+4 \pi R^{2} \varrho \boldsymbol{d} R(\boldsymbol{e}) .
\end{aligned}
$$

These expressions have a good physical interpretation: Since the matter moves along $\boldsymbol{u}$, (D46a) expresses the fact that the energy can only increase (decrease) if the motion along $\boldsymbol{u}$ does (releases) work against (with) the action of the pressure. Equation (D46b) expresses the almost obvious increase (decrease) of gravitational mass with increase (decrease) of volume in the rest system of the matter. We said 'almost' because $4 \pi R^{2} \boldsymbol{d} R(\boldsymbol{e})$ is not quite the increment of proper volume. The difference accounts for the fact that kinetic and gravitational binding 
energy are themselves gravitationally active. To see that this is indeed what (D46b) implies, let $p$ be some point in spacetime and $S_{p}$ the two-sphere of spherical symmetry through $p$. Assume $S_{p}$ to have a regular interior, that is, that $S_{p}$ bounds a 3 -ball $B_{p}$ in the hypersurface $\Sigma$ orthogonal to $\boldsymbol{u}$. Except for the origin of $B_{p}$, we can write $B_{p}=\sigma \times S^{2}$, where $\sigma$ is a spacelike curve in $\mathcal{B}$ orthogonal to $\boldsymbol{u}$, going from the center of symmetry to $\pi(p)$. Using the expression $E=(R / 2)\left(1+(\boldsymbol{d} R(\boldsymbol{u}))^{2}-(\boldsymbol{d} R(\boldsymbol{e}))^{2}\right)$ for the MS energy to eliminate $\boldsymbol{d} R(\boldsymbol{e})$ in (D46b), integrating the latter over $\sigma$, and re-expressing the result as a volume integral, one gets:

$$
E(p)=\int_{B_{p}} \varrho\left(1+(\boldsymbol{d} R(\boldsymbol{u}))^{2}-\frac{2 E}{R}\right)^{1 / 2} \boldsymbol{\mu}_{\Sigma} .
$$

One sees that the MS energy contains the contribution from the proper mass contained in the ball $B_{p}$,

$$
M(p)=\int_{B_{p}} \varrho \boldsymbol{\mu}_{\Sigma}
$$

as well as contributions from the 'kinetic' and 'potential' energy (Havward, 1996; Misner and Sharp, 1964). In a Newtonian approximation, that is for small 'velocity' $\boldsymbol{d} R(\boldsymbol{u})$ and weak field (small $E / R$ ) one can expand the square root in (D47) and gets, in leading order:

$$
E(p) \approx \int_{B_{p}}\left(\varrho+\frac{1}{2} \varrho(\boldsymbol{d} R(\boldsymbol{u}))^{2}-\frac{\varrho M}{R}\right) \boldsymbol{\mu}_{\Sigma} .
$$

In this approximation the MS energy is therefore just the sum of the proper mass and the Newtonian kinetic and potential energies contained in the ball $B_{p}$. This provides a sound justification for the interpretation of the MS energy as the active gravitational energy.

At this point we can compute also the differentials of the two parts (D34) of the MS energy separately. The differential of the Ricci part follows directly from (D42):

$$
\boldsymbol{d} E_{\mathrm{R}}=4 \pi R^{2}\left(\varrho \boldsymbol{d} R+\frac{1}{3} R \boldsymbol{d} \varrho\right)
$$

and the differential of the Weyl part is just the difference of this with (D45):

$$
\boldsymbol{d} E_{\mathrm{W}}=-4 \pi R^{2}\left((\varrho+p) \boldsymbol{d} R(\boldsymbol{u}) \underline{\boldsymbol{u}}+\frac{1}{3} R \boldsymbol{d} \varrho\right) .
$$

Its components in the directions $\boldsymbol{u}$ and $\boldsymbol{e}$ are then

$$
\begin{aligned}
& \boldsymbol{d} E_{\mathrm{W}}(\boldsymbol{u})=-4 \pi R^{2}(\varrho+p) \boldsymbol{d} R(\boldsymbol{u})-\frac{4 \pi}{3} R^{3} \boldsymbol{d} \varrho(\boldsymbol{u}), \\
& \boldsymbol{d} E_{\mathrm{W}}(\boldsymbol{e})=-\frac{4 \pi}{3} R^{3} \boldsymbol{d} \varrho(\boldsymbol{e}) .
\end{aligned}
$$

It is now instructive to express the variation along $\boldsymbol{u}$ of the Ricci and Weyl parts of the MS energy in terms of the kinematical properties of the fluid velocity $\boldsymbol{u}$. Recall that, because of spherical symmetry, the rotation tensor vanishes identically and the shear tensor has only one independent component. The kinematical quantities reduces thus to two scalars: the expansion

$$
\theta:=\operatorname{div} u
$$

and the shear scalar

$$
\sigma:=\frac{\boldsymbol{d} R(\boldsymbol{u})}{R}-\frac{1}{3} \theta .
$$

The shear tensor is then given by the trace-free endomorphism $\boldsymbol{\sigma}=\sigma\left(\boldsymbol{Q}_{S^{2}}-2 \boldsymbol{Q}_{\boldsymbol{e}}\right)$, where $\boldsymbol{Q}_{S^{2}}$ and $\boldsymbol{Q}_{\boldsymbol{e}}$ denote, respectively, the projections onto the two-dimensional subspace of $T(\mathcal{M})$ tangential to the two-sphere and onto the one-dimensional space parallel to $\boldsymbol{e}$ (for the latter see (C1a)). We recall that the divergence-freeness of the energy-momentum tensor (D40) is equivalent to

$$
\begin{aligned}
& (\varrho+p) \theta=-\boldsymbol{d} \varrho(\boldsymbol{u}) \\
& (\varrho+p) b=-\boldsymbol{d} p(\boldsymbol{e}),
\end{aligned}
$$

where $b:=-\boldsymbol{g}\left(\nabla_{\boldsymbol{u}} \boldsymbol{u}, \boldsymbol{e}\right)$ is the acceleration (scalar) of $\boldsymbol{u}$ in positive radial direction (the minus sign in the latter formula is because the metric is negative definite in spatial directions).

Now, using (D55a) and (D54) we get:

$$
\begin{aligned}
& \boldsymbol{d} E_{\mathrm{R}}(\boldsymbol{u})=\frac{4 \pi}{3} R^{3}(3 \varrho \sigma-p \theta) \\
& \boldsymbol{d} E_{\mathrm{W}}(\boldsymbol{u})=-\frac{4 \pi}{3} R^{3}(\varrho+p) 3 \sigma
\end{aligned}
$$

With the equations just derived we can now say when the MS energy, and its Ricci and Weyl parts, are temporally or spatially constant. Here, by temporally (spatially) constant we mean that the variation in direction of $\boldsymbol{u}(\boldsymbol{e})$ vanishes. We collect the results in the following

Theorem 4. Consider a spherically symmetric fluid with $\varrho+p \neq 0$ and restrict to the region where $d R$ is spacelike. Then for the MS energy $E$ and its Ricci and Weyl parts $E_{R}$ and $E_{W}$ the following statements hold true:

(i) $E$ is temporally constant iff $p=0$ or $\boldsymbol{d} R(\boldsymbol{u})=0$;

(ii) $E$ is spatially constant iff $\varrho=0$;

(iii) $E_{W}$ is temporally constant iff $\sigma=0$;

(iv) $E_{W}$ is spatially constant iff $\varrho$ is spatially constant;

(v) $E_{R}$ is temporally (spatially) constant iff $R^{3} \varrho$ is temporally (spatially) constant.

The proof is a straightforward application of the formulae just derived above. Note that the assumption $\varrho+p \neq$ 0 is needed only for (iii). The assumption that $\boldsymbol{d} R$ is spacelike is needed only for (ii): If $\boldsymbol{d} R$ is spacelike, then for any spacelike spherically symmetric vector $\boldsymbol{e}$ (hence tangent to the basis manifold $\mathcal{B})$ it holds $\boldsymbol{d} R(\boldsymbol{e}) \neq 0$, since in a two-dimensional Lorentzian manifold any two spacelike vectors are linearly dependent.

\section{References}

Adkins, G. S., J. McDonnell, and R. N. Fell, 2007, "Cosmological perturbations on local systems," Physical Review D 75(6), 064011 (pages 9). 
Anderson, J. D., P. A. Laing, E. L. Lau, A. S. Liu, M. M. Nieto, and S. G. Turyshev, 1998, "Indication, from Pioneer 10/11, Galileo, and Ulysses Data, of an Apparent Anomalous, Weak, Long-Range Acceleration," Physical Review Letters 81, 2858-2861.

Anderson, J. D., P. A. Laing, E. L. Lau, A. S. Liu, M. M. Nieto, and S. G. Turyshev, 2002, "Study of the anomalous acceleration of Pioneer 10 and 11," Physical Review D 65(8), 082004 (pages 50).

Balbinot, R., R. Bergamini, and A. Comastri, 1988, "Solution of the Einstein-Strauss problem with a $\Lambda$ term," Physical Review D 38, 2415-2418.

Barnes, L. A., M. J. Francis, J. B. James, and G. F. Lewis, 2006, "Joining the Hubble Flow: Implications for Expanding Space," Monthly Notices of the Royal Astronomical Society 373(1), 382-390.

Bini, D., P. Carini, and R. T. Jantzen, 1995, "Relative Observer Kinematics in General Relativity," Classical and Quantum Gravity 12, 2549-2563.

Bolen, B., L. Bombelli, and R. Puzio, 2001, "Expansioninduced contribution to the precession of binary orbits," Classical and Quantum Gravity 18, 1173-1178.

Bolós, V. J., 2007, "Intrinsic Definitions of "Relative Velocity" in General Relativity," Communications in Mathematical Physics 273(1), 217-236.

Bona, C., and J. Stela, 1987, "SWwiss cheese" models with pressure," Physical Review D 36(10), 2915-2918.

Bonnor, W. B., 1999, "Size of a hydrogen atom in the expanding universe," Classical and Quantum Gravity 16, 13131321.

Bonnor, W. B., 2000, "A generalization of the Einstein-Straus vacuole," Classical and Quantum Gravity 17, 2739-2748.

Buchert, T., 2000, "On Average Properties of Inhomogeneous Fluids in General Relativity: Dust Cosmologies," General Relativity and Gravitation 32(1), 105-125.

Buchert, T., 2008, "Dark Energy from Structure - A Status Report," General Relativity and Gravitation 40, 467-527.

Buchert, T., and M. Carfora, 2003, "Cosmological Parameters Are Dressed," Physical Review Letters 90(3), 031101 (pages 4).

Buchert, T., and A. Dominguez, 2005, "Adhesive Gravitational Clustering," Astronomy and Astrophysics 438, 443460.

Burnett, G. A., 1991, "Incompleteness theorems for the spherically symmetric spacetimes," Physical Review D 43, 11431149.

Cahill, M. E., and G. C. McVittie, 1970a, "Spherical Symmetry and Mass-Energy in General Relativity. I. General Theory," Journal of Mathematical Physics 11(4), 1382-1391.

Cahill, M. E., and G. C. McVittie, 1970b, "Spherical Symmetry and Mass-Energy in General Relativity. II. Particular Cases," Journal of Mathematical Physics 11(4), 1392-1401.

Carrera, M., 2009, Ph.D. thesis, University of Freiburg (Germany).

Carrera, M., and D. Giulini, 2005, "On the influence of the global cosmological expansion on the local dynamics in the Solar System,", European Space Agency, the Advanced Concepts Team, Ariadna Final Report 04-1302, eprint arXiv:gr-qc/0602098.

Carrera, M., and D. Giulini, 2006, "On Doppler tracking in cosmological spacetimes," Classical and Quantum Gravity 23, 7483-7492.

Carrera, M., and D. Giulini, 2009, "On the generalization of McVittie's model for an inhomogeneity in a cosmological spacetime," eprint arXiv:0908.3101.

Célérier, M.-N., 2000, "Do we really see a cosmological constant in the supernovae data?," Astronomy and Astrophysics 353, 63-71.

Cooperstock, F. I., V. Faraoni, and D. N. Vollick, 1998, "The Influence of the Cosmological Expansion on Local Systems," Astrophysical Journal 503, 61-66.

Cox, D. P. G., 2007, "How far is "infinity'?," General Relativity and Gravitation 39(2), 87-104.

Darmois, G., 1927, "Les équations de la gravité einsteinienne," Mémorial des sciences mathématiques XXV, 1-47.

Dicke, R. H., and P. J. E. Peebles, 1964, "Evolution of the Solar System and the Expansion of the Universe," Physical Review Letters 12, 435-437.

Dominguez, A., and J. Gaite, 2001, "Influence of the Cosmological Expansion on Small Systems," Europhysics Letters 55(4), 458-464.

Einstein, A., and E. G. Straus, 1945, "The Influence of the Expansion of Space on the Gravitation Fields Surrounding the Individual Stars," Reviews of Modern Physics 17, 120124.

Einstein, A., and E. G. Straus, 1946, "Corrections and Additional Remarks to our Paper: The Influence of the Expansion of Space on the Gravitation Fields Surrounding the Individual Stars," Reviews of Modern Physics 18, 148-149.

Eisenstaedt, J., 1977, "Density constraint on local inhomogeneities of a Robertson-Walker cosmological universe," Physical Review D 16, 927-928.

Fahr, H. J., and M. Siewert, 2008, "Imprints from the Global Cosmological Expansion on the Local Spacetime Dynamics," Naturwissenschaften 95(5), 413-425.

Faraoni, V., and A. Jacques, 2007, "Cosmological Expansion and Local Physics," Physical Review D 76, 063510 (pages 16).

Ferraris, M., M. Francaviglia, and A. Spallicci, 1996, "Associated radius, energy and pressure of McVittie's metric, in its astrophysical application," Nuovo Cimento B111, 1031-1036.

Fouqué, P., J. M. Solanes, T. Sanchis, and C. Balkowski, 2001, "Structure, mass and distance of the Virgo cluster from a Tolman-Bondi model," Astronomy and Astrophysics 375, 770-780.

Gao, C. J., and S. N. Zhang, 2004, "Reissner-Nordström metric in the Friedman-Robertson-Walker universe," Physics Letters B 595, 28-35.

Gautreau, R., 1984, "Imbedding a Schwarzschild mass into cosmology," Physical Review D 29, 198-206.

Geyer, K. H., 1980, "Geometrie der Raum-Zeit der Maßbestimmung von Kottler, Weyl und Trefftz," Astronomische Nachrichten 301(3), 135-149.

Giulini, D., 1998, "On the Construction of Time-Symmetric Black Hole Initial Data," LNP Vol. 514: Black Holes: Theory and Observation 514, 224-243.

Hackmann, E., and C. Lämmerzahl, 2008a, "Complete analytic solution of the geodesic equation in Schwarzschild(anti-)de Sitter spacetimes," Physical Review Letters 100, 171101 (pages 4).

Hackmann, E., and C. Lämmerzahl, 2008b, "Geodesic Equation and Theta-Divisor," AIP Conference Proceedings 977(1), 116-133.

Hackmann, E., and C. Lämmerzahl, 2008c, "Geodesic equation in Schwarzschild-(anti-)de Sitter space-times: Analytical solutions and applications," Physical Review D 78(2), 024035 (pages 22). 
Hartl, M. G., 2006, Untersuchungen über den Einfluss der kosmologischen Expansion auf die Dynamik lokaler Systeme, Diploma thesis, University of Freiburg, Germany.

Hawking, S. W., 1968, "Gravitational Radiation in an Expanding Universe," Journal of Mathematical Physics 9(4), 598-604.

Hayward, S. A., 1996, "Gravitational energy in spherical symmetry," Physical Review D 53, 1938-1949.

Hayward, S. A., 1998, "Inequalities Relating Area, Energy, Surface Gravity, and Charge of Black Holes," Physical Review Letters 81, 4557-4559.

Hernandez, J., Walter C., and C. W. Misner, 1966, "Observer Time as a Coordinate in Relativistic Spherical Hydrodynamics," Astrophysical Journal 143, 452-464.

Hubble, E., 1929, "A Relation Between Distance and Radial Velocity Among Extra-Galactic Nebulae," Proceedings of the National Academy of Sciences of the United States of America 15(3), 168-173.

Israel, W., 1966, "Singular Hypersurfaces and Thin Shells in General Relativity," Nuovo Cimento 44B(1), 1-14; Errata ibid 48B(2), 463.

Kagramanova, V., J. Kunz, and C. Lämmerzahl, 2006, "Solar system effects in Schwarzschild-de Sitter space-time," Physics Letters B 634, 465-470.

Klioner, S. A., and M. H. Soffel, 2005, "Refining the Relativistic Model for Gaia: Cosmological Effects in the BCRS," in The Three-Dimensional Universe with Gaia, edited by C. Turon, K. S. O'Flaherty, and M. A. C. Perryman (ESA), volume 576 of ESA Special Publication, 305-308, proceedings of the Symposium "The Three-Dimensional Universe with Gaia", 4-7 October 2004, Observatoire de Paris-Meudon, France, eprint arXiv:astro-ph/0411363.

Kodama, H., 1980, "Conserved Energy Flux for the Spherically Symmetric System and the Backreaction Problem in the Black Hole Evaporation," Progress of Theoretical Physics 63(4), 1217-1228.

Komatsu, E., et al., 2009, "Five-Year Wilkinson Microwave Anisotropy Probe (WMAP) Observations: Cosmological Interpretation," Astrophysical Journal, Supplement Series 180, 330-376.

Kottler, F., 1918, "Über die physikalischen Grundlagen der Einsteinschen Gravitationstheorie," Annalen der Physik 56(14), 401-462.

Krasiński, A., 1998, Inhomogeneous Cosmological Models, Cambridge Monographs on Mathematical Physics (Cambridge University Press, Cambridge).

Krasinsky, G. A., and V. A. Brumberg, 2004, "Secular increase of astronomical unit from analysis of the major planet motions, and its interpretation," Celestial Mechanics and Dynamical Astronomy 90, 267-288.

Lämmerzahl, C., O. Preuss, and H. Dittus, 2006, "Is the physics within the Solar system really understood?," eprint arXiv:gr-qc/0604052.

Lanczos, K., 1924, "Flächenhafte Verteilung der Materie in der Einsteinschen Gravitationstheorie," Annalen der Physik 379(14), 518-540.

Lemaître, G., 1933, "L'Univers en expansion," Annales de la Société Scientifique de Bruxelles, Series A, Sciences Mathématiques, Astronomiques et Physiques 53, 51-85.

Lemaître, G., 1997, "The expanding universe," General Relativity and Gravitation 29, 641-680 traduction of the original article (Lemaître, 1933).

Markwardt, C. B., 2002, "Independent Confirmation of the Pioneer 10 Anomalous Acceleration," eprint arXiv:gr- qc/0208046.

McClure, M. L., 2006, Cosmological Black Holes as models of cosmological inhomogeneities, Ph.D. thesis, University of Toronto.

McVittie, G. C., 1933, "The mass-particle in an expanding universe," Monthly Notices of the Royal Astronomical Society 93, 325-339.

Mena, F. C., R. Tavakol, and R. Vera, 2002, "Generalization of the Einstein-Straus model to anisotropic settings," Physical Review D 66(4), 044004 (pages 13).

Mena, F. C., R. Tavakol, and R. Vera, 2003, "On Modifications of the Einstein-Straus Model to Anisotropic Settings," LNP Vol. 617: Current Trends in Relativistic Astrophysics 617, 343-347.

Mena, F. C., R. Tavakol, and R. Vera, 2005, "Generalisations of the Einstein-Straus model to cylindrically symmetric settings," in The Tenth Marcel Grossmann Meeting. On recent developments in theoretical and experimental general relativity, gravitation and relativistic field theories, edited by M. Novello, S. Perez Bergliaffa, and R. Ruffini (World Scientific, Singapore), 1749-1751, eprint arXiv:gr-qc/0405043.

Misner, C. W., and D. H. Sharp, 1964, "Relativistic Equations for Adiabatic, Spherically Symmetric Gravitational Collapse," Physical Review 136, 571-576.

Misner, C. W., K. S. Thorne, and J. A. Wheeler, 1973, Gravitation (W.H. Freeman and Company, New York).

Nieto, M. M., and S. G. Turyshev, 2004, "Finding the origin of the Pioneer anomaly," Classical and Quantum Gravity 21(17), 4005-4023.

Nieto, M. M., S. G. Turyshev, and J. D. Anderson, 2005, "Directly measured limit on the interplanetary matter density from Pioneer 10 and 11," Physics Letters B 613, 11-19.

Noerdlinger, P. D., and V. Petrosian, 1971, "The Effect of Cosmological Expansion on Self-Gravitating Ensembles of Particles," Astrophysical Journal 168, 1-9.

Nolan, B. C., 1993, "Sources for McVittie's Mass Particle in an Expanding Universe," Journal of Mathematical Physics 34(1), 178-185.

Nolan, B. C., 1998, "A point mass in an isotropic universe: Existence, uniqueness, and basic properties," Physical Review D 58(6), 064006 (pages 10).

Nolan, B. C., 1999a, "A point mass in an isotropic universe: II. Global properties," Classical and Quantum Gravity 16, 1227-1254.

Nolan, B. C., 1999b, "A point mass in an isotropic universe: III. The region $R \leq 2 m$," Classical and Quantum Gravity 16, 3183-3191.

Nottale, L., 2003, "The Pioneer anomalous acceleration: a measurement of the cosmological constant at the scale of the solar system," eprint arXiv:gr-qc/0307042.

O'Neill, B., 1983, Semi-Riemannian Geometry with Applications to Relativity (Academic Press, Orlando).

Pachner, J., 1963, "Mach's Principle in Classical and Relativistic Physics," Physical Review 132(4), 1837-1842.

Pachner, J., 1964, "Nonconservation of Energy During Cosmic Evolution," Physical Review Letters 12(4), 117-118.

Palle, D., 2005, "On the anomalous acceleration in the solar system," Acta Physica Slovaca 55(2), 237-240.

Patel, L. K., and H. B. Trivedi, 1982, "Kerr-Newman metric in cosmological background," Journal of Astrophysics and Astronomy 3, 63-67.

Price, R. H., 2005, "In an expanding universe, what doesn't expand?," eprint arXiv:gr-qc/0508052.

Rajesh Nayak, K., M. A. MacCallum, and C. V. Vishvesh- 
wara, 2001, "Black holes in nonflat backgrounds: The Schwarzschild black hole in the Einstein universe," Physical Review D 63(2), 024020 (pages 5).

Ramachandra, B. S., K. R. Nayak, and C. V. Vishveshwara, 2003, "Kerr Black Hole in the Background of the Einstein Universe," General Relativity and Gravitation 35, 19772005.

Ramachandra, B. S., and C. V. Vishveshwara, 2002, "Schwarzschild black hole in the background of the Einstein universe: some physical effects," Classical and Quantum Gravity 19, 127-141.

Ranada, A. F., 2005, "The Pioneer anomaly as acceleration of the clocks," Foundations of Physics 34, 1955-1971.

Räsänen, S., 2006, "Accelerated expansion from structure formation," Journal of Cosmology and Astroparticle Physics 11, 003 (pages 40).

Robertson, H. P., 1928, "On Relativistic Cosmology," Philosophical Magazine 5, 835-848.

Rosales, J., and J. Sanchez-Gomez, 1998, "The "Pioneer effect" as a manifestation of the cosmic expansion in the solar system," eprint arXiv:gr-qc/99810085.

Rosales, J. L., 2002, "The Pioneer's acceleration anomaly and Hubble's constant," eprint arXiv:gr-qc/0212019.

Schücking, E., 1954, "Das Schwarzschildsche Linienelement und die Expansion des Weltalls," Zeitschrift für Physik 137, 595-603.

Senovilla, J. M. M., and R. Vera, 1997, "Impossibility of the Cylindrically Symmetric Einstein-Straus Model," Physical Review Letters 78, 2284-2287.

Sereno, M., and P. Jetzer, 2007, "Evolution of Gravitational Orbits in the Expanding Universe," Physical Review D 75(6), 064031 (pages 8).

Standish, E. M., 2004, "The Astronomical Unit now," in Transits of Venus: New Views of the Solar System and Galaxy, Proceedings of the IAU Colloquium No. 196, 2004, edited by D. W. Kurtz (Cambridge University Press, Cambridge), 163-179.

Straumann, N., 2004, General Relativity with Applications to Astrophysics, Texts and Monographs in Physics (SpringerVerlag, Berlin).
Sultana, J., and C. C. Dyer, 2005, "Cosmological black holes: A black hole in the Einstein-de Sitter universe," General Relativity and Gravitation 37(8), 1349-1370.

Sussman, R. A., 1988, "On spherically symmetric shearfree perfect fluid configurations (neutral and charged). III. Global view," Journal of Mathematical Physics 29(5), 1177-1211.

Szabados, L. B., 2004, "Quasi-Local Energy-Momentum and Angular Momentum in GR: A Review Article," Living Reviews in Relativity $\mathbf{7}(4)$, 1-135, URL http://www . livingreviews.org/lrr-2004-4.

Turyshev, S. G., M. M. Nieto, and J. D. Anderson, 2005a, "A route to understanding of the Pioneer anomaly," eprint arXiv:gr-qc/0503021.

Turyshev, S. G., M. M. Nieto, and J. D. Anderson, 2005b, "Study of the Pioneer anomaly: A problem set," American Journal of Physics 73(11), 1033-1044.

Vaidya, P. C., 1977, "The Kerr metric in cosmological background," Pramana 8(6), 512-517.

Vaidya, P. C., 1984, "Kerr metric in the deSitter background.," Pramana 22, 151-158.

van den Bergh, N., and P. Wils, 1984, "Imbedding a Schwarzschild mass into cosmology," Physical Review D 29, 3002-3003.

Vishveshwara, C. V., 2000, "Black Holes in cosmological backgrounds," in The Universe: Visions and Perspectives, edited by A. K. K. Naresh Dadhich (Kluwer Academic Publisher), 309-318.

Wiltshire, D. L., 2007, "Exact Solution to the Averaging Problem in Cosmology," Physical Review Letters 99(25), 251101 (pages 4).

Wiltshire, D. L., 2008, "Dark energy without dark energy," in Dark Matter in Astroparticle and Particle Physics: Proceedings of the 6th International Heidelberg Conference (World Scientific, Singapore), 565-596, eprint arXiv:0712.3984.

Zannias, T., 1990, "Spacetimes admitting a three-parameter group of isometries and quasilocal gravitational mass," Physical Review D 41(10), 3252-3254. 